\title{
CY 1995 Radiation Dose Reconciliation Report and. Resulting CY 1996 Dose Estimate for the 324 Nuclear Facility
}
S. D. Landsman
R. E. Thornhill
C. A. Peterson

April 1996

Prepared for the U.S. Department of Energy under Contract DE-AC06-76RLO 1830

Pacific Northwest National Laboratory Operated for the U.S. Department of Energy by Battelle 
This report was prepared as an account of work sponsored by an agency of the United. States Government. Neither the United States Government nor any agency thereof, nor Battelle Memorial Institute, nor any of their employees, makes any warranty, express or implied, or assumes any legal liability or responsibility for the accuracy, completeness, or usefulness of any information, apparatus, product, or process disclosed, or represents that its use would not infringe privately owned rights. Reference herein to any specific commercial product, process, or service by trade name, trademark, manufacturer, or otherwise does not necessarily constitute or imply its endorsement, recommendation, or favoring by the United States Government or any agency thereof, or Battelle Memorial Institute. The views and opinions of authors expressed herein do not necessarily state or reflect those of the United States Government or any agency thereof.

\section{PAĆIIIC NORTHWEST NATIONAL LABORATORY \\ operated by \\ BATTELLE \\ for the \\ UNITED STATES DEPARTMENT OF ENERGY \\ under Contract DE-ACO6-76RLO 1830}

Printed in the United States of America

Available to DOE and DOE contractors from the

Office of Scientific and Technical Information, P.O. Box 62, Oak Ridge,

TN 37831;

prices available from (615) 576-8401.

Available to the public from the National Technical Information Service, U.S. Department of Commerce, 5285 Port Royal Rd., Springfield, VA 22161

This document was printed on recycled paper. 


\section{CY 1995 Radiation Dose Reconciliation Report and Resulting CY 1996 Dose Estimate for the 324 Nuclear Facility}

S.D. Landsman

R.E. Thornhill

C.A. Peterson

April 1996

Prepared for the U.S. Department of Energy under Contract DE-AC06-76RLO 1830

Pacific Northwest National Laboratory

Richland, Washington 99352 



\section{Summary}

Dose estimates for 324 Facility radiological work planned through the year 2000 were prepared in CY 1995. Historical radiation dose records of all known radiation work for the various work groups were used as the basis for the estimates. In this report, the estimate for CY 1995 is reconciled (by month) with actual doses received. Results of the reconciliation were used to revise estimates of worker dose for CY 1996. The resulting dose estimate for the facility is also included in the report.

Support for two major programs (B-Cell Cleanout and Surveillance and Maintenance) accounts for most of the exposure received by workers in the facility. The majority of the exposure received by workers comes from work in the Radiochemical Engineering Complex airlock. In spite of unforeseen schedule and work scope changes during CY 1995, dose estimates were generally quite close to actual exposures received. A number of "as low as reasonably achievable" (ALARA) measures were taken throughout the year that enabled the facility workers to reduce cumulative dose received. Actual doses received were generally at or below the ALARA facility goal, which was to reduce worker radiation exposure to $80 \%$ of the CY estimated dose. Overall, exposure reduction due to ALARA measures was 20.6 Man-Rem. This was a 28\% reduction from the CY 1995 estimate.

Data gathered during CY 1995 were used to generate baseline estimates for various tasks performed in the facility. These baseline estimates were used to compile the CY 1996 dose estimate of 45.4 Man-Rem. Although the new baseline estimates are not as conservative as those used to prepare the CY 1995 dose estimate report, the facility goal for CY 1996 is to reduce worker dose by 20\%, to $36.3 \mathrm{Man}-\mathrm{Rem}$. 


\section{Contents}

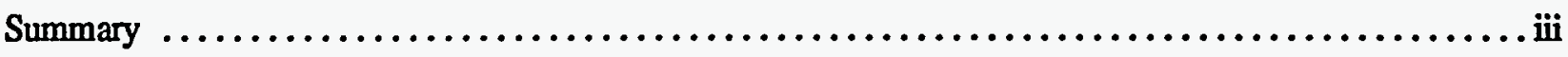

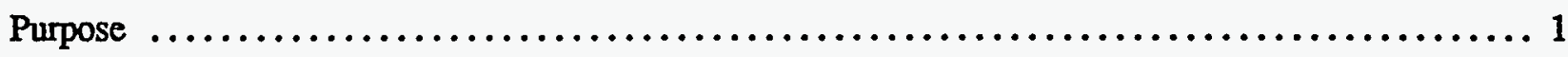

Discussion of CY 1995 Dose Estimate ........................................ 1

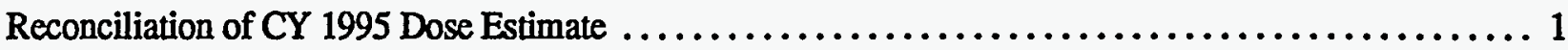

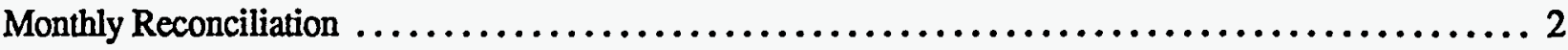

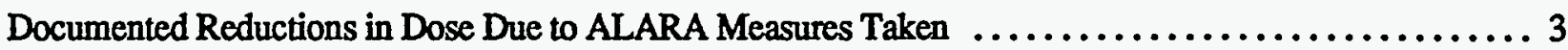

Elimination or Postponement of Tasks $\ldots \ldots \ldots \ldots \ldots \ldots \ldots \ldots \ldots \ldots \ldots \ldots \ldots \ldots, \ldots \ldots \ldots$

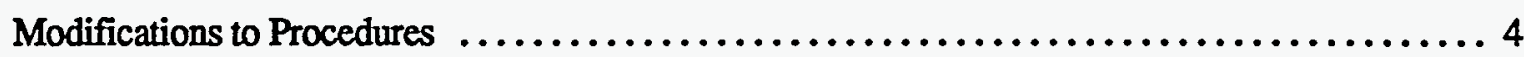

Reduction of Dose Through Decontamination or Other Means $\ldots \ldots \ldots \ldots \ldots \ldots \ldots \ldots \ldots 6$

Manipulator Changes and Repair Activities $\ldots \ldots \ldots \ldots \ldots \ldots \ldots \ldots \ldots \ldots, 6$

C-Cell Decontamination .................................... 9

Total Documented Dose Savings for CY $1995 \ldots \ldots \ldots \ldots \ldots \ldots \ldots \ldots \ldots \ldots \ldots \ldots \ldots \ldots \ldots \ldots$. 9

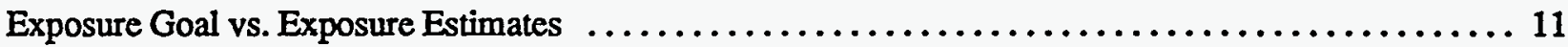

Basis of Estimates for CY 1996 Facility Dose Estimate.$\ldots \ldots \ldots \ldots \ldots \ldots \ldots \ldots \ldots \ldots \ldots \ldots \ldots$

Appendix A: Comparison by Month of Estimates vs. Actuals with Jobs Identified . . . . . . . . . . A.1

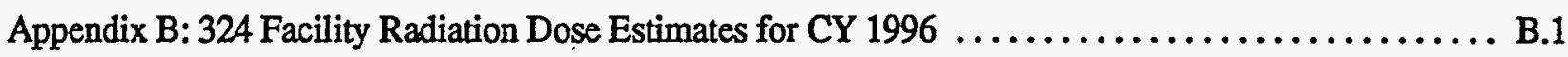

\section{Tables}

1. Comparison by Month of Estimated Dose and Actual Dose Received $\ldots \ldots \ldots \ldots \ldots \ldots \ldots \ldots, 3$

2. Dose from Low-Level Waste Hauls .................................... 5

3. Dose from Manipulator Changes ........................................ 7

4. Dose from Manipulator Repairs .......................................... 8

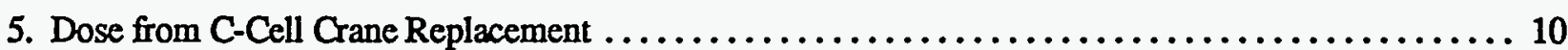

6. Baseline Job Estimates . . . . . . . . . . . . . . . . . . . . . . . . . . 12 



\section{Purpose}

In this report, calendar year (CY) 1995 dose estimates for 324 facility radiological work are reconciled against actual doses received for completion of activities. The reconciliation data are then used to revise estimates of worker dose for CY 1996. The completion date for one of the major facility programs (the B-Cell Cleanout project) has been accelerated, significantly affecting work activities scheduled for the out years of the project. Resulting schedule changes negate the forecast facility dose estimates (see PNL-10739, 324 Building Life Cycle Dose Estimates for Planned Work, S.D. Landsman). At the time of this report, the work plan and schedule for the project had not been finalized for the out years $(1997,1998)$. An addendum to this report, including new worker radiation exposure forecasts for the out years, will be made available when the new work scope for the life of the B-Cell Cleanout project is finalized.

\section{Discussion of CY 1995 Dose Estimate}

Radiological exposure received by workers in the 324 Building is primarily the result of support for two major programs: B-Cell Cleanout and facility Surveillance and Maintenance. The majority of exposure $(-90 \%)$ in the facility is received by personnel working in the Radiochemical Engineering Complex (REC) airlock.

The CY 1995 dose estimate was prepared based on existing schedules for work in the facility. To facilitate tracking actual worker exposures, the estimate was prepared using Prima Vera, software designed to prepare and track program budgets (for further information, see PNL-10739, 324 Building Life Cycle Dose Estimates for Planned Work, S.D. Landsman). The dose estimate details resources needed to complete separate work activities and sorts them by date. The prepared estimate included all known radiation work for the various work groups in the facility: Hot Cell Operations, Radiological Control, Pacific Northwest National Laboratory (PNNL) $)^{(n)}$ Crafts, and Kaiser Crafts. Historical information was used to determine the personnel resources required for each activity listed in the estimate and the amount of time spent in radiation dose fields by those personnel. To facilitate work planning, the Prima Vera software was also used to sort the dose estimate by worker craft and activity. This provided a ready guide for management to consult when determining the need for worker-dose-limit upgrades, or to determine staffing requirements for a particular work activity.

\section{Reconciliation of CY 1995 Dose Estimate}

Tasks scheduled for a given month were not necessarily completed on time, due to equipment failures, schedule changes, delays, changes in work priority, and the addition of new tasks. For example, B-Cell 6-ton and D-Cell 5-ton crane breakdowns postponed scheduled activities because the cranes were not available to perform those tasks. The high priority given to the repair work also did not allow completion of other scheduled work.

(a) Pacific Northwest National Laboratory (previously Pacific Northwest Laboratory) is operated for the U.S. Department of Energy by Battelle under Contract DE-ACO6-76RLO 1830. 
Additionally, work scope changes, such as the unexpected resumption of the cesium legacy program work in the Shielded Materials Facility (SMF), resulted in the addition of activities to support the program. These activities were not included in the original estimate. This change in scope also resulted in several activities scheduled for the SMF complex being postponed to CY 1996.

Analysis of actual exposure received vs. estimated exposure for any given task revealed that in some cases (manipulator changes, C-Cell decontamination) dose estimates were much higher than actuals. Both the frequency of performing a task and the dose estimate for specific tasks were analyzed, based on actual work completed in CY 1995. As a result of this study, dose estimates and task frequencies were adjusted in the CY 1996 dose estimate.

Despite these challenges, however, dose estimates for the CY were generally quite close to actual exposures received by workers in the facility. The majority of tasks involving radiation exposure occur in the REC airlock. Analysis of actual dose taken in the airlock indicates that the amount of time spent in the airlock is more important than the nature of the task being performed. This is true because penetrating dose received in the airlock is consistent at $100 \mathrm{mR}$ to $150 \mathrm{mR}$ per hour of work. Due to the physical nature of work performed in the airlock and the necessity for personal protective equipment (PPE) that creates heat stress and efficiency problems, stay times in the airlock are governed by physical limitations. This creates conditions where airlock entries are of consistent duration.

Since only one task can be performed in the airlock at a time, dose received while performing unexpected work (not included in the CY 1995 dose estimate) was balanced by the dose savings from the task included in the dose estimate that was not performed. Although this has been presumed in the past, substantiating documentation was incomplete. In CY 1995, complete dose documentation of all entries to the airlock was gathered and maintained on a consistent basis for the first time.

In general, actual doses received by personnel for specific tasks or evolutions were at or below "as low as reasonably achievable" (ALARA) goal estimates, which were established as $80 \%$ of the estimated dose for the activity. ${ }^{(\bullet)}$ Later in this report, ALARA measures initiated in the facility that contributed to the dose savings are discussed.

\section{Monthly Reconciliation}

Table 1 summarizes doses received, by month, for all radiation area work during CY 1995. The ratio of actual dose to estimated dose is a measure of the overall accuracy of the dose estimates. To provide more accurate comparison of actual dose to estimated dose, jobs that were completed but not included in the original CY 1995 dose estimate report were assigned dose estimates "after the fact." These estimates were prepared in the same manner as estimates for jobs that were included in the CY 1995 dose-estimate report. Estimated dose is presented in three different formats: 1) CY estimated dose for those jobs that were included in the CY 1995 dose-estimate report, 2) actual estimated dose, representing the estimate for work completed in the month that was included in the CY dose estimate, and 3) adjusted estimated dose, representing the estimate for all work performed during the month, including unexpected tasks. Actual dose shown is for all jobs completed in the month.

(a) This goal for CY 1995 was set in PNL-10739, 324 Building Life Cycle Dose Estimates for Planned Work. It was set to be a challenging ALARA goal for reduction of worker radiation exposures. 
Table 1. Comparison by Month of Estimated Dose and Actual Dose Received (Dose information is in mRem)

\begin{tabular}{|c|c|c|c|c|c|}
\hline Month & $\begin{array}{c}\text { CY } \\
\text { Estimated } \\
\text { Dose } \\
\end{array}$ & $\begin{array}{c}\text { Actual } \\
\text { Estimated } \\
\text { Dose } \\
\end{array}$ & $\begin{array}{l}\text { Adjusted } \\
\text { Estimated } \\
\text { Dose" } \\
\end{array}$ & Actual Dose & Actual/Estimate $e^{(\phi)}$ \\
\hline January & 3731 & 2348 & 2736 & 1793 & 0.66 \\
\hline February & 7137 & 5852 & 7142 & 3909. & 0.55 \\
\hline March & 6922 & 2416 & 2796 & 1946 & 0.70 \\
\hline April & 12801 & 2322 & 2322 & 2025 & 0.87 \\
\hline May & 7584 & 871 & 1946 & 1633 & 0.84 \\
\hline June & 18904 & 8397 & 8397 & 6435 & 0.77 \\
\hline July & 5881 & 6271 & 6271 & 6966 & 1.11 \\
\hline August & 6346 & 7058 & 7373 & 7851 & 1.06 \\
\hline September & 5132 & 5168 & 5168 & 3551 & 0.69 \\
\hline October & 1815 & 4908 & 4908 & 6350 & 1.29 \\
\hline November & 2161 & 799 & 1019 & 1353 & 1.33 \\
\hline December & 1230 & 3488 & 3608 & 3803 & 1.05 \\
\hline Total & 79644 & 49898 & 53686 & 47615 & 0.89 \\
\hline
\end{tabular}

(a) Estimated dose for the month from the CY 1995 dose estimate

(b) Cumulative estimates for jobs that actually occurred during the month and that had been included in the CY 1995 dose estimate

(C) Cumulative estimates for all jobs that actually occurred during the month, whether they were included in the original CY 1995 dose estimate or not. This column is necessary to compare actual dose received to the estimated dose.

(d) The estimated dose used in this calculation is the adjusted estimated dose.

\section{Documented Reductions in Dose Due to ALARA Measures Taken}

Some of the tasks performed in CY 1995 had dose reductions due to ALARA measures taken before or during the task evolution. These ALARA measures fell into several categories: 1) elimination or postponement of a task, 2) procedural changes that reduced exposure, and 3) reduction of dose rates through decontamination or other means.

\section{Elimination or Postponement of Tasks}

Several tasks originally scheduled for completion in CY 1995 were eliminated from the schedule or postponed for ALARA reasons. The major task eliminated from the schedule was installation of cell cleaning systems by Kaiser Engineers, Hanford (KEH). In this task, several shield plugs containing service connections between the hot cells and the galleries to support the use of the Ultra High Pressure Spray Decontamination system were removed and replaced. It was determined that this work could be eliminated if the system were installed in the airlock because all of the hot cells could be accessed from the airlock, eliminating the need to provide individual services to the cells. Elimination of this task from the schedule 
resulted in a savings of 3.1 man-Rem. This savings largely contributes to the difference between CY estimated and actual doses for January and February.

Installation of the 3.5-ton crane in B-Cell was postponed for ALARA reasons. It was determined that the crane was not vital to operations in CY 1995, and installation could be postponed until the crane was needed to remove the $1 \mathrm{~A}$ and $1 \mathrm{~B}$ racks. Installation at a later time allows completion of other tasks, such as low-level-waste characterization and shipping, resulting in increased dose rates in the airlock. Comprehensive decontamination after completion of this work would result in lower dose rates when installing the 3.5-ton crane. When this work was postponed, the 2.4 man-Rem was eliminated from the estimate. This savings largely contributes to the difference between CY estimated and actual doses for May.

\section{Modifications to Procedures}

An example of dose savings attributed to modification of procedures is low-level-waste hauls using the SEG 3-82B cask. The cask is loaded by moving it into the airlock, remotely loading the cask with the waste package, and then removing the cask from the airlock for shipping. The procedure requires two airlock entries, one to insert the cask into the airlock, and one to retrieve the cask.

Changes to the cask loading procedure and increasing worker familiarity with the procedure due to a "campaign"(n) approach resulted in lower personnel dose rates. Additionally, movement of source term materials in the hot cells and increased decontamination activities in the airlock led to generally lower airlock . dose rates. The result of these actions was lower cumulative doses for individual waste-haul evolutions. Historic documentation of doses associated with waste hauls averaged $430 \mathrm{mRem}$ per evolution. The waste campaign accomplished January through April 1995 (22 waste hauls) averaged $250 \mathrm{mRem}$ per load out, or $58 \%$ of the estimated dose (Table 2). This accounts for 2.3 man-Rem savings and largely contributes to the difference between (CY) estimated and actual doses for February and March.

(a) Concentrating a number of waste hauls into a contiguous series rather than doing a waste haul when a load is ready for burial. 
Table 2. Dose from Low-Level Waste Hauls

\begin{tabular}{|c|c|c|c|c|c|c|c|c|c|}
\hline $401 \%$ & $100 \%$ & ol. & 4 & $\cos$ & $=203$ & $1, \ldots$ & $1+2$ & $\ldots$ & - \\
\hline $40047 A$ & 324-95-003 & SEG Cask & $01 / 05 / 95$ & January & 430 & 11.5 & 447 & 12.0 & 1.04 \\
\hline $40047 \mathrm{~B}$ & $324-95-008$ & SEG Load out & $01 / 18 / 95$ & January & 430 & 11.5 & 362 & 9.7 & 0.84 \\
\hline $40047 \mathrm{C}$ & $324-95-013$ & SEG Cask Load Out & $01 / 22 / 95$ & January & 430 & 11.5 & 318 & 8.5 & 0.74 \\
\hline $40047 D$ & $32495-015$ & SEG Cask Load out & $01 / 26 / 95$ & January & 430 & 11.5 & 236 & 6.3 & 0.55 \\
\hline 40047E & $324-95-018$ & SEG Cask Load out & $01 / 28 / 95$ & January & 430 & 11.5 & 205 & 5.5 & 0.48 \\
\hline $40047 \mathrm{H}$ & $32495-020$ & SEG Cask Load out & $02 / 01 / 95$ & February & 430 & 6.0 & 155 & 2.2 & 0.36 \\
\hline 400471 & $324-95-026$ & SEG Cask Load out GC-57 & $02 / 08 / 95$ & February & 430 & 6.0 & 365 & 5.1 & 0.85 \\
\hline $40047 \mathrm{~J}$ & 324-95-027 & SEG Cask Load out GC-61 & $02 / 10 / 95$ & February & 430 & 6.0 & 215 & 3.0 & 0.50 \\
\hline $40047 \mathrm{~K}$ & $324-95-031$ & SEG Load \& Remove SEG Cask with GC-62 & $02 / 19 / 95$ & February & 430 & 6.0 & 250 & 3.5 & 0.58 \\
\hline $40047 \mathrm{~L}$ & $324-95-034$ & SEG Load out GC-68 & $02 / 23 / 95$ & February & 430 & 6.0 & 289 & 4.0 & 0.67 \\
\hline $40047 \mathrm{~N}$ & $324-95-036$ & SEG Load out GC-67 & $02 / 23 / 95$ & February & 430 & 6.0 & 203 & 2.8 & 0.47 \\
\hline 400470 & $324-95-038$ & SEG Load out GC-78 & $03 / 05 / 95$ & February & 430 & 6.0 & 170 & 2.4 & 0.40 \\
\hline $40047 P$ & $324-95-043$ & SEG Load out GC-78 & $03 / 07 / 95$ & March & 430 & 6.2 & 172 & 2.5 & 0.40 \\
\hline $40047 Q$ & $324-95-045$ & SEG Load out GC-79 & $03 / 14 / 95$ & March & 430 & 6.2 & 131 & 1.9 & 0.30 \\
\hline 40048R & $324-95-050$ & SEG Load out GC-77 & $03 / 19 / 95$ & March & 430 & 6.2 & 365 & 5.3 & 0.85 \\
\hline $40047 \mathrm{~S}$ & $324-95-051$ & SEG Load out GC-72 & $03 / 27 / 95$ & March & 430 & 6.2 & 390 & 5.6 & 0.91 \\
\hline $40047 \mathrm{U}$ & $324-95-060$ & SEG/ GC-83 & $04 / 04 / 95$ & April & 430 & 3.4 & 196 & 1.5 & 0.46 \\
\hline $700110 A$ & $324-95-063$ & SEG Load out GC-82 & $04 / 06 / 95$ & April & 194 & 1.5 & 187 & 1.5 & 0.96 \\
\hline $700110 \mathrm{~B}$ & $324-95-065$ & SEG Load - CG81 & $04 / 09 / 95$ & April & 194 & 1.5 & 167 & 1.3 & 0.86 \\
\hline $700110 \mathrm{C}$ & $324-95-067$ & SEG Load out GC-86 & $04 / 17 / 95$ & April & 194 & 1.5 & 247 & 1.9 & 1.27 \\
\hline 7001100 & 324-95-069 & SEG Load out GC-87 & $04 / 23 / 95$ & April & 194 & 1.5 & 216 & 1.7 & 1.11 \\
\hline $700110 E$ & $324-95-074$ & SEG Load out GC-74 & $04 / 26 / 95$ & April & 194 & 1.5 & 207 & 1.6 & 1.07 \\
\hline $700110 \mathrm{G}$ & $324-95-080$ & SEG Load out GC-327-1 & $08 / 05 / 95$ & May & 430 & 5.7 & 463 & 6.1 & 1.08 \\
\hline $700110 \mathrm{~J}$ & $324-95-145$ & SEG Load Out & $08 / 14 / 95$ & August & 430 & 6.8 & 221 & 3.5 & 0.51 \\
\hline & & & & & & & & & \\
\hline & & & & & & & & & \\
\hline & & Estimated Dose for Activities Completed & & & 9140 & & & & \\
\hline & & Act/Actual Est & & & & & & & 0.68 \\
\hline & & Aclual Dose Recelved for Actlvities Completed & & & & & 6177 & & \\
\hline & & $\begin{array}{l}\text { Numbers in italles were not included in the original } \\
\text { CY estimate. They are included here for the sake } \\
\text { of comparison to the actual dose. }\end{array}$ & & & & & & & \\
\hline
\end{tabular}




\section{Reduction of Dose Through Decontamination or Other Means}

\section{Manipulator Changes and Repair Activities}

Implementing ALARA measures, such as rearranging source-term material inside B-Cell and reducing PPE requirements (with resulting increases in worker efficiency), throughout the year resulted in lower personnel dose for manipulator changes. Rearranging source-term material within the cell lowered dose rates at penetrations and access ports around the cell. The planned movement of source term within the cell, most notably repositioning the spent-fuel assemblies ${ }^{(k)}$ from directly in front of the cell doors (located at the east end of the cell) to the west wall, was also a contributing factor in lowering general area dose rates within the airlock. Additionally, modification of requirements for wearing respirators during manipulator changes significantly decreased the amount of time required to remove and install a manipulator. The time savings directly equates to lower worker dose (time, distance, and shielding are the major ALARA considerations). Table 3 is a record of manipulator changes that occurred in CY 1995, showing estimated and actual dose per evolution. The ALARA measures taken resulted in actual dose of $0.6 \mathrm{man}$ Rem below the CY 1995 estimate.

Reductions in actual dose seen for manipulator repair work, although not due strictly to ALARA measures taken, resulted in a savings of 3.3 man-Rem as compared to the estimated dose (see Table 4). Although the estimated dose for this task was judged to be over-conservative, some dose reduction was due to more rigorous decontamination of manipulators before repairing and modifying respiratory protection requirements for some phases of the decontamination and repair work.

(a) This was discussed in PNL-10739, 324 Building Life Cycle Dose Estimates for Planned Work, September 1995. 
Table 3. Dose from Manipulator Changes

\begin{tabular}{|c|c|c|c|c|c|c|c|c|}
\hline Actatos & 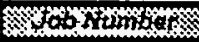 & 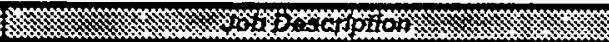 & Norta & Extzoos & 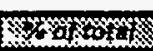 & Action & Wh & 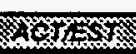 \\
\hline 400999 & 324-95-001 & Manipulator Pull & $01 / 04 / 95$ & 34 & 0.9 & 145 & 3.9 & 4.26 \\
\hline 400565 & $324-95-005$ & Manipulator Pull North \& South MSM B-Cell & $01 / 09 / 95$ & 50 & 1.3 & 22 & 0.6 & 0.44 \\
\hline 400566 & 324-95-006 & Manipulator Pull LH North MSM Installation & $01 / 10 / 95$ & 50 & 1.3 & 10 & 0.3 & 0.20 \\
\hline $400565 B$ & $324-95-010$ & Manipulator Pull, C-Cell & $01 / 17 / 94$ & 34 & 0.9 & 0 & 0.0 & 0.00 \\
\hline 400567 & $324-95-0-40,041$ & Manipulator Pull B-Cell South & $03 / 01 / 95$ & 50 & 0.7 & 5 & 0.1 & 0.10 \\
\hline $400565 C$ & 324-95-044 & Manipulator Pull RH & $03 / 07 / 95$ & 34 & $\overline{0.5}$ & 40 & 0.6 & 1.18 \\
\hline 400565D & 324-95-058 & Manipulator Pull SMF, Station \#3 & $03 / 29 / 95$ & 34 & 0.5 & 5 & 0.1 & 0.15 \\
\hline $40056 \mathrm{E}$ & $324-95-064$ & Manipulator Pull South Window & $04 / 04 / 95$ & 34 & 0.3 & 10 & 0.1 & 0.29 \\
\hline 400569 & 324-95-072 & Manipulator Pull B-Gallery South & $04 / 24 / 95$ & 50 & 0.4 & 10 & 0.1 & 0.20 \\
\hline 400566 & 324-95-084 & Manipulator Pull, SMF Gallery & $05 / 10 / 95$ & 34 & 0.4 & 0 & 0.0 & 0.00 \\
\hline 400570 & 324-95-086 & Manipulator Pull B-Cell & $05 / 11 / 95$ & 50 & 0.7 & 30 & 0.4 & 0.60 \\
\hline $40056 \mathrm{H}$ & 324-95-095 & Manipulator Pull RH & $05 / 25 / 95$ & 34 & 0.4 & 0 & 0.0 & 0.00 \\
\hline 400561 & 324-95-102 & Manipulator Pull LHS & $06 / 05 / 95$ & 34 & 0.2 & 15 & 0.1 & 0.44 \\
\hline \multirow[t]{4}{*}{$40056 \mathrm{~J}$} & 324-95-124 & Manipulator Pull SMF & $07 / 13 / 95$ & 34 & 0.6 & 0 & 0.0 & 0.00 \\
\hline & $324-95-131$ & Manipulator Pull & $07 / 20 / 95$ & 34 & 0.6 & 0 & 0.0 & 0.00 \\
\hline & 324-95-137 & Manipulator Pull SMF & $08 / 01 / 95$ & 50 & 0.8 & 0 & 0.0 & 0.00 \\
\hline & 324-95-141 & Manipulator Pull Alr Lock & $08 / 08 / 95$ & 50 & 0.8 & 12 & 0.2 & 0.24 \\
\hline 400571 & 324-95-152 & Manipulator Pull B-Cell & $08 / 18 / 95$ & 50 & 0.8 & 0 & 0.0 & 0.00 \\
\hline \multirow[t]{2}{*}{400753} & 324-95-161 & Manipulator Pull B-Cell (RH) & $08 / 28 / 95$ & 50 & 0.8 & 8 & 0.1 & 0.16 \\
\hline & 324-95-175 & Manipulator Pull Air Lock & $09 / 08 / 95$ & 30 & 0.6 & 0 & 0.0 & 0.00 \\
\hline \multirow[t]{2}{*}{400572} & 324-95-176 & Manipulator Pull B-Cell LHS \& LHWW & $09 / 09 / 95$ & 50 & 1.0 & 30 & 0.6 & 0.60 \\
\hline & 324-95-177 & Manipulator Pull South Coll & $09 / 11 / 95$ & 30 & 0.6 & 1 & 0.0 & 0.03 \\
\hline 400575 & 324-95-188 & Manipulator Pull South B-Cell & $10 / 04 / 95$ & 30 & 1.7 & 0 & 0.0 & 0.00 \\
\hline 400574 & 324-95-190 & Manipulator Pull LHS B-Cell & $10 / 04 / 95$ & 30 & 1.7 & 2 & 0.1 & 0.07 \\
\hline 400574 & 324-95-191 & Manipulator Pull LHS B-Coll & $10 / 05 / 95$ & 40 & 2 & 4 & 0.2 & 0.10 \\
\hline 400575 & 324-95-220 & Manipulator Pull Dual B-Cell Replacement & $11 / 08 / 95$ & 40 & 1.9 & 17 & 0.8 & 0.43 \\
\hline 400576 & $324-95-221$ & $\begin{array}{l}\text { Manipulator Pull L.H.N. B-Gallery \& resituate R.H.N. } \\
\text { MSM }\end{array}$ & $11 / 09 / 95$ & 40 & 1.9 & 5 & 0.2 & 0.13 \\
\hline 400577 & 324-95-222 & Manipulator Pull RHN MSM & $11 / 10 / 95$ & 40 & 1.9 & 15 & 0.7 & 0.38 \\
\hline 400578 & 324-95-227 & Manipulator Pull RHW MSM & $11 / 14 / 95$ & 40 & 1.9 & 0 & 0.0 & 0.00 \\
\hline 400578 & 324-95-244 & Manipulator Pull RHS - B-Cell gallery & $12 / 12 / 95$ & 40 & 3.3 & 7 & 0.6 & 0.18 \\
\hline BC400579 & $324-95-248$ & Manipulator Pull (2) & $12 / 15 / 95$ & 40 & 3.3 & 7 & 0.6 & 0.18 \\
\hline \multirow[t]{9}{*}{400580} & 324-95-251 & Manipulator Pull B-Gallery, South Window & $12 / 22 / 95$ & 40 & 3.3 & 6 & 0.5 & 0.15 \\
\hline & & a & & & & & & \\
\hline & & $\cdot$ & & & & & & \\
\hline & & & & & & & & \\
\hline & & Estimatod Dose for Activities Completed & & 1280 & & & & \\
\hline & & Act/Actual Est & & & & & & 0.32 \\
\hline & & Actual Dose Rocolved for Activitios Completed & & & & 406 & & \\
\hline & & & & & & & & \\
\hline & . & $\begin{array}{l}\text { Numbers in ltalics were not included in the original } \\
\text { cY estimate. They are included here for the sake of } \\
\text { comparison to the actual dose. }\end{array}$ & & & & & & \\
\hline
\end{tabular}


Table 4. Dose from Manipulator Repair

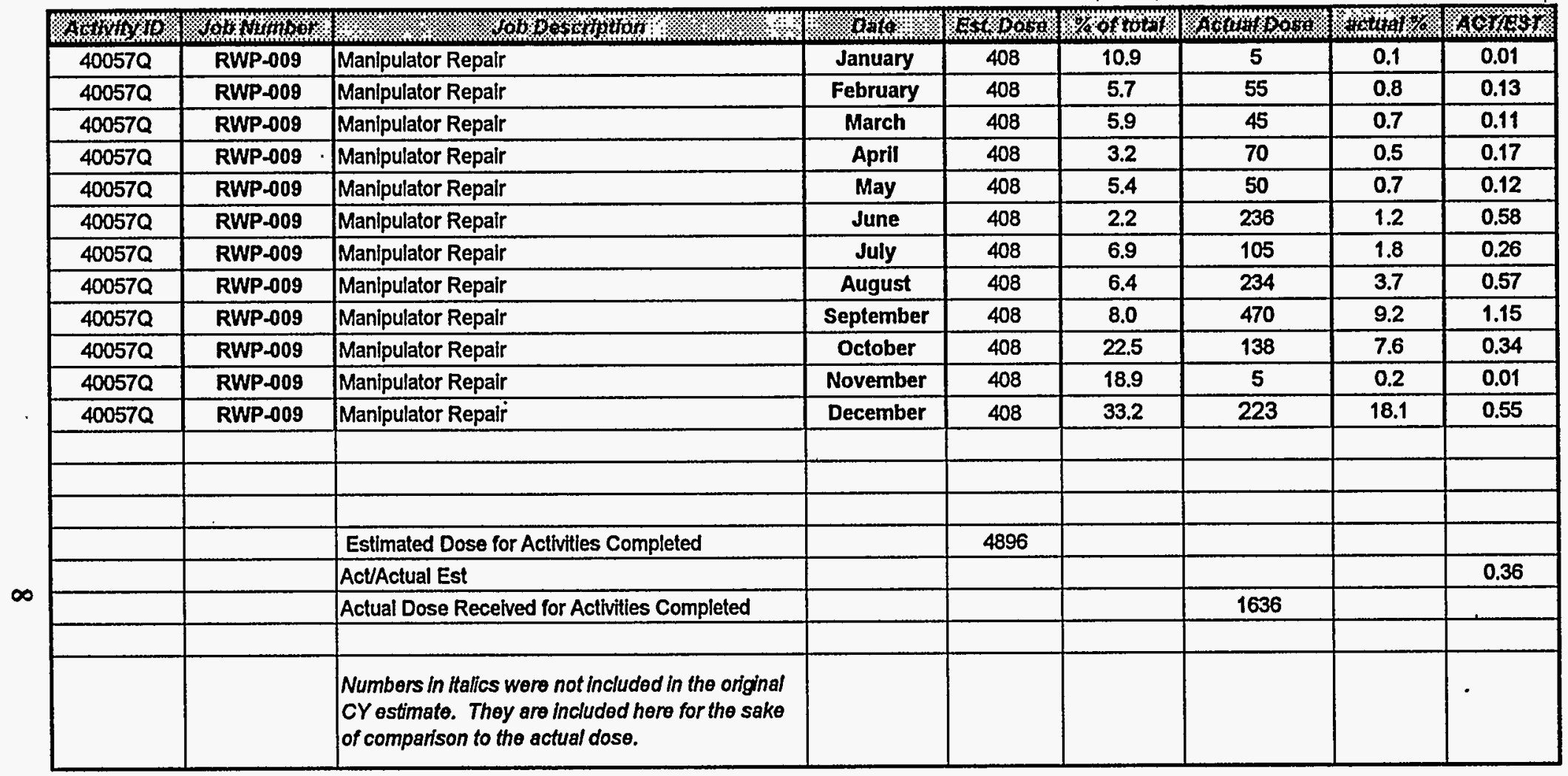




\section{C-Cell Decontamination}

The C-Cell decontamination and crane installation evolutions were well below estimates. Initial estimates for this work were based on historical dose levels seen during the last cell cleanup evolution in 1986. The captive in-cell crane had never been replaced, and some uncertainty existed about the methods required to remove the crane from the rails. This led to conservative time estimates from KEH management for removing the old crane; this factor increased the dose estimates for the work.

To ensure that workers were prepared for any contingency encountered during the crane replacement, extensive mock-up work was done by KEH personnel involved in the job. The mock-up training proved to be very helpful for the actual crane replacement work and reduced the actual entry time in the cell to 6 hours rather than the 24 hours in the estimate.

The use of a new decontamination tool, the Ultra High Pressure (UHP) decontamination system, resulted in dose rates in the cell being less than half of estimated levels. The UHP system delivers a jet of water at a pressure of 50,000 PSI to rotating nozzles. Although the effective decontamination width of the spray is only 2 inches, the spray can be moved at a rapid $(\sim 2 \mathrm{ft} / \mathrm{sec})$ rate, allowing large areas to be decontaminated quickly. The spray apparatus, which is easily handled, allowed the old crane as well as the floor and walls of the cell to be decontaminated to a height of approximately 5 feet.

Overall reductions in actual dose to personnel on this job versus the best estimate was on the order of 12.6 Man-Rem, a reduction of $86 \%$ (Table 5). This accounts for much of the difference between (CY) estimated and actual dose for the month of June.

\section{Total Documented Dose Savings for CY 1995}

As discussed above, dose savings from ALARA actions in the facility were realized through elimination/postponement of tasks (5.5 Man-Rem), procedural changes (2.3 Man-Rem), and reductions in dose rates due to decontamination or other means (16.5 Man-Rem). Total dose savings due to ALARA actions was 24.3 Man-Rem for CY 1995. 
Table 5. Dose from C-Cell Activities

\begin{tabular}{|c|c|c|c|c|c|c|c|c|}
\hline 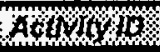 & $\% o b$ oro $6 \%$ & 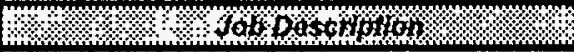 & $\operatorname{pax}$ & Fos & ofof & sotionosive & 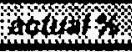 & Ackizsis \\
\hline 502 & $324-95-151$ & C-Cell Survey-Decon-Remove Debris & $08 / 17 / 95$ & 975 & 15.4 & 638 & 10.1 & 0.65 \\
\hline 510 & $\begin{array}{l}324-95-153 \\
125,157,162\end{array}$ & C-Cell Crane Replacement & 08/19/95 & 3300 & 52.0 & 3253 & 51.3 & 0.99 \\
\hline 500 & 324-95-155 & C-Cell REC Air Lock Waste Haul & $08 / 22 / 95$ & 975 & 15.4 & 967 & 15.2 & 0.99 \\
\hline$\overline{502}$ & $324-95-164$ & C-Cell Window Repair & 09/02/95 & 975 & 19.0 & 528 & 10.3 & 0.54 \\
\hline & & & & & & & & \\
\hline & & & & & & & & \\
\hline & & CY Estimated Dose for C-Cell Activities & & 17240 & & & & \\
\hline & & Estimated Dose for Activities Completed & & 6225 & & & & \\
\hline & & Act/Actual Est & & & & & & 0.87 \\
\hline & & Actual Dose Received for Actvitiles Completed & & & & 5386 & & \\
\hline & & Actual/CY Est & & & & & & 0.31 \\
\hline
\end{tabular}




\section{Exposure Goal vs. Exposure Estimates}

The Hot Cell Operations Group exposure goal for CY 1995 was to reduce personnel exposure by 20\% of the CY 1995 estimated dose (73.7 Man-Rem), or 14.7 Man-Rem. As explained above, exposure reduction due to ALARA measures was 24.3 Man-Rem for CY 1995. This represents a reduction in personnel exposure from CY 1995 estimates of 33\%.

A portion of the dose savings, however, was due to postponement or cancellation of scheduled work (3.1 Man-Rem for installation of cell cleaning systems canceled and 2.4 Man-Rem for postponing installation of the 3.5-ton crane). By subtracting the 3.5 Man-Rem reflected by these actions from both the CY estimate (73.7 Man-Rem) and the ALARA measures savings (24.3 Man-Rem), then recalculating the percentage of savings, we arrive at a reduction in personnel exposure from CY 1995 estimates of $28 \%$. This is a more accurate portrayal of ALARA savings, since dose associated with the 3.5-ton crane installation will be taken at a later date, and it could be argued that cancellation of dose is not a true ALARA measure.

\section{Basis of Estimates for CY 1996 Facility Dose Estimate}

Actual dose records for CY 1995 are now historical documents for generating future dose estimates. The basis of estimates for different tasks was generated from this information and is presented in Table 6. These data were used to compile new dose estimates for work scheduled for CY 1996 (see Appendix B estimate for CY 1996). As a result of using these revised bases of estimates, CY 1996 dose estimates should not be as over-conservative as the CY 1995 estimate.

The CY 1996 exposure estimate, based on work schedules in place in December 1995, projects facility exposure estimates for radiation workers at $45.4 \mathrm{Man}-\mathrm{Rem}$. As discussed above, the estimate is based on more recent dose-rate information and reflects procedural changes and dose-rate reductions in the major work area (the REC Airlock). From an Operations standpoint, however, it is felt that a challenging ALARA goal for the calender year would be to reduce a worker dose by $20 \%$. The exposure goal for CY 1996 , based on a $20 \%$ reduction from the estimated dose of $45.4 \mathrm{Man}-\mathrm{Rem}$, is therefore 36.3 Man-Rem. 
Table 6a. CY 1996 Baseline Job Estimates

\begin{tabular}{|c|c|c|c|c|c|c|c|}
\hline \multicolumn{8}{|c|}{ BASIS OF ESTIMATES FOR RADIOLOGICAL JOBS } \\
\hline & & & & \multirow{2}{*}{\multicolumn{3}{|c|}{ JOB B AIRLOCK ENTRY }} & \\
\hline \multicolumn{3}{|c|}{$\begin{array}{l}\text { JOB A AIRLOCK ENTRY } \\
\end{array}$} & & & & & \\
\hline \multirow{2}{*}{\multicolumn{2}{|c|}{$\begin{array}{l}\text { MILLWRIGHT } \\
\text { UNDRESSER }\end{array}$}} & $250 \mathrm{mR}$ & & \multicolumn{2}{|c|}{ ENTRY PERSON } & $100 \mathrm{mR}$ & \\
\hline & & $50 \mathrm{mR}$ & & \multirow{2}{*}{\multicolumn{2}{|c|}{$\frac{\text { UNDRESSER }}{\text { RPT }}$}} & $30 \mathrm{mR}$ & \\
\hline \multicolumn{2}{|c|}{ RPT } & $100 \mathrm{mR}$ & & & & $75 \mathrm{mR}$ & \\
\hline \multicolumn{2}{|c|}{ ASSIST } & $20 \mathrm{mR}$ & & \multicolumn{2}{|c|}{ ASSIST } & $20 \mathrm{mR}$ & \\
\hline & & & & & & & \\
\hline \multicolumn{2}{|c|}{ TOTAL } & $420 \mathrm{mR}$ & & \multicolumn{2}{|c|}{ TOTAL } & $230 \mathrm{mR}$ & \\
\hline \multirow{2}{*}{\multicolumn{3}{|c|}{ JOB C AIRLOCK ENTRY }} & & \multirow{2}{*}{\multicolumn{4}{|c|}{ JOB D 6-TON CRANE REPAIR/PM }} \\
\hline & & & & & & & \\
\hline \multirow{2}{*}{\multicolumn{2}{|c|}{$\begin{array}{l}\text { ENTRY PERSON } \\
\text { UNDRESSER } \\
\end{array}$}} & $150 \mathrm{mR}$ & & \multirow{2}{*}{\multicolumn{2}{|c|}{\begin{tabular}{|l|} 
MILLWRRIGHT \\
ELECTRICIAN
\end{tabular}}} & $1200 \mathrm{mR}$ & \\
\hline & & $30 \mathrm{mR}$ & & & & $600 \mathrm{mR}$ & \\
\hline RPT & & $75 \mathrm{mR}$ & & \multicolumn{2}{|c|}{ UNDRESSER } & $250 \mathrm{mR}$ & \\
\hline \multirow[t]{2}{*}{ ASSIST } & & $25 \mathrm{mR}$ & & \begin{tabular}{|l|} 
RPT \\
\end{tabular} & & $300 \mathrm{mR}$ & \\
\hline & & & & ASSIST & & $30 \mathrm{mR}$ & \\
\hline \multirow[t]{3}{*}{ TOTAL } & & $280 \mathrm{mR}$ & & & & & \\
\hline & & & & TOTAL & & $2380 \mathrm{mR}$ & \\
\hline & & & & & & & \\
\hline \multirow{2}{*}{\multicolumn{2}{|c|}{$\begin{array}{c}\text { JOB E AIF } \\
\text { LABORERS }\end{array}$}} & OCK ENTRY & & \multicolumn{3}{|c|}{ JOB F AIRLOCK ENTRY } & \\
\hline & & $250 \mathrm{mR}$ & & ELECTRIC & IAN & $300 \mathrm{mR}$ & \\
\hline \multicolumn{2}{|c|}{ WELDERS } & $130 \mathrm{mR}$ & & LABORER & & $300 \mathrm{mR}$ & \\
\hline PIPEFITI & RS & $130 \mathrm{mR}$ & & UNDRESS & & $50 \mathrm{mR}$ & \\
\hline UNDRES & ER & $50 \mathrm{mR}$ & & ASSIST & & $25 \mathrm{mR}$ & \\
\hline ASSIST & & $25 \mathrm{mR}$ & & & & & \\
\hline & & & & TOTAL & & $675 \mathrm{mR}$ & \\
\hline TOTAL & & $585 \mathrm{mR}$ & & & & & \\
\hline- & 0205 & & & & & & \\
\hline & $O B G_{5}$ & DN CRANE REP & & JOB & H VAULT & TANK SAN & PLES \\
\hline IRONWO & KEER & $600 \mathrm{mR}$ & & TECHNICI & & $100 \mathrm{mR}$ & \\
\hline LABORE & & $300 \mathrm{mR}$ & & RCT & & $30 \mathrm{mR}$ & \\
\hline UNDRES & ER & $100 \mathrm{mR}$ & & & & & \\
\hline ELECTRI & IAN & $300 \mathrm{mR}$ & & TOTAL & & $130 \mathrm{mR}$ & \\
\hline ASSIST & & $30 \mathrm{mR}$ & & & & & \\
\hline & & & & & & & \\
\hline TOTAL & & $1330 \mathrm{mR}$ & & & & & \\
\hline & B J SAM & F T & & $\overrightarrow{100 K}$ & AIRLOCK & ENTRY & \\
\hline TECHNIC & & $10 \mathrm{mR}$ & & MILLWRIG & HHT & $400 \mathrm{mR}$ & \\
\hline & & & & RCT & & $225 \mathrm{mR}$ & \\
\hline TOTAL & & $10 \mathrm{mR}$ & & UNDRESS & & $75 \mathrm{mR}$ & \\
\hline & & & & \begin{tabular}{|l|} 
ASSIST \\
\end{tabular} & & $30 \mathrm{mR}$ & \\
\hline & & & & & & & \\
\hline & & & & TOTAL & & $730 \mathrm{mR}$ & \\
\hline & & & & & & & \\
\hline & & & & & & & \\
\hline & & & & & & & \\
\hline & & & & & & & \\
\hline & & & & & & & \\
\hline & & & & & & & \\
\hline
\end{tabular}


Table 6b. CY 1996 Baseline Job Estimates

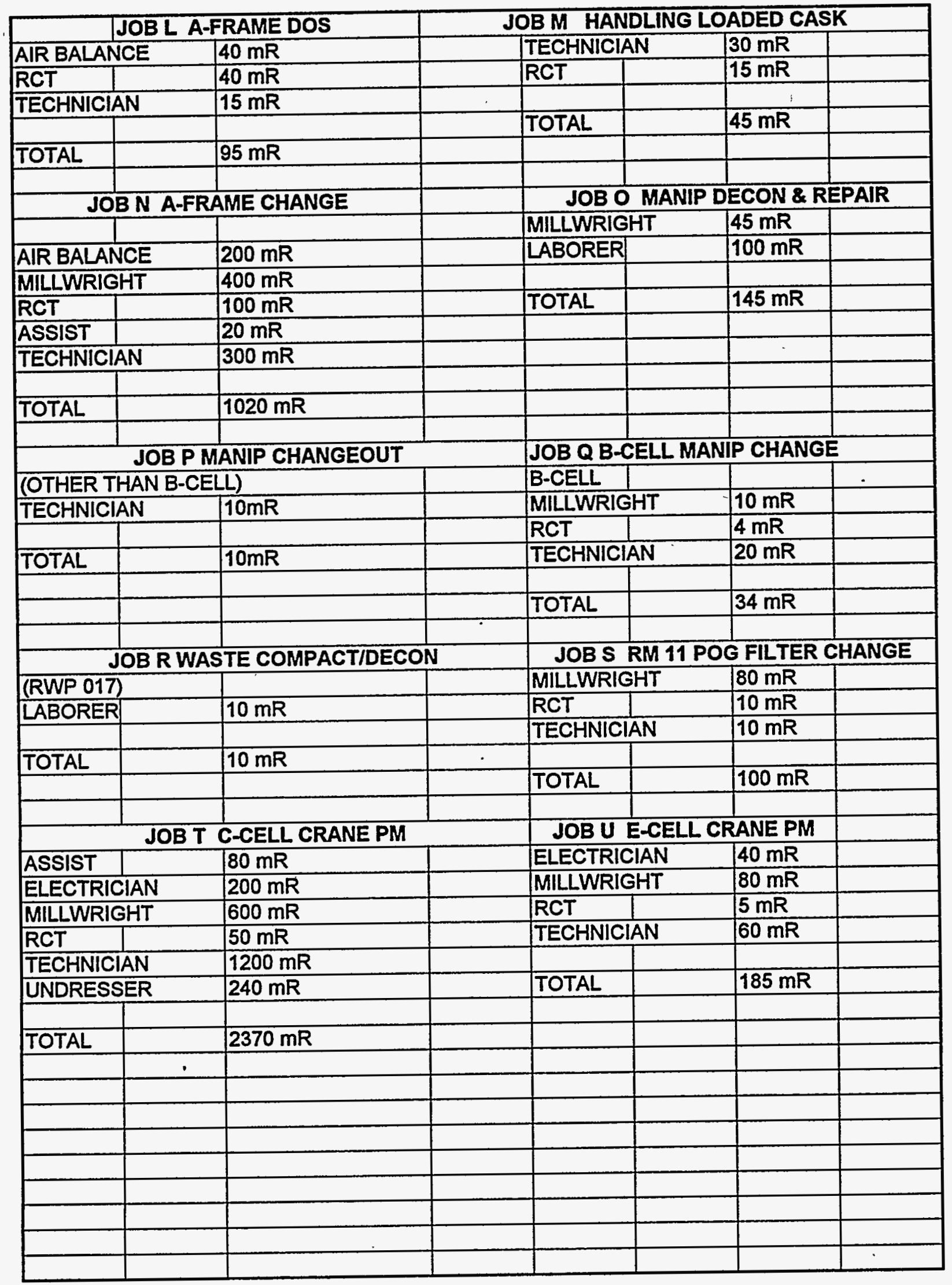


Table 6c. CY 1996 Baseline Job Estimates

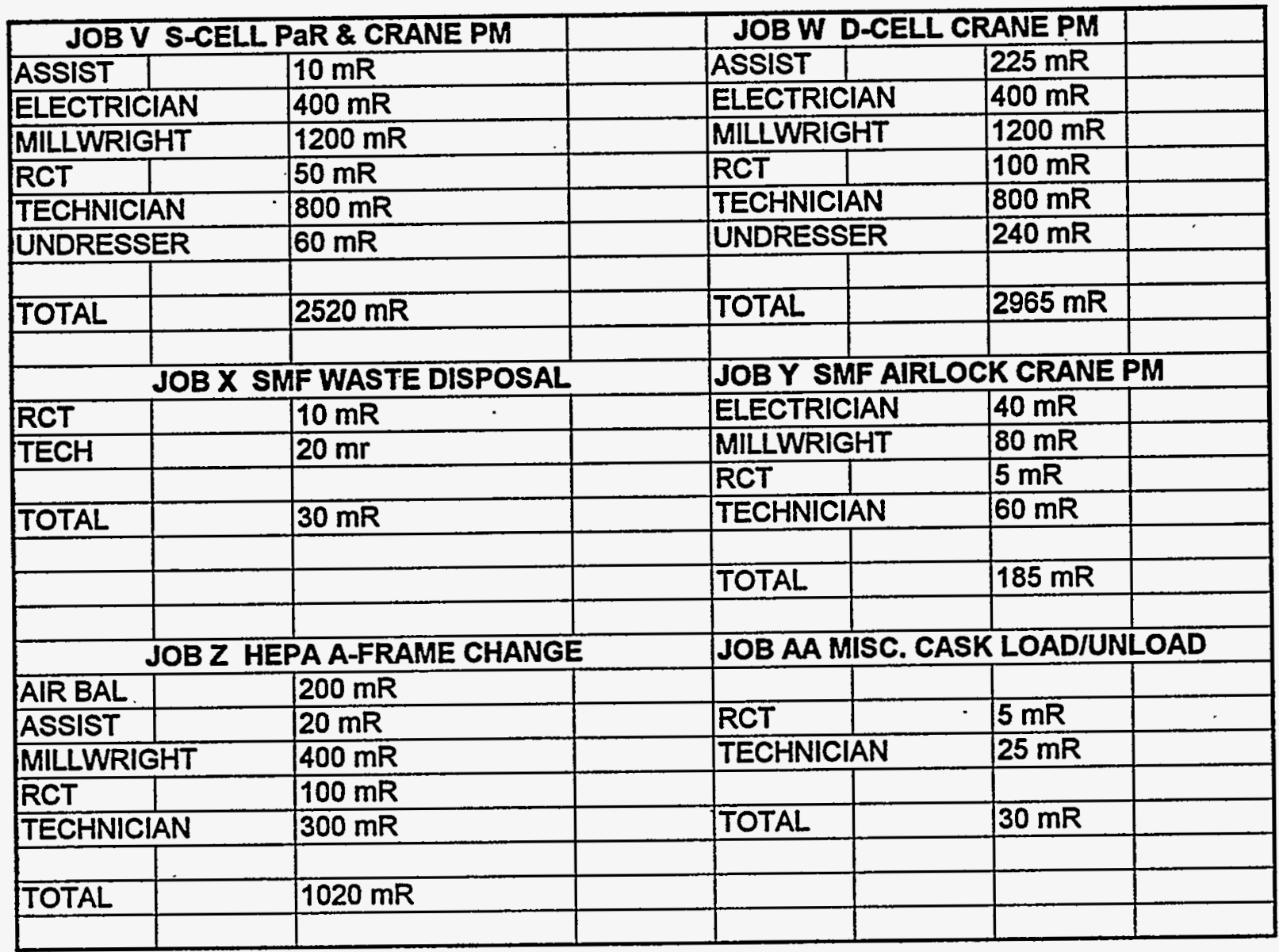




\section{Appendix A}

\section{Comparison by Month of Estimates vs.}

\section{Actuals with Jobs Identified}





\section{Comparison by Month of Estimates vs. Actuals with Jobs Identified}

Each of the following tables contains data for 1 month. If the activity had been included in the dose estimate for the year, the activity identification (ID) number from the data base is included. A blank activity ID number indicates that the activity was not included in the dose estimate for the year. The actual work done each month did not necessarily parallel the schedule from which the estimate was derived. Many tasks were postponed to a later date, and changes in work scope created additional tasks not included in the estimate.

The following pages provide actual dose for job activities that resulted in worker dose. The data were collated from ALARA/pre-job packages prepared for work tasks in the facility. Jobs that were part of the CY 1995 dose estimate were included, as well as jobs for which no estimate was prepared. The actual dose is the secondary dosimetry readings recorded in the packages at the completion of the job. These readings, taken on a cumulative basis, are within $10 \%$ of the record dose for any individual.

In the detailed breakdown, the actual \% column represents a weighted method to compare the significance of the work activity. It represents the proportion of the doses for the activity and the monthly dose from the CY estimate (see Table 1). This gives a relative ranking of the significance of the dose received for that activity.

To weight an activity, the dose associated with the activity is divided by the monthly estimate (see the following tables, one for each month). This \% of total shows the significance of the job in relation to the estimated dose for the month.

The ACT/EST column indicates the relative accuracy of the dose estimate for that specific activity by comparing the actual dose received for the job against the estimate for the job. The estimate is increasingly accurate as the ratio moves closer to one. 
January 95

\begin{tabular}{|c|c|c|c|c|c|c|c|c|}
\hline obos & 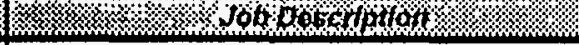 & owito & Monthts & Estoos & orortofo & Aocuaposol & $24 \mathrm{rim}$ & Karms \\
\hline RWP-009 & Manipulator Repalr & January & January & 408 & 10.9 & 5 & 0.1 & 0.01 \\
\hline RWP-017 & Wasto CompactoriDecon & January & January & 10 & 0.3 & $\mathbf{0}$ & 0.0 & 0.00 \\
\hline 324-95-001 & Manipulator Pull & $01 / 04 / 95$ & January & 34 & 0.9 & 145 & 3.9 & 4.28 \\
\hline 324-95-003 & SEG Cask & $01 / 05 / 95$ & January & 430 & 11.5 & 447 & 12.0 & 1.04 \\
\hline $324-95-005$ & Manipulator Pull North \& South MSM B-Cell & $01 / 09 / 95$ & January & 50 & 1.3 & 22 & 0.6 & 0.44 \\
\hline $324-95-006$ & Manlpulator Pull LH North MSM Installation & $01 / 10 / 95$ & January & 50 & 1.3 & 10 & 0.3 & 0.20 \\
\hline $324-95-008$ & SEG Load out & $01 / 18 / 95$ & January & 430 & 11.5 & 362 & 9.7 & 0.84 \\
\hline $324-95-010$ & Manipulator Pull, C-Cell & $01 / 17 / 94$ & January & 34. & 0.9 & 0 & 0.0 & 0.00 \\
\hline $324-95-013$ & SEG Cask Load Out & $01 / 22 / 95$ & January & 430 & 11.5 & 318 & 8.5 & 0.74 \\
\hline $324-95-015$ & SEG Cask Load out & $01 / 26 / 95$ & January & 430 & $\$ 11.5$ & 236 & 6.3 & 0.55 \\
\hline $324-95-018$ & SEG Cask Load out & $01 / 28 / 95$ & January & 430 & 11.5 & 205 & 5.5 & 0.48 \\
\hline & & & & & & & & \\
\hline & CY Estimate-Month of January & & & 3731 & & & & \\
\hline & Estimated Dose for Activitles Completed & & & 2736 & & & & \\
\hline & Act/Actual Est & & & & & & & 0.64 \\
\hline & Actual Dose Recelved for Activities Completed & & & & & 1750 & & \\
\hline & & & & & & & & \\
\hline
\end{tabular}


February 95

\begin{tabular}{|c|c|c|c|c|c|c|c|c|c|}
\hline 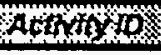 & 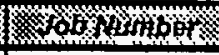 & 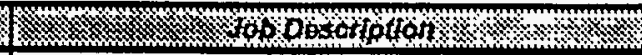 & pot & Hothe & \& 1000 & 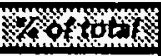 & Actomooto & 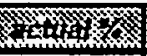 & Kates \\
\hline & RWP-009 & Manipulalor Repalr & February & February & 408 & 5.7 & 55 & 0.8 & 0.13 \\
\hline & RWP-017 & Waste Compactor/Decon & February & February & 20 & 0.3 & 0 & 0.0 & 0.00 \\
\hline \multirow[t]{4}{*}{$40047 \mathrm{H}$} & $324-95-020$ & SEG Cask Load out & $02 / 01 / 95$ & February & 430 & 6.0 & 155 & 2.2 & 0.36 \\
\hline & $324-95-022$ & NLI Cask Loading & $02 / 04 / 95$ & February & 430 & 6.0 & 391 & 5.5 & 0.91 \\
\hline & $324-95-023$ & NLI Cask Load out & $02 / 05 / 95$ & February & 430 & 6.0 & 279 & 3.9 & 0.65 \\
\hline & $324-95-025$ & NLI Cask Load out & $02 / 06 / 95$ & February & 430 & 6.0 & 235 & 3.3. & 0.55 \\
\hline 400471 & 324-95-026 & SEG Cask Load out GC-57 & $02 / 08 / 95$ & February & 430 & 6.0 & 365 & 5.1 & 0.85 \\
\hline $40047 \mathrm{~J}$ & $324-95-027$ & SEG Cask Load out GC-61 & $02 / 10 / 95$ & February & 430 & 6.0 & 215 & 3.0 & 0.50 \\
\hline $40047 \mathrm{M}$ & $324-95-029,30$ & Air Lock Clean Up & $02 / 12 / 95$ & February & 535 & 7.5 & 695 & 9.7 & 1.30 \\
\hline $40047 \mathrm{~K}$ & $324-95-031$ & SEG Load \& Removo SEG Cask with GC-62 & 02/19/95 & February & 430 & 6.0 & 250 & 3.5 & 0.58 \\
\hline 132A95A & $324-95-032$ & Air Lock, REC Camera Repalr & $02 / 15 / 95$ & February & 64 & 0.9 & 60 & 0.8 & 0.94 \\
\hline $40047 \mathrm{~L}$ & 324-95-034 & SEG Load out GC-68 & $02 / 23 / 95$ & February & 430 & 6.0 & 289 & 4.0 & 0.67 \\
\hline \multirow[t]{2}{*}{$40047 \mathrm{~N}$} & 324-95-036 & SEG Load out GC-67 & $02 / 23 / 95$ & February & 430 & 6.0 & 203 & 2.8 & 0.47 \\
\hline & 324-95-037 & Air Lock Place GC-80 in the Air Lock & $02 / 24 / 95$ & February & 0 & 0.0 & 0 & 0.0 & \\
\hline 400470 & $324-95-038^{-}$ & SEG Load out GC-78 & $03 / 05 / 95$ & February & 430 & 6.0 & 170 & 2.4 & 0.40 \\
\hline \multirow[t]{8}{*}{400025} & 324-95-039 & Air Lock/Cell Door PMs & $02 / 28 / 95$ & February & 1815 & 25.4 & 532 & 7.5 & 0.29 \\
\hline & & & & & & & & & \\
\hline & & CY Estimate-Month of February & & & 7137 & & & $\cdot$ & \\
\hline & & Estlmated Dose for Acthitles Completed & & & 7142 & & & & \\
\hline & & Act/Actual Est & & & & & & & 0.55 \\
\hline & & Actual Dose Recelved for Activitles Completed & & & & & 3894 & & \\
\hline & & & & & & & & & \\
\hline & & $\begin{array}{l}\text { Numbers in Halles woro not included in the orlgnal } \\
\text { cY estmate. They are included here for the sake } \\
\text { of compantson to the actual dose. }\end{array}$ & & & & - & & & \\
\hline
\end{tabular}


MARCH 95

ESTIMATES vS ACTUALS

\begin{tabular}{|c|c|c|c|c|c|c|c|c|c|}
\hline Activity 10 & Job Number & Job Descriptlon & Date & Month & Est Dose & $\%$ of total & Actual Dose & actual \% & ACT/EST \\
\hline & RWP-009 & Manipulator Repair & March & March & 408 & 5.8 & 45 & 0.7 & 0.11 \\
\hline & RWP-017 & Waste Compactor/Decon & March & March & 20 & 0.3 & 5 & 0.1 & 0.25 \\
\hline 400567 & $324-95-040,041$ & Manipulator Pull B-Cell South & $03 / 01 / 95$ & March & 50 & 0.7 & 5 & 0.1 & 0.10 \\
\hline $40047 P$ & $324-95-043$ & SEG Load out GC-78 & $03 / 07 / 95$ & 'March & 430 & 6.2 & 172 & 2.5 & 0.40 \\
\hline $400565 C$ & 324-95-044 & Manlpulator Pull RH & $03 / 07 / 95$ & March & 34 & 0.5 & 40 & 0.6 & 1.18 \\
\hline $40047 Q$ & $324-95-045$ & SEG Load out GC-79 & $03 / 14 / 95$ & March & 430 & 6.2 & 131 & 1.9 & 0.30 \\
\hline $40048 R$ & $324-95-050$ & SEG Load out GC-77 & $03 / 19 / 95$ & March & 430 & 6.2 & 365 & 5.3 & 0.85 \\
\hline 400475 & $324-95-051$ & SEG Load out GC-72 & $03 / 27 / 95$ & March & 430 & 6.2 & 390 & 5.6 & 0.91 \\
\hline 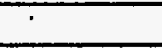 & $324-95-056$ & Open Air Lock Door & $03 / 23 / 95$ & March & 0 & 0.0 & 0 & 0.0 & \\
\hline 28190 & $324-95-057$ & Room 11 Fitter Chango & $03 / 24 / 95$ & March & 100 & 1.4 & 89 & 1.3 & 0.89 \\
\hline \multirow[t]{10}{*}{4005650} & 324-95-058 & Manipulator Pull SMF, Station \#3. & $03 / 29 / 95$ & March & 34 & 0.5 & 5 & 0.1 & 0.15 \\
\hline & $324-95-059$ & NLI & $03 / 29 / 95$ & March & 430 & 6.2 & 537 & 7.8 & 1.25 \\
\hline & & $\cdot$ & & & & & & & \\
\hline & & & & & & & & & \\
\hline & & CY Estimate-Month of March & & & 6922 & & & & \\
\hline & & Estimated Dose for Acthities Completed & & & 2796 & & & & \\
\hline & & Act/Actual Est & & & & & & & 0.64 \\
\hline & & Actual Dose Recelved for Activitles Completed & & & & & 1784 & $\cdot$ & \\
\hline & & (2. & & & & & & & \\
\hline & & $\begin{array}{l}\text { Numbers In Halles were not included in the } \\
\text { original CY ostimato. They are included here for } \\
\text { the sake of comparison to the actual dose. }\end{array}$ & & & & & & & \\
\hline
\end{tabular}


April 95

\begin{tabular}{|c|c|c|c|c|c|c|c|c|c|}
\hline ACA J Y IO & 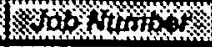 & otodscrodrdn & onte & 4hotitis & Estoost & * of tot & sction of & II & Hets \\
\hline & RWP-009 & Manipulator Repair & April & April & 408 & 3.2 & 70 & 0.5 & 0.17 \\
\hline & RWP-017 & Waste Compactor/Decon & April & April & 20 & 0.2 & 0 & 0.0 & 0.00 \\
\hline $40047 U$ & $324-95-060$ & SEG/ GC-83 & $04104 / 95$ & April & 430 & 3.4 & 196 & 1.5 & 0.46 \\
\hline $40047 x$ & $324-95-061$ & Air Lock Clean up & $04 / 02 / 95$ & April & 321 & 2.5 & 410 & 3.2 & 1.28 \\
\hline $700110 \mathrm{~A}$ & $324-95-063$ & SEG Load out GC-82 & $04 / 06 / 95$ & April & 194 & 1.5 & 187 & 1.5 & 0.96 \\
\hline $40056 \mathrm{E}$ & $324-95-064$ & Manlpulator Pull South Window & $04 / 04 / 95$ & April & 34 & 0.3 & 10 & 0.1 & 0.29 \\
\hline $700110 \mathrm{~B}$ & $324-95-065$ & SEG Load - CG81 & $04 / 09 / 95$ & April & 194 & 1.5 & 167 & 1.3 & 0.86 \\
\hline $700110 \mathrm{C}$ & $324-95-067$ & SEG Load out GC-86 & $04 / 17 / 95$ & April & 194 & 1.5 & 247 & 1.9 & 1.27 \\
\hline $700110 \mathrm{D}$ & $324-95-069$ & SEG Load out GC-87 & $04 / 23 / 95$ & April & 194 & 1.5 & 216 & 1.7 & 1.11 \\
\hline $40056 \mathrm{~F}$ & $324-95-070$ & Manipulator Pull C-Gallery & $04 / 19 / 95$ & April & 34 & 0.3 & 20 & 0.2 & 0.59 \\
\hline 400569 & $324-95-072$ & Manipulator Pull B-Gallery South & $04 / 24 / 95$ & April & 50 & 0.4 & 10 & 0.1 & 0.20 \\
\hline 500315 & $324-95-073$ & A-Frame DOS & $04 / 24 / 95$ & April & 30 & 0.2 & 45 & 0.4 & 1,50 \\
\hline \multirow[t]{5}{*}{$700110 \mathrm{E}$} & $324-95-074$ & SEG Load out GC-74 & $04 / 26 / 95$ & April & 194 & 1.5 & 207 & 1.6 & 1.07 \\
\hline & & CY Estimate-Month of April & & & 12801 & & & & \\
\hline & & Estimated Dose for Activities Completed & & & 2322 & & & & \\
\hline & & Act/Actual Est & & & & & & & 0.77 \\
\hline & & Actual Dose Recelved for Activities Completed & & & & & 1785 & & \\
\hline
\end{tabular}

$-n$
-
-
$\cdots$ 


\section{May 95}

\begin{tabular}{|c|c|c|c|c|c|c|c|c|c|}
\hline 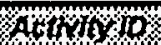 & $\%$ \% & 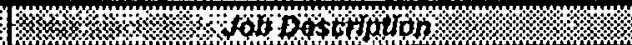 & 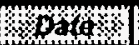 & Moonte & Hot posts & \%otorit & 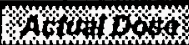 & 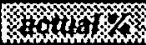 & 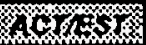 \\
\hline & RWP.009 & Manipulator Repair & May & May & 408 & 5.4 & 50 & 0.7 & 0.12 \\
\hline & RWP-017 & Waste Compactor/Decon & May & May & 20 & 0.3 & 0 & 0.0 & 0.00 \\
\hline 28191 & $324-95-078$ & Room 11 - PM & $05 / 03 / 95$ & May & 110 & 1.5 & 5 & 0.1 & 0.05 \\
\hline \multirow[t]{3}{*}{$700110 G$} & $324-95-080$ & SEG Load out GC-327-1 & 05/05/95 & May & 430 & 5.7 & 463 & 6.1 & 1.08 \\
\hline & $324-95-081$ & Air Lock Clean Up - NLI & 05/06/95 & May & 430 & 5.7 & 775 & 10.2 & 1.80 \\
\hline & $324-95-082$ & NLI Cask Pull & $05 / 08 / 95$ & May & 430 & 5.7 & 225 & 3.0 & 0.52 \\
\hline 40056G & $324-95-084$ & Manipulator Pull, SMF Gallery & $05 / 10 / 95$ & May & 34 & 0.4 & 0 & 0.0 & 0.00 \\
\hline 400570 & 324-95-086 & Manipulator Pull B-Cell & $05 / 11 / 95$ & May & 50 & 0.7 & 30 & 0.4 & 0.60 \\
\hline \multirow[t]{6}{*}{$40056 \mathrm{H}$} & $324-95-095$ & Manipulator Pull RH & $05 / 25 / 95$ & May & 34 & 0.4 & 0 & 0.0 & 0.00 \\
\hline & & & & & & & & & \\
\hline & & CY Estimate-Month of May & & & 7584 & & & & \\
\hline & & Estimated Dose for Activitles Completed & & & 1946 & & & & \\
\hline & & Act/Actual Est & & & & & & & 0.80 \\
\hline & & Actual Dose Recelved for Activitles Completed & & & & & 1548 & & \\
\hline & & & & & & & & & \\
\hline D) & & $\begin{array}{l}\text { Numbers in Halcs wore not inctuded in the original } \\
\text { cY estimato. They are included horo for the sake } \\
\text { of comparison to the actual dose. }\end{array}$ & & & & & & & \\
\hline
\end{tabular}




\begin{tabular}{|c|c|c|c|c|c|c|c|c|c|}
\hline & & & & & & & & &. \\
\hline & & 6929 & & & & & 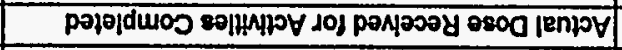 & & \\
\hline \multirow[t]{5}{*}{ SLOO } & & & & & & & 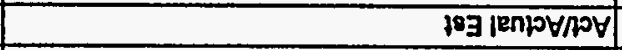 & & \\
\hline & & & & $26 \varepsilon 8$ & & & 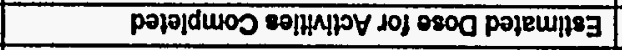 & & \\
\hline & & & & 60681 & & & 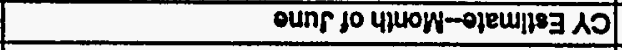 & & \\
\hline & & & & & & & & & \\
\hline & & & & & & & & & \\
\hline $69 \%$ & 乙`9l & 6SOE & 96 & St8L & oun & S6/62/90 & 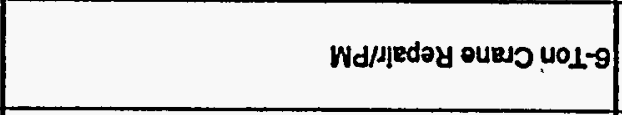 & 9นเ-S6-tZE & $\begin{array}{l}\text { HSGZO' } \\
\text { '9SSZOt } \\
\text { ' } 9800 \text { S }\end{array}$ \\
\hline$\$ 80$ & $L " t$ & $\varepsilon 88$ & $9 \mathbf{s}$ & OSOL & ounp & S6r92/90 & 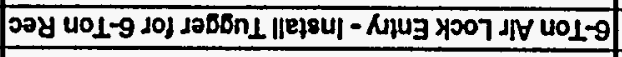 & SLL'十LL-S6-tZE & 0980009 \\
\hline zE'0 & $\angle ’ 9$ & Z9ZІ & 0.12 & SL6E & ounp & S6EZ/9O & 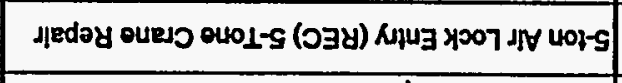 & 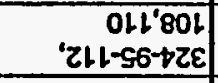 & 8SLPOOP \\
\hline$\varepsilon+1$ & 0.6 & $\$ 9 L$ & 82 & SES & จunr & S6/ZL/90 & 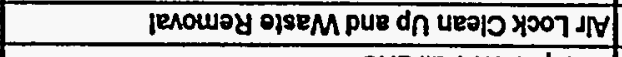 & tol-s6-tZE & YSSZOP \\
\hline$\$ 0$ & 1.0 & st & 20 & te & euns & S6/SO/90 & SHT Ind dojejndịuew & $201-56 \rightarrow 2 \varepsilon$ & $19500 t$ \\
\hline $60^{\circ} 0$ & $\varepsilon$ & OS & $0 \cdot \varepsilon$ & 095 & oun & S6/20/90 & dois jsna $\mathrm{N}$ IIEjsul - Rlu & $660-s 6 \rightarrow 2 \varepsilon$ & so\& \\
\hline$\infty 0^{\prime} 0$ & 0.0 & $\mathbf{0}$ & 10 & 02 & ouns & oun & Losog/10poduros opseM & LLO-dMy & \\
\hline 890 & $2 !$ & $\theta \varepsilon z$ & 22 & $80 t$ & ouns & oune & Jeday Jojeindjuew| & $600 \mathrm{dM} \mathrm{y}$ & \\
\hline LSZ/LOY & 96 Jemos & esog jumov & $10901 / 0 \%$ & $\operatorname{esog} 259$ & 4uOH & ojeg & uopdposeg gor & soqunN qor & al хумвов \\
\hline
\end{tabular}

S6 בNก 
ESTIMATES VS ACTUALS

\begin{tabular}{|c|c|c|c|c|c|c|c|c|c|}
\hline Activity ID & Job Number & Job Description & Date & Month & Est Dose & \% of total & Actual Dose & ectual \%. & ACT/EST \\
\hline & RWP-009 & Manlpulator Repalr & July & July & 408 & 6.9 & 105 & 1.8 & 0.26 \\
\hline & RWP-017 & Waste Compactor/Decon & July & July & 20 & 0.3 & 35 & 0.8 & 1.75 \\
\hline $40255 \mathrm{C}$ & 324-95-119 & Alr Lock Clean UpWaste Load out \& H/L Decon. & $07 / 08 / 95$ & July & 535 & 9.1 & 255 & 4.3 & 0.48 \\
\hline 40255D & $324-95-121$ & Air Lock Clean Up Entry (REC) Decon & $07 / 11 / 95$ & July & 535 & 9.1 & 304 & 5.2 & 0.57 \\
\hline $50086 \mathrm{~B}$ & $324-95-122$ & 6-Ton Crane decon/repalr & $07 / 13 / 95$ & July & 750 & 12.8 & 499 & 8.5 & 0.67 \\
\hline $40056 \mathrm{~J}$ & $324-95-124$ & Manlpulator SMF MSM Pulle & $07 / 13 / 95$ & July & 34 & 0.6 & 0 & 0.0 & 0.00 \\
\hline $40255 E$ & $324-95-126$ & 6-Ton Crane LLW Load out & $07 / 14 / 95$ & July & 535 & 9.1 & 720 & 12.2 & 1.35 \\
\hline \multirow[t]{2}{*}{$40255 \mathrm{~K}$} & $324-95-127$ & Air Lock Entry Repair Blue Heron Crano & $07 / 17 / 95$ & July & 535 & 9.1 & 355 & 6.0 & 0.68 \\
\hline & $324-95-131$ & Manipulator Pull & $07 / 20 / 95$ & July & 34 & 0.6 & 0 & 0.0 & 0.00 \\
\hline \multirow[t]{2}{*}{$40255 \mathrm{~F}$} & 324-95-132 & Alr Lock Clean Up Entry & $07 / 24 / 95$ & July & 535 & 9.1 & 1245 & 21.2 & 2.33 \\
\hline & $324-95-133$ & 6-Ton Crane Trolley Motor Replacement & $07 / 26 / 95$ & July & 1815 & 30.9 & 1535 & 26.1 & 0.85 \\
\hline \multirow[t]{6}{*}{$40255 \mathrm{~J}$} & 324-95-136 & Air Lock Fuel Storage Rack Install & $07 / 31 / 95$ & July & 535 & 9.1 & 341 & 5.8 & 0.64 \\
\hline & & & & & & & & & \\
\hline & & CY Estimato-Month of July & & & 5881 & & & & \\
\hline & & Estimated Dose for Actlvities Completed & & & 6271 & & & & \\
\hline & & Act/Actual Eot & & & & & & & 0.86 \\
\hline & & Actual Dose Recelved for Activities Completed & & & & & 5394 & & \\
\hline & & & & & & & & & \\
\hline
\end{tabular}


AUGUST 95

ESTIMATES VS ACTUALS

\begin{tabular}{|c|c|c|c|c|c|c|c|c|c|}
\hline Activity ID & Job Number & Job Description & Date & Month & Est. Dase & K of total & Actual Dose & actual $\%$ & ACTIEST \\
\hline & RWP-009 & Manipulator Repair & August & August & 408 & 6.4 & 234 & 3.7. & 0.57 \\
\hline & RWP-017 & Waste Compactor/Decon & August & August & 20 & 0.3 & 32 & 0.5 & 1.60 \\
\hline & $324-95-137$ & Manipulator Pull SMF & $08 / 01 / 95$ & August & 50 & 0.8 & 0 & 0.0 & 0.00 \\
\hline & $324-95-138$ & Room 11 P.M.S. & $08103 / 95$ & August & 100 & 1.6 & 0 & 0.0 & 0.00 \\
\hline & $324-95-141$ & Manipulator Pull Alr Lock & $08 / 08 / 95$ & Auguast & 50 & 0.8 & 12 & 0.2 & 0.24 \\
\hline $700110 \mathrm{~J}$ & 324-95-145 & SEG Load Out & $08 / 14 / 95$ & August & 430 & 6.8 & 221 & 3.5 & 0.51 \\
\hline $700110 \mathrm{~L}$ & $324-95-146$ & Alr Lock Clean Up LLW Load Out & $08 / 12 / 95$ & August & 215 & 3.4 & 559 & 8.8 & 2.60 \\
\hline 502 & $324-95-151$ & C-Cell Survey-Decon-Removo Debris & $08 / 17 / 95$ & August & 975 & 15.4 & 638 & 10.1 & 0.65 \\
\hline 400571 & $324-95-152$ & Manipulator Pull B-Cell & $08 / 18 / 95$ & August & 50 & 0.8 & $\mathbf{0}$ & 0.0 & 0.00 \\
\hline 510 & $\begin{array}{l}324-95-153,125, \\
157,162\end{array}$ & C-Cell Crane Replacement & $08 / 19 / 95$ & August & 3300 & $\mathbf{5 2 . 0}$ & 3253 & 51.3 & 0.99 \\
\hline 500 & $324-95-155$ & C-Cell REC Alr Lock Waste Haul & $08 / 22 / 95$ & August & 975 & 15.4 & 967 & 15.2 & 0.99 \\
\hline 400753 & $324-95-161$ & Manipulator Pull B-Cell (RH) & $08 / 28 / 95$ & August & 50 & 0.8 & 8 & 0.1 & 0.16 \\
\hline \multirow[t]{8}{*}{500086} & $324-95-163$ & 6-Ton Crano Survey & $08 / 31 / 95$ & August & 750 & 11.8 & 889 & 14.0 & 1.19 \\
\hline & & & & & & & & & \\
\hline & & & & & & & & & \\
\hline & & CY Estimate-Month of August & & & 6346 & & & & \\
\hline & & Estimated Doso for Acthities Completed & & & 7373 & & & & \\
\hline & & Act/Actual Est & & & & & & & 0.92 \\
\hline & & Actual Dose Recelved for Acthitles Completed & & & & & 6813 & & \\
\hline & & & & & & & & & \\
\hline
\end{tabular}




\section{SEPTEMBER 95}

ESTIMATES vs ACTUALS

\begin{tabular}{|c|c|c|c|c|c|c|c|c|c|}
\hline Activity ID & Job Number & Job Descriptlon & Date & Month & Est, Dose & sof total & Actual Dose & actual \% & ACT/EST \\
\hline & RWP.009 & Manipulator Repair & September & September & 408 & 8.0 & 470 & 9.2 & 1.15 \\
\hline & RWP-017 & Waste Compactor/Decon & September & September & 20 & 0.4 & 11 & 0.2 & 0.55 \\
\hline 502 & $324-95-164$ & C-Cell Window Repair & $09 / 02 / 95$ & September & 975 & 19.0 & 528 & 10.3 & 0.54 \\
\hline 500086 & 324-95-166 & 6-Ton Crane Electrical Repair & $09 / 05 / 95$ & September & 750 & 14.6 & 596 & 11.6 & 0.79 \\
\hline \multirow[t]{2}{*}{315} & $324-95-168$ & Air Lock Entry Change Dust Stop & $09 / 06 / 95$ & September & 560 & 10.9 & 176 & 3.4 & 0.31 \\
\hline & $324-95-171$ & Air Lock Clean Up Survey & $09 / 07 / 95$ & September & 530 & 10.3 & 704 & 13.7 & 1.33 \\
\hline \multirow[t]{2}{*}{901380} & $324-95-174$ & Arr Lock Shielded Door PMs - REC & $09 / 11 / 95$ & September & 1815 & 35.4 & 703 & 13.7 & 0.39 \\
\hline & 324-95-175 & Manipulator Pull Air Lock & $09 / 08 / 95$ & September & 30 & 0.6 & 0 & 0.0 & 0.00 \\
\hline \multirow[t]{8}{*}{400572} & 324-95-176 & Manipulator Pull B-Cell LHS \& LHW & $09 / 09 / 95$ & September & 50 & 1.0 & 30 & 0.6 & 0.60 \\
\hline & $324-95-177$ & Manipulator Pull South Cell & $09 / 11 / 95$ & September & 30 & 0.6 & 1 & 0.0 & 0.03 \\
\hline & & & & & & & & & \\
\hline & & CY Estimato-Month of September & & & 5132 & & & & \\
\hline & & Estimated Dose for Actluttles Completed & & & 5168 & & & & \\
\hline & & Act/Actual Est & & & & & & & 0.62 \\
\hline & & Actual Dose Recetved for Activitles Completed & & & & & 3219 & & \\
\hline & & & & & & & & & \\
\hline
\end{tabular}


OCTOBER 95 .

ESTIMATES vS ACTUALS

\begin{tabular}{|c|c|c|c|c|c|c|c|c|c|}
\hline Activity ID & Job Number & Job Dascription & Date & Month & Est Dose & St of total & Actural Dose & actual $\%$ & ACTIEST \\
\hline & RWP-009 & Manipulator Repalr & October & October & 408 & 22.5 & 138 & 7.6 & 0.34 \\
\hline & RWP-017 & Waste Compactor/Decon : & October & October & 20 & 1.1 & 15 & 0.8 : & 0.75 \\
\hline 400575 & $324-95-188$ & Manipulator Pull South B-Ce!l & $10 / 04 / 95$ & October & 30 & 1.7 & $\mathbf{0}$ & 0.0 & 0.00 \\
\hline 400574 & $324-95-190$ & Manipulator Pull LHS B-Cell & $10 / 04 / 95$ & October & 30 & 1.7 & 2 & 0.1 & 0.07 \\
\hline 400574 & $324-95-191$ & Manipulator Pull LHS B-Cell & $10 / 05 / 95$ & October & 40 & 2 & 4 & 0.2 & 0.10 \\
\hline 402550 & $324-95-192$ & Alr Lock Clean Up Waste Load out & $10 / 05 / 95$ & October & 535 & 29.5 & 393 & 21.7 & 0.73 \\
\hline $600220 A$ & $324-95-193$ & Air Lock PT- Surveys, Pipe Trench Covers & $10 / 06 / 95$ & October & 444 & 24.5 & 113 & 6.2 & 0.25 \\
\hline 402551 & $324-95-195$ & Alr Lock Clean Up Entry - Re-arrange & $10 / 09 / 95$ & October & 535 & 29.5 & 225 & 12.4 & 0.42 \\
\hline 6002208 & $324-95-196$ & Alr Lock PT Clean Up Entry Prep. for Block Plug R & $10 / 11 / 95$ & October & 444 & 24.5 & 267 & 14.7 & 0.60 \\
\hline $600220 C$ & 324-95-199 & Arr Lock PT Cover Blocks & $10 / 12 / 95$ & October & 444 & 24.5 & 829 & 45.7 & 1.87 \\
\hline $700110 \mathrm{~F}$ & $324-95-201$ & Alr Lock Entry to Retrieve Fitter Box & $10 / 17 / 95$ & October & 289 & 15.9 & 252 & 13.9 & 0.87 \\
\hline 500315 & 324-95-202 & A-Frame & $10 / 16 / 95$ & October & 30 & 1.7 & 56 & 3.1 & 1.87 \\
\hline $132 \mathrm{~A} 95 \mathrm{~J}$ & 324-95-204 & Manipulator Decon (Mele) & 10/18/95 & October & 64 & 3.5 & 15 & 0.8 & 0.23 \\
\hline $40255 \mathrm{H}$ & $324-95-205$ & Air Lock Clean Up Entry to Set Up Sump Pump & $10 / 18 / 95$ & October & 535 & 29.5 & 470 & 25.9 & 0.88 \\
\hline \multirow[t]{7}{*}{$\begin{array}{c}600220 \mathrm{D}, \mathrm{E} \\
40255 \mathrm{~J}\end{array}$} & $324-95-211$ & Arr Lock PT Clean Up (LLW Load-Out) & $10 / 23 / 95$ & October & 1060 & 58 & 3433 & 189 & 3.24 \\
\hline & & & & & & & & & \\
\hline & & CY Estimate-Month of October & & & 1815 & & & & \\
\hline & & Estimated Dose for Activitles Completed & & & 4908 & & & & \\
\hline & & Act/Actual Est & & & & & & & $1.27^{-}$ \\
\hline & & Actual Dose Recelved for Activitles Completed & & & & & 6212 & & \\
\hline & & & & & & & & & \\
\hline
\end{tabular}


NOVEMBER 95

ESTIMATES VS ACTUALS

\begin{tabular}{|c|c|c|c|c|c|c|c|c|c|}
\hline Actlvity 10 & Job Number & Job Descriptlon & Date & Month & Est Dose & Sh of total & Actual Dose & actual \% & ACT/EST \\
\hline & RWP-009 & MANIPULATOR REPAIR & November & November & 408 & 18.8 & 5 & 0.2 & 0.01 \\
\hline & RWP-017 & WASTE PACKAGING/COMPACTING & November & November & 20 & 0.9 & 5 & 0.2 & 0.25 \\
\hline $40058 \mathrm{~J}$ & $324-95-218$ & Manlpulator Pull - SMF & $11 / 06 / 95$ & November & 10 & 0.5 & 2 & 0.1 & 0.20 \\
\hline 400575 & $324-95-220$ & Manlpulator Pull Dual B-Cell Replacement & $11 / 08 / 95$ & November & 40 & 1.9 & 17 & 0.8 & 0.43 \\
\hline 400576 & 324-95-221 & Manipulator Pull L.H.N. B-Gallery \& resituate R.H. & $11 / 09 / 95$ & November & 40 & 1.9 & 5 & $0.2^{\circ}$ & 0.13 \\
\hline 400577 & 324-95-222 & Manipulator Pull RHN MSM & $11 / 10 / 95$ & November & 40 & 1.9 & 15 & 0.7 & 0.38 \\
\hline 400578 & 324-95-227 & Manlpulator Pull RHW MSM & $11 / 14 / 95$ & November & 40 & 1.9 & $\mathbf{0}$ & 0.0 & 0.00 \\
\hline \multirow[t]{9}{*}{$40047 X$} & $324-95-228$ & Air Lock PT Clean Up LLW -ALL Prep. for Pipe Tre & $11 / 15 / 95$ & November & 321 & 14.9 & 345 & 16.0 & 1.07 \\
\hline & 324-95-229 & DOS "A" Frame & $11 / 14 / 95$ & November & 100 & 4.6 & 151 & 7.0 & 1.51 \\
\hline & & & & & & & & & \\
\hline & & & & & & & & & \\
\hline & & CY Estimate-Month of November & & & 2161 & & & & \\
\hline & & Estimated Dose for Acthitties Completed & & & 1019 & & & & \\
\hline & & Act/Actual Est & & & & & & & 0.53 \\
\hline & & Actual Dose Received for Activities Completed & & & & & 545 & & \\
\hline & & & & & & $\cdot$ & $\cdot$ & & \\
\hline$\vec{N}$ & & $\begin{array}{l}\text { Numbers in Halles wore not Includod in the } \\
\text { original } \mathrm{Cr} \text { ostimate. Thoy aro includod horo for } \\
\text { the sake of comparison to the actual dose. }\end{array}$ & & & & & & & \\
\hline
\end{tabular}




\section{DECEMBER 95}

ESTIMATES vS ACTUALS

\begin{tabular}{|c|c|c|c|c|c|c|c|c|c|}
\hline Activity ID & Job Number & Job Description & Date & Month & Est Dose & Kof total & Actural Dose & actual $\%$ & ACT/EST \\
\hline $40057 Q$ & RWP-009 & Manipulator Repair & December & December & 408 & 33.2 & 223 & 18.1 & 0.55 \\
\hline 5000016 & RWP-017 & Waste Compactor/Decon & December & December & 20 & 1.6 & 2 & 0.2 & 0.10 \\
\hline $40255 \mathrm{~J}$ & 324-95-235 & Air Lock Clean Up LLW -Repair Turntablo Wiring & $12 / 01 / 95$ & December & 535 & 43.5 & 620 & 50.4 & 1.16 \\
\hline 600220A & 324-95-241 & Air Lock PT to remove covertlocks & $12 / 07 / 95$ & December & 420 & 34.1 & 486 & 39.5 & 1.16 \\
\hline 400578 & 324-95-244 & Manipulator Pull RHS - B-Cell gallery & $12 / 12 / 95$ & December & 40 & 3.3 & 7 & 0.6 & 0.18 \\
\hline 600910 & 324-95-245 & 5-Ton Arr Lock Cleanout for Crane PM & $12 / 12 / 95$ & December & 305 & 24.8 & 463 & 37.6 & 1.52 \\
\hline $400415 B$ & 324-95-246 & 5-Ton AD Crane PM & $12 / 13 / 95$ & December & 1600 & 130.1 & 1067 & 86.5 & 0.67 \\
\hline BC400579 & $324-95-248$ & Manipulator Pull (2) & 12/15/95 & December & 40 & 3.3 & 7 & 0.6 & 0.18 \\
\hline 400580 & 324-95-251 & Manipulator Pull B-Gallery, South Window & $12 / 22 / 95$ & December & 40 & 3.3 & 6 & 0.5 & 0.15 \\
\hline \multirow[t]{8}{*}{ 6030NA } & 324-95-253 & Install Neutron Analyzer & $12 / 28 / 95$ & December & 200 & 16.3 & 194 & 15.8 & 0.97 \\
\hline & & & & & & & & & \\
\hline & & & & & $\dot{-}$ & & & & \\
\hline & & CY Estimate-Month of December & & & 1230 & & & & \\
\hline & & Estimated Dose for Acthitles Completed & & & 3608 & & & & \\
\hline & & Act/Actual Est & & & & & & & 0.85 \\
\hline & & Actual Dose Recelved for Acthitles Completed & & & & & 3075 & & \\
\hline & & $\begin{array}{l}\text { Numbors in Halles were not includod in the } \\
\text { original CY ostimate. Thoy aro Included hero for } \\
\text { tho sake of comparison to tho actual dose. }\end{array}$ & & & & & & & $\cdot$ \\
\hline
\end{tabular}





\section{Appendix B}

324 Facility Radiation Dose Estimates for CY 1996 



\section{Appendix B Contents}

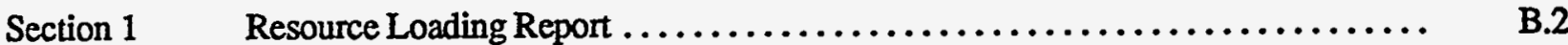

Section $2 \quad$ Resource Loading Summaries ............................ B.10

Section $3 \quad$ Resource Loading Detail by Activity PNNL .................... B.14

Section $4 \quad$ Resource Loading Detail by Resource PNNL $\ldots \ldots \ldots \ldots \ldots \ldots \ldots \ldots \ldots \ldots$ B.36

Section $5 \quad$ Resource Loading Detail by Resource KEH $\ldots \ldots \ldots \ldots \ldots \ldots \ldots \ldots \ldots \ldots$ B.48

Section $6 \quad$ Resource Loading Detail by Activity KEH $\ldots \ldots \ldots \ldots \ldots \ldots \ldots \ldots \ldots \ldots \ldots$. . . . . . 


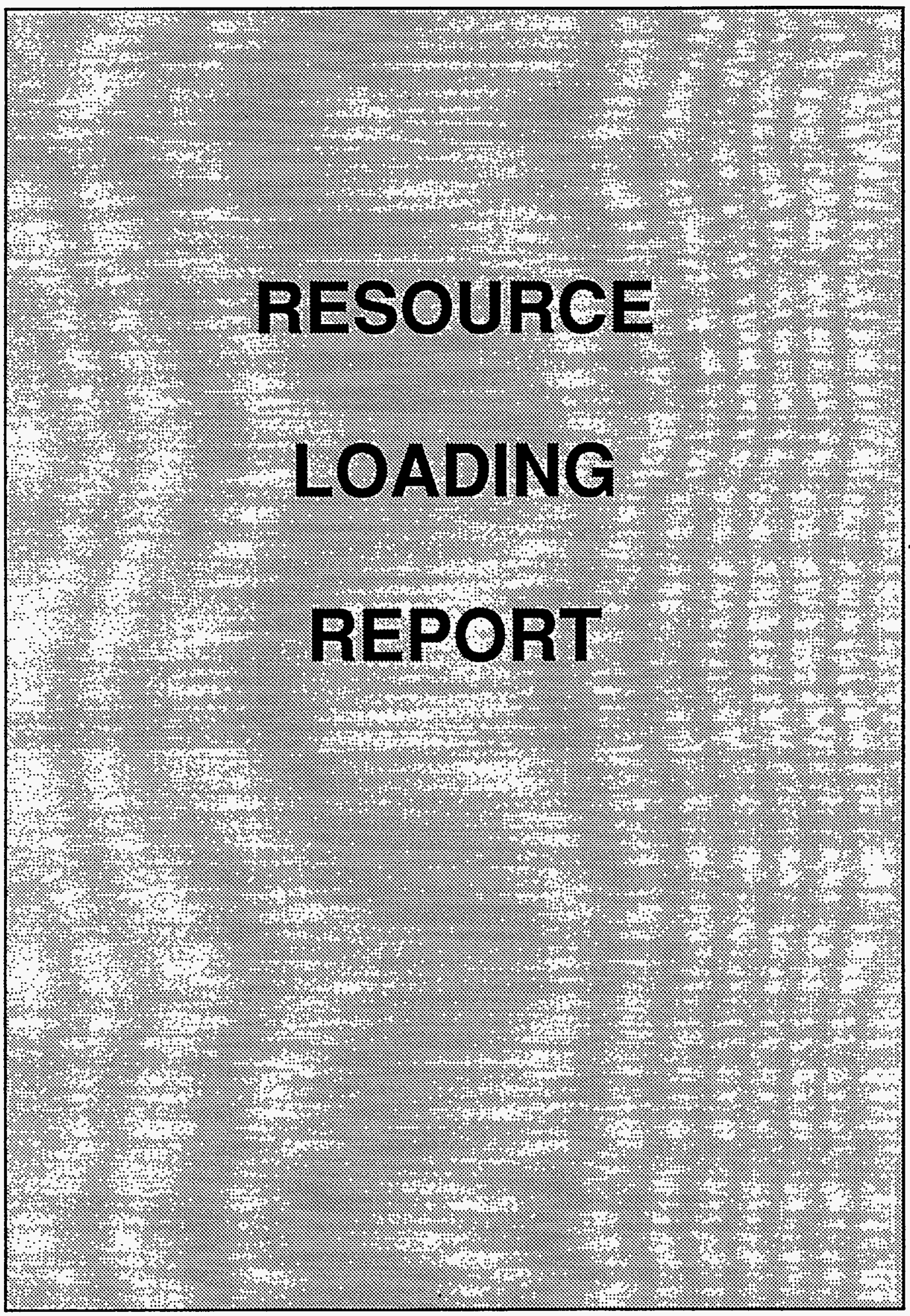

B. 2 
PRINALEAA FROJECT PLANAER

REPORT DATE 12EEC95 FUN NO. 195

RESOURCE LORDING REPORT

START DATE OISAN95 FIN DATE 3 $13: 27$

TOTAL USAGE FOR MONHA

RESOURCE LCADING - TCTAT MONTHEY SUMQATY

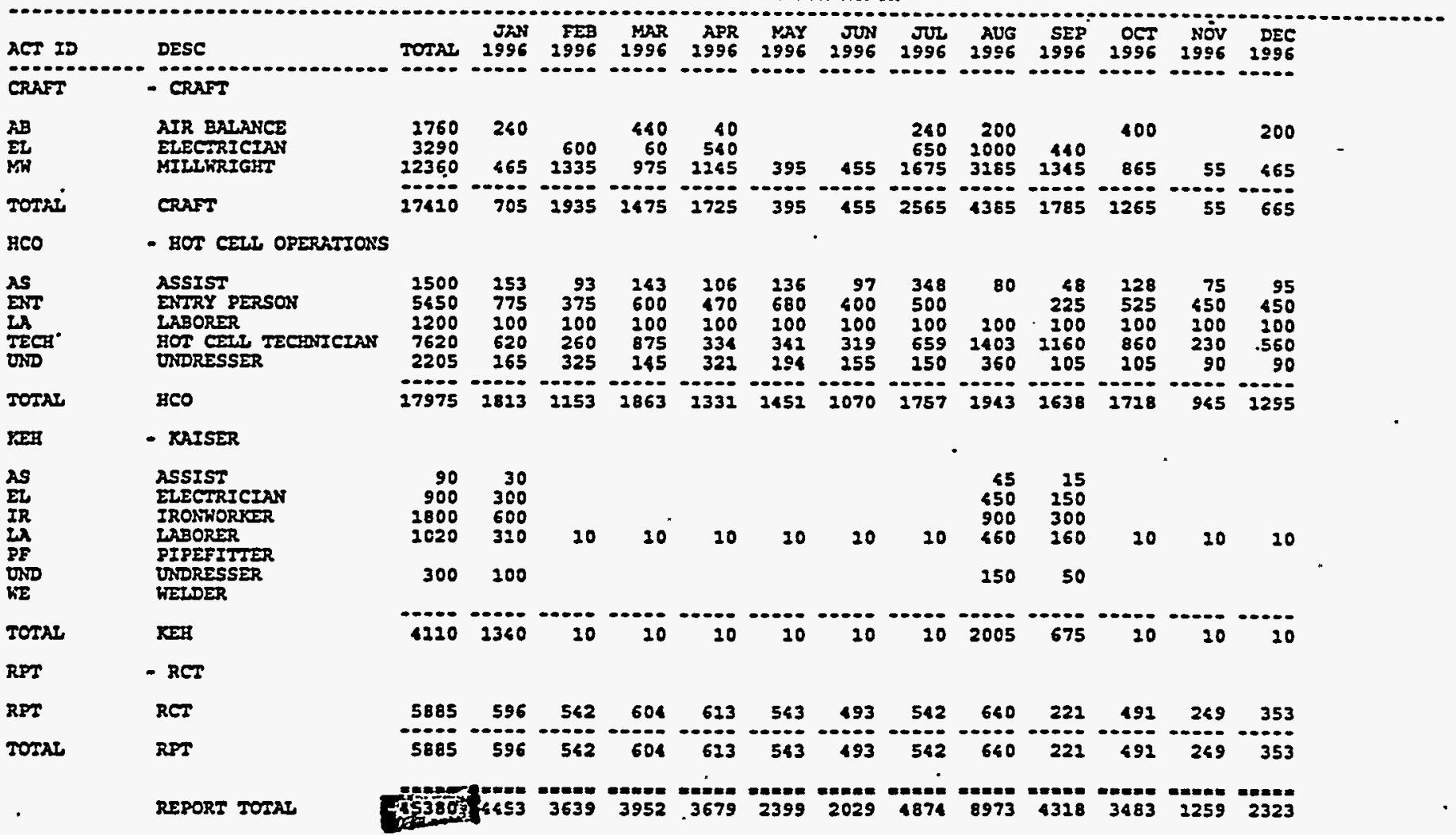

B.3 


\begin{tabular}{|c|c|c|c|c|c|}
\hline \multirow{2}{*}{\multicolumn{2}{|c|}{ 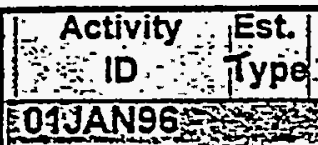 }} & $\therefore \quad$ Activity 1 & $\begin{array}{l}\text { Planned } \\
\text { Start }\end{array}$ & $\begin{array}{l}\text { Total } \\
\text { Dose }\end{array}$ & $\begin{array}{l}\text { Original } \\
\text { duration }\end{array}$ \\
\hline & & 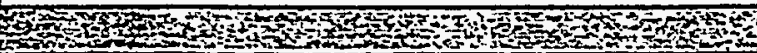 & SThes & 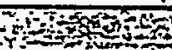 & $203 y^{3}=$ \\
\hline$B C D 313$ & $\mathrm{Cl}_{1}$ & Loadout 3rd G.C. REFR Brick in Aillock & OTJAN96 & 280.00 & 2 \\
\hline BCT4120 & Gi & INSTALI 3-1/2 TONCRANE & O2JAN96 & $1,330.00$ & 7 \\
\hline SFT08 & $x \mid$ & Waste Disposal & 02JAN96* & 30.001 & $T$ \\
\hline $8 C 186$ & $R$ & Waste Compaction/Decon & O3JAN96 & 70.00 & T \\
\hline STV324 & $A A_{1}$ & Misc. Cask Load/UnIoad & O3JAN96 & 30.001 & $T$ \\
\hline BCD405 & $C_{1}^{1}$ & Loadout of Melter GIass in Ainock & OAJAR96 & 280.00 & $T$ \\
\hline BCT5O & Q & Manipulator Changeouts & TOJAN96 & 34.001 & $T$ \\
\hline SM150 & 1 & Manipulator Changeouts & TOJAN96 & 70.00 & $T$ \\
\hline $\sin 326$ & $\overline{A A_{j}}$ & Misc. Cask Load/Unioad & TOJATN95 & $30.00 \div$ & $T$ \\
\hline BCPX4776 & $\bar{c}$ & J st Loadout of High Dose Metals in Airock (scw) & T2JAN96 & 280.00 & 2 \\
\hline$R L \quad 33$ & 8 & Tnstall Vacuum Tank in D-Cell & T5JAIN95 & 225.00 & 2 \\
\hline SM328 & AA & Misc. Cask Load/Unioad & 15JAN96 & 30.00 & 1 \\
\hline BCT3T & 0 & Manipulator Decon and Repair & TEJAN96. & 745.00 & $T$ \\
\hline BCE659 & BB & Moving Waste for NDA & TEJAR96 & 100.00 & $T$ \\
\hline PM260 & Lif & A-Frames & 20JAN96"- & $95.00:$ & $T$ \\
\hline BCPX47TT & $C_{1}$ & Loadt of Miter Feed/liq Mit Seal/Oil Abs in Airlk & 22JAN96 & $280.00 i$ & 2 \\
\hline $\sin 330$ & $\overline{A A_{j}}$ & Misc. Cask Load/Unload & 22JAN96 & 30.00 & $T$ \\
\hline $3 C 2224$ & 21 & HEPA A-Frame DOS & 29JAN96 & $7,020.001$ & $T$ \\
\hline SM332 & $\overline{A A_{i}}$ & Misc. Cask Load/Unioad & 29JAN95 & 30.00 & $T$ \\
\hline BC152 & $Q_{i}$ & Manipulator Changeouts & उOJAN96 & 34.00 & .7 \\
\hline STM152 & $P j$ & Manipulator Changeouts & 30JAN96 & 70.00 & $T$ \\
\hline BCPX4741 & $c_{1}^{2}$ & Tst Loadout of Refractory in Ainlock & 31JAN95 & 280.00 & 2 \\
\hline O1FEB96 & 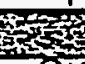 & $b^{3}$ & 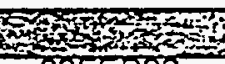 & 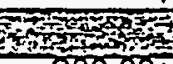 & Pris \\
\hline BCPX4721 & C & Ist Loadout of Dispersible in Airlock & O2FEB96 & 280.00 & 2 \\
\hline $8 C 188$ & $R_{i}$ & Waste Compaction/Decon & OSFEB95 & 70.00 & $T$ \\
\hline STM334 & AA & Misc. Cask LoadJUnIoad & O5FEB95 & 30.00 & $T$ \\
\hline$B C 28193$ & si & RMTIPOG Filier Changeout & OБFEB96 & 370.00 & $T$ \\
\hline BCPX4736 & Cl: & 2nd Loadout of Dispersible in Airock & OEFEB96 & 280.00 & 2 \\
\hline$B C=90040$ & DI & 5th CRAINE MAINTENANCE-FY96 & OTFEB9S & $2,380.00$ & 5 \\
\hline SFT09 & $x$ & Waste Disposal & T2FEB96 & 30.00 & $T$ \\
\hline STM336 & $A A_{j}$ & Misc. Cask LoadJunioad & T2FEB96 & 30.00 & $T$ \\
\hline BC6570 & $\mathrm{BB}$ & Moving Waste for NDA & T6FEBS6 & 700.00 & 1 \\
\hline $\mathrm{BC} 132$ & ण & Manipulator Decon and Repair & 20FEB96 & 145.00 & $T$ \\
\hline$B C 754$ & Q1 & Manipulator Changeouts & 20FEB96 & 34.00 & 1 \\
\hline ST154 & P & Manipulator Changeouts & 2OFEB96 & 10.00 & 7 \\
\hline STM338 & AA & Misc. Cask Load/Unioad & 20FEB96 & 30.00 & $T$ \\
\hline 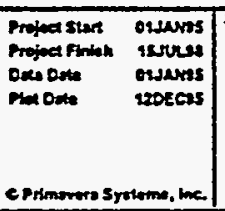 & & $\begin{array}{c}1996324 \text { FACILITY DOSE ESTIMAT } \\
\text { Calendar Year }\end{array}$ & & & \\
\hline
\end{tabular}




\begin{tabular}{|c|c|c|c|c|c|}
\hline $\begin{array}{l}\text { Activity } \\
\text { ID }\end{array}$ & $\begin{array}{l}\text { Est. } \\
\text { Type }\end{array}$ & $\therefore \quad \therefore \quad \therefore$ Description & $\begin{array}{l}\text { Planned } \\
\because \text { Start }\end{array}$ & $\begin{array}{l}\text { Total } \\
\text { Dose }\end{array}$ & $\begin{array}{l}\text { Original } \\
\text { duration }\end{array}$ \\
\hline $\sin 340$ & $A A_{1}$ & Misc. Cask Loaduntoad & 27FEB96 & 30.00 & 1 \\
\hline \multicolumn{6}{|c|}{ 01MAR96E， } \\
\hline BC190 & $R_{\mid}$ & Waste Compaction/Decon & OAMAR96 & 10.00 & 1 \\
\hline SM342 & $A A_{i}$ & Misc. Cask LoadrUnioad & 04MAR96 & 30.00 & $T$ \\
\hline BCSP2000 & $c_{i}^{1}$ & Shipment \#1 Load and Decon Overpacks & O5MAR96 & 280.00 & 6 \\
\hline BC156 & $Q_{1}$ & Manipulator Changeouts & O8MAR96 & 34.00 & $T$ \\
\hline SMT56 & $P$ & Manipulator Changeouts & OBMAR96 & 70.00 & $T$ \\
\hline SIM344 & $\mathrm{AA}_{1}$ & Misc. Cask Load/Unioad & TIMAR96 & 30.00 & $T$ \\
\hline $\mathrm{BC} 133$ & $\sigma_{1}$ & Manipulator Decon and Repalr & T3MAR95 & 745.00 & 7 \\
\hline BCSP2010 & $\mathrm{C}^{-}$ & Shipment \#f Prep, Load, andLeak Test Cask & T3MAR96 & 280.00 & 4 \\
\hline SFTHO & $x_{1}$ & Waste Disposal & T3MAR96 & 30.00 & 7 \\
\hline$B C 60037$ & & WASTE CAMPATGN\# 6 (SEP95) & T5MAR96 & 280.00 & 4 \\
\hline PIV268 & & Annual HEPA filfer PM & T6MAR96 & 95.00 & $T$ \\
\hline BCE671 & $B B$ & Moving Waste for NDA & T8MARP5 & 100.00 & T \\
\hline $\sin 346$ & $A A_{1}$ & TMisc. Cask Load/Unioad & T8MAR96 & 30.00 & $T$ \\
\hline PM312 & $\mathbf{N}_{1}$ & D-Cell A-Frame cable repair & T9MAR95 & $7,020.00$ & 1 \\
\hline SM348 & $\mathrm{AA}_{1}$ & Misc. Cask Load/Unioad & 27MAR96 & 30.00 & 7 \\
\hline SMT58 & $P_{i}$ & iManipulator Changeouts & 28MAR96 & 70.00 & $T$ \\
\hline$B C 50200$ & & CRATE MAINTENANCE & 29MAR96 & $2,380.00$ & 70 \\
\hline BCD323 & $c_{i}$ & SEG Casak Shipment LIner \#?? & 29MARR95 & 280.00 & 1 \\
\hline PM310 & $N_{1}$ & B-Cell A-Frame change & 29MAR $95^{\circ}$ & $7,020.00$ & $T$ \\
\hline \multirow{2}{*}{\multicolumn{6}{|c|}{ 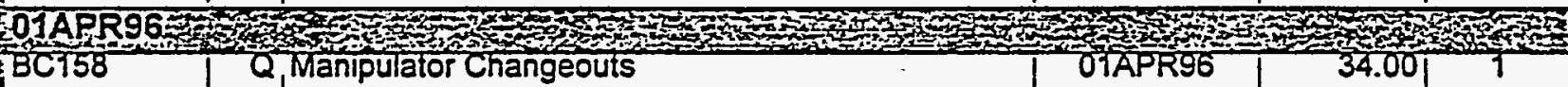 }} \\
\hline & & & & & \\
\hline PN 20 & $m_{1}$ & Receive 29FuelPins from ANL-W@ 324 & OAAPR96" & 45.00 & 3 \\
\hline BC192 & $R_{1}$ & Waste CompactiondDecon. & 03APR96 & 10.00 & 7 \\
\hline 514350 & $\mathrm{AA}_{1}$ & Misc. Cask Load/Unioad & O3APR96 & 30.00 & $T$ \\
\hline PN 21 & $M$ & Transfer 9 ACO-3 Pins from 324 to 327 & 0दAPR96 & 45.00 & 30. \\
\hline SM352 & $A A_{i}$ & Misc. Cask Loaduñoad & TOAPR95 & 30.00 & 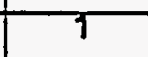 \\
\hline BCSP2020 & $\mathrm{C}$ & Shipment \#2 Load, Decon, and Leak Test & T2APR96 & 280.00 & 6 \\
\hline BC160 & $Q_{1}$ & Manipulator Changeouts & 16APR96 & 34.00 & $T$ \\
\hline SMT60 & $\mathbf{P}$ & Manipulator Changeouts & TEAPR95 & 10.00 & $T$ \\
\hline BCD327 & ci & SEG Cask Shipment /Liner \#? & T7APR95 & 280.00 & 9 \\
\hline SFTT & $x_{1}^{\prime}$ & Waste Disposal & T7APR96" & 30.00 & $T$ \\
\hline STM354 & AA & Misc. Cask Loadrunioad & T7APR9S & 30.00 & T \\
\hline BC6672, & $\mathrm{BB}$ & Moving Waste for NDA & T8APR96 & 100.00 & $T$ \\
\hline PM262 & 4 & A-frames & 20APR96: & 95.00 & $T$ \\
\hline BCF34 & ot & Manipulator Decon and Repair & 22APR96 & .145 .00 & T \\
\hline BCSP2025 & C! & Shipment \#2 Prep, Load, and Leak Test Cask & 22APR96 & 280.00 & 4 \\
\hline SM356 & AA & Misc. Cask Load/Unioad & 29APR96 & 30.00 & $T$ \\
\hline HL $2 \sigma$ & $\mathrm{B}$ & Installn Cellequip. \& gallery services & उOAPR'96 & 225.00 & 5 \\
\hline
\end{tabular}




\begin{tabular}{|c|c|c|c|c|c|}
\hline $\begin{array}{l}\text { Activity } \\
\text { ID }\end{array}$ & $\begin{array}{l}\text { Est. } \\
\text { Type }\end{array}$ & \begin{tabular}{cc}
$\vdots$ & Activity \\
\hdashline & Description
\end{tabular} & $\begin{array}{l}\text { Planned } \\
\text { Start }\end{array}$ & $\begin{array}{l}\text { Total } \\
\text { Dose : }\end{array}$ & $\begin{array}{l}\text { Original } \\
\text { duration }\end{array}$ \\
\hline BCSP2035 & & Shipment \#3 Load, Decon, and Leak Test & OTMAY96 & -280.00 & 6 \\
\hline PN 23 & $M i$ & Store C-T Shipment at 324 & OTMAY95 & 45.00 & 69 \\
\hline BC794 & & Waste Compaction/Decon & T3MAY95 & 10.00 & $T$ \\
\hline PN 114 & $\frac{1}{M i}$ & Xfer Pkgd Fuel Pins to G-Cell or SMF for cing & T3MAY96" & 45.00 & 20 \\
\hline SM358 & AA & Misc. Cask Loadunioad & 03 MAY95 & 30.00 & T \\
\hline BCT62 & $Q$ & Manipulator Changeouts & OEMAY96 & 34.00 & $T$ \\
\hline SMT62 & & Manipulator Changeouts & OEMAY96 & 10.00 & ? \\
\hline $8 C 28194$ & & RMTTPOG Filter Changeout & O7MAY96 & 710.00 & T \\
\hline BCSP2040 & & Shipment \#3 Prep, Load, and Leak Test Cask & T9MAY96 & 280.00 & 4 \\
\hline ST360 & $\frac{1}{A A_{1}}$ & Misc. Cask LoadJUnioad & TOMAY96 & 30.00 & $T$ \\
\hline$B C 5673$ & $\mathrm{BB}_{1}$ & Moving Waste for NDA & T6IVAY96 & 700.001 & $T$ \\
\hline $\mathrm{BCT22}$ & $\sigma_{1}^{1}$ & Manipulator Decon and Repair & T7MAY96 & 145.00 & $T$ \\
\hline BCSP2050 & $c_{i}^{1}$ & Shipment \#4 Load, Decon, and Leak Test & T7MAY96 & 280.00 & 6 \\
\hline SFT12 & $x_{1}^{1}$ & Waste Disposal & TIMAY96" & $30.00 ;$ & 1 \\
\hline $\sin 362$ & $\frac{1}{A A_{1}}$ & Misc. Cask Loadunnioad & TTMAY96 & 30.00 & $T$ \\
\hline$B C 164$ & $Q_{i}$ & Manipulator Changeouts & 23MAY95 & 34.00 & $T$ \\
\hline ST164 & $P_{1}^{1}$ & Manipulator Changeouts & 23MAY96 & 70.00 & T \\
\hline SM364 & $\frac{1}{A A_{1}}$ & Misc. Cask Load/Unioad & 24MAY95 & 30.00 & $\pi$ \\
\hline BCSP2055 & $c_{1}^{1}$ & Shipment \#4 Prep, Load, and Leak Test Cask & 27MAY95 & 280.00 & 4 \\
\hline $3 C 600230$ & $\frac{1}{A_{1}}$ & SPRAY DOWIN COVERBLOCKS \& REINSTALL & 28MAY95 & 420.00 & 2 \\
\hline 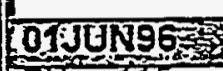 & 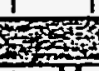 & 1 罗 & 9aph & 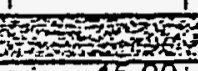 & Penting \\
\hline$P N-\frac{115}{115}$ & M & Transter Packaged fuel to SMF @ 324 & $03 J 01 \times 9 \sigma^{\circ}$ & 45.00 & 2 \\
\hline $\sin 365$ & AA & Misc. Cask Loaduntoad & 03JUN96 & 30.00 & $T$ \\
\hline $8 C 196$ & $R_{1}$ & Waste CompactiondDecon & 0दJUN96 & 10.00 & $T$ \\
\hline BCSP2055 & $c_{j}^{1}$ & Shipment \#5 Load, Decon, and Leak Test & 05JणN36 & 280.00 & 6 \\
\hline PIM295 & $\frac{1}{K_{1}}$ & REC DOOT PM & TOJUN96" & 730.00 & 2 \\
\hline STM358 & $\frac{1}{A A_{1}}$ & Misc. Cask Loadunioad & T0JUN96 & 30.00 & $T$ \\
\hline BCT65 & $Q_{j}$ & Manipulator Changeouts & TगUस95 & 34.00 & $T$ \\
\hline SMTES & $P$ & Manipulator Changeouts & TTJUN95 & 10.00 & $T$ \\
\hline BCSP2070 & $\frac{1}{c \mid}$ & Shipment $\$ 5$ Prep, Load, and Leak Test Ċask & 13JUार96 & 280.00 & 4 \\
\hline$B C 726$ & $\frac{1}{0}$ & Manipulator Decon and Repair & T7JUN95 & 745.00 & $T$ \\
\hline BC5674 & $\mathrm{BB}$ & Moving Waste for NDA & T7JUN96 & 700.00 & $T$ \\
\hline $\sin 370$ & $A A_{1}$ & Misc. Cask Load/Unioad & 17JUN96 & 30.00 & $T$ \\
\hline SFIT3 & $x$ & Waste Disposal & 24JUN96 & 30.00 & $T$ \\
\hline $\sin 372$ & AA & Misc. Cask Loadrunload & $24 J 0 N 96$ & 30.00 & $T$ \\
\hline BCSP2080 & $c$ & Shipment \#6 Load, Decon, and Leak Test & 25JUN96 & 280.00 & 6 \\
\hline PN 18 & $\frac{1}{M 1}$ & Ship Consolidated SERF-FFTF Pins from 327 to & $25 J 01 N 96^{*}$ & 45.00 & $T$ \\
\hline OJJUE963 & & 1 & W & 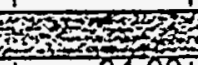 & \\
\hline BC168 & $\widetilde{Q}$ & Manipulator Changeouts & 01 Jutg6 & 34.00 & \\
\hline $8 \mathrm{BC2226}$ & Z) & TEPA A-Frame DOS & 01JUL96 & $1,020.00$ & $T$ \\
\hline De400415 & W & To-Cellcrane Prev. Maint. & 07J0[96*- & $-T, 825.00$ & $T$ \\
\hline
\end{tabular}




\begin{tabular}{|c|c|c|c|c|c|}
\hline $\begin{array}{l}\text { Activity } \\
\text { ID }\end{array}$ & $\begin{array}{l}\text { Est. } \\
\text { Typo }\end{array}$ & $\begin{array}{ccc} & \therefore & \text { Activity } \\
\therefore & \therefore & \text { Description }\end{array}$ & $\begin{array}{l}\text { Planned } \\
\text { Start }\end{array}$ & $\begin{array}{l}\text { Total .. } \\
\text { Dose }: .10\end{array}$ & $\begin{array}{l}\text { Original } \\
\text { duration }\end{array}$ \\
\hline SMT68 & $P \mid$ & Manipulator Changeouts & 07J0[96: & 10.00 & 1 \\
\hline $5 \pi 374$ & AAI & Misc. Cask Load/Unload & 01JUL96 & 30.00 & 3 \\
\hline BCSP2085 & & Shipment \#6 Prep, Load, andLeak Test Cask & 03JUL96 & 280.00 & 4 \\
\hline BCT98 & & Waste Compactiontuecon & 08JUL96 & 10.00 & $T$ \\
\hline SM376 & $A A_{1}^{1}$ & Misc. Cask Loadunioad & 08JUL96 & 30.00 & $T$ \\
\hline$B C 725$ & $\sigma_{j}^{1}$ & Manipulator Decon and Repair & 72JU[96 & 745.00 & $T$ \\
\hline BCE67.5 & $\mathrm{BB}$ & Moving Waste for NDA & 16JUL96 & 700.00 & $T$ \\
\hline BCSP2095 & & Shipment \#7 Load, Decon, and Leak Test & 17JUL96 & 280.00 & 6 \\
\hline $\sin 378$ & $\overline{A A}_{i}$ & Misc. Cask Load/UnIoad & 17JUL96 & 30.00 & $?$ \\
\hline$B C 770$ & $Q$ & Manipulator Changeouts & 18JUL96 & 34.001 & 9 \\
\hline STM170 & & Manipulator Changeouts. & 18JUL96 & 70.00 & $T$ \\
\hline PM264 & & A-Frames & $20 J 0\left[96^{\circ}\right.$ & $95.00_{i}^{1}$ & $T$ \\
\hline STM380 & AA & Misc. Cask Load/Unioad & 24JUL95 & $30.00 \vdots$ & $T$ \\
\hline $8 C 50032$ & $C_{1}$ & Remove Ainock Service Jumpers - TA & 25JUL95 & 420.00 & 4 \\
\hline BCSP2100 & & Shipment \#7Prep, Load, and Leak Test Cask & $25 J 0 L 95$ & 280.00 & 4 \\
\hline SFT14 & & Waste Disposal & 25JUL96" & 30.00 & 1 \\
\hline SM382 & $A A_{1}$ & Misc. Cask Load/Unioad & $30 J 0 L 95$ & 30.00 & T \\
\hline $\mathrm{BCT} 72$ & $Q_{i}$ & Manipulator Changeouts & उगJणL95 & 34.00 & T \\
\hline SIMt72 & $P_{1}$ & Manipulator Changeouts & 31गUL96 & 70.001 & $T$ \\
\hline 01AUG96 & 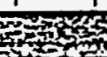 & 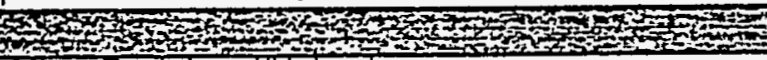 & 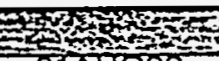 & 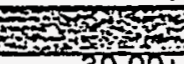 & 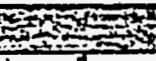 \\
\hline sin384 & $A A_{1}$ & Misc. Cask Load/Unload & $01 A \cup \operatorname{GOS}$ & 30.00 & \\
\hline$B C 150050$ & $D_{1}$ & Decontaminate 6-Ton Crane & 05AUG96 & $2,380.00$ & 5 \\
\hline $8 \mathrm{C} 200$ & $R_{1}$ & Waste Compaction/Decon & 05AUG96 & 70.00 & $T$ \\
\hline STM386 & $A A_{1}$ & Misc. Cask Loadunioad & OTAUG95 & 30.00 & $T$ \\
\hline$B C 28195$ & $s_{1}$ & RMTIPOG Filer Changeout & O8AUG95" & 170.00 & $T$ \\
\hline BC600230 & $\frac{1}{A !}$ & Spray Down Coverblocks \& Reinstall & 0ல8AंG95 & 420.00 & 2 \\
\hline Sc400415 & $\frac{1}{1}$ & S-Cell Par Prev. Maint. & O8AUG95 & $2,520.00$ & $T$ \\
\hline PN 24 & mi & Ship C-I Shipment to FFTF. & O9AUG9! & 45.00 & 5 \\
\hline PN 25 & $m_{1}$ & ShIP MFA-1TMFA-2IACO-3 FuelPInS tO FFTF & O9RUG96 & 45.00 & 5 \\
\hline PN 26 & $m$ & Ship PNCWaste (SERF CंellFuel PIns) to FFTF & OOAUG96:- & 45.00 & 5 \\
\hline$B C 150055$ & G) & Remove 6-Ton Crane & T2AUG95 & T,330.00 & 70 \\
\hline BC127 & $\sigma_{1}^{1}$ & Manipulator Decon and Repair & T5AUG96 & 745.00 & $T$ \\
\hline BC6576 & $\mathrm{BB}$ & Moving Waste for NDA & T9AUG96 & 700.00 & $T$ \\
\hline BC174 & Q) & Manipulator Changeouts & 20AUG96 & 34.00 & $T$ \\
\hline $\sin 174$ & $P$ & Manipulator Changeouts & 20AUG96 & 10.00 & $T$ \\
\hline$B C 150060$ & & Install To-Ton Capacify Crane & 26AUG96 & $7,330.00$ & 10 \\
\hline$B C 2228$ & Z & HEPA A-Frame DOS & उOAUG96 & $1,020.00$ & 7 \\
\hline SFT15 & $x$ & Waste Disposal & 30AUG96" & 30.00 & T \\
\hline 01SEP96 & 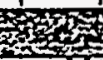 & 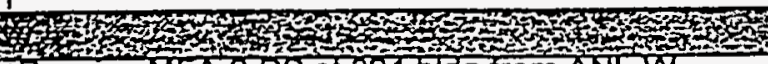 & & & 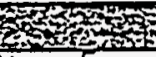 \\
\hline PN 27 & M & Receive MFA-2 DS at 324 bidg from ANL-W & 03 SEP96 & 45.00 & 5 \\
\hline $\sin 388$ & $A A$ & Misc. Cask Load/Unioad & O3SEPQ6 & 30.00 & 7 \\
\hline
\end{tabular}




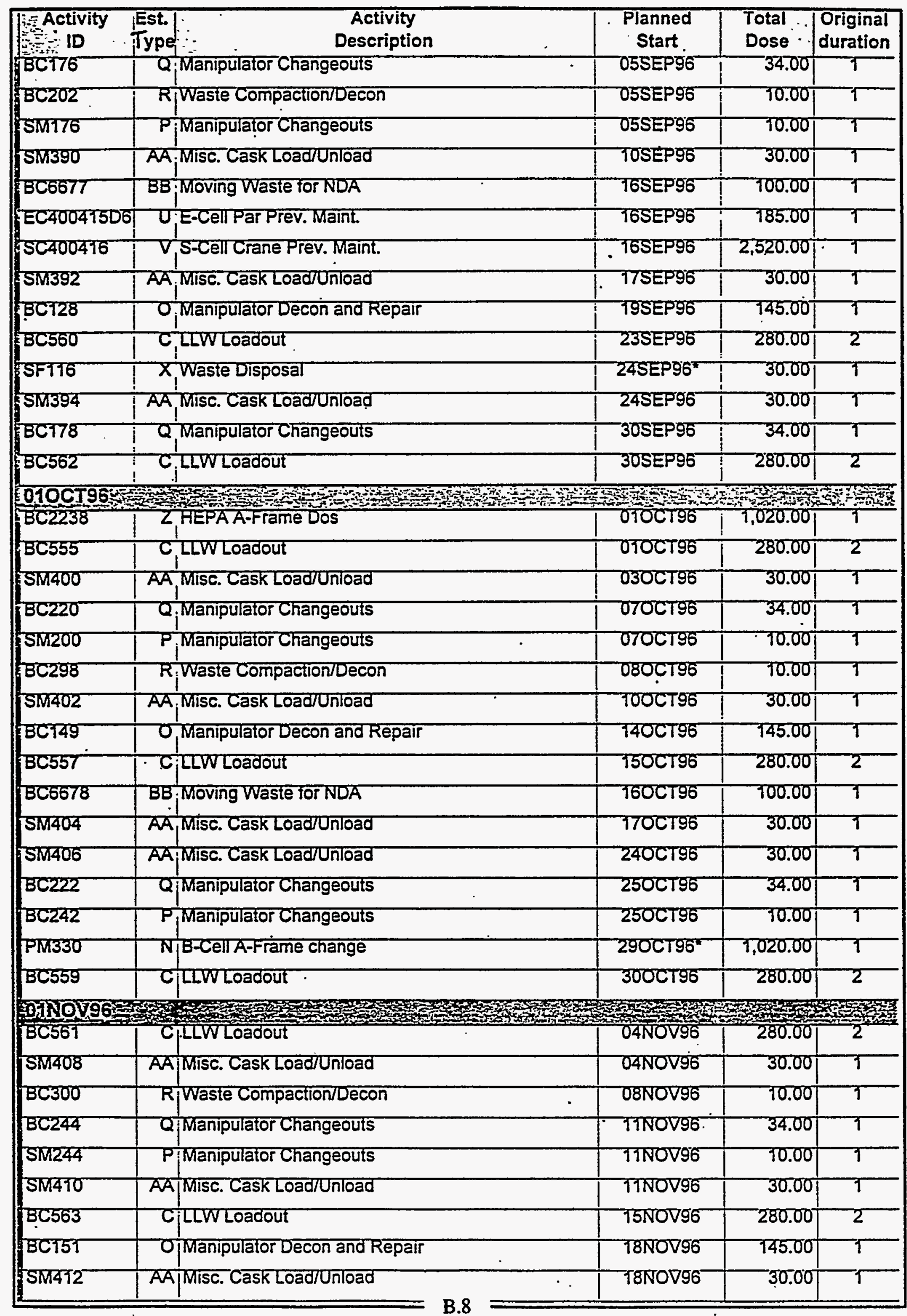




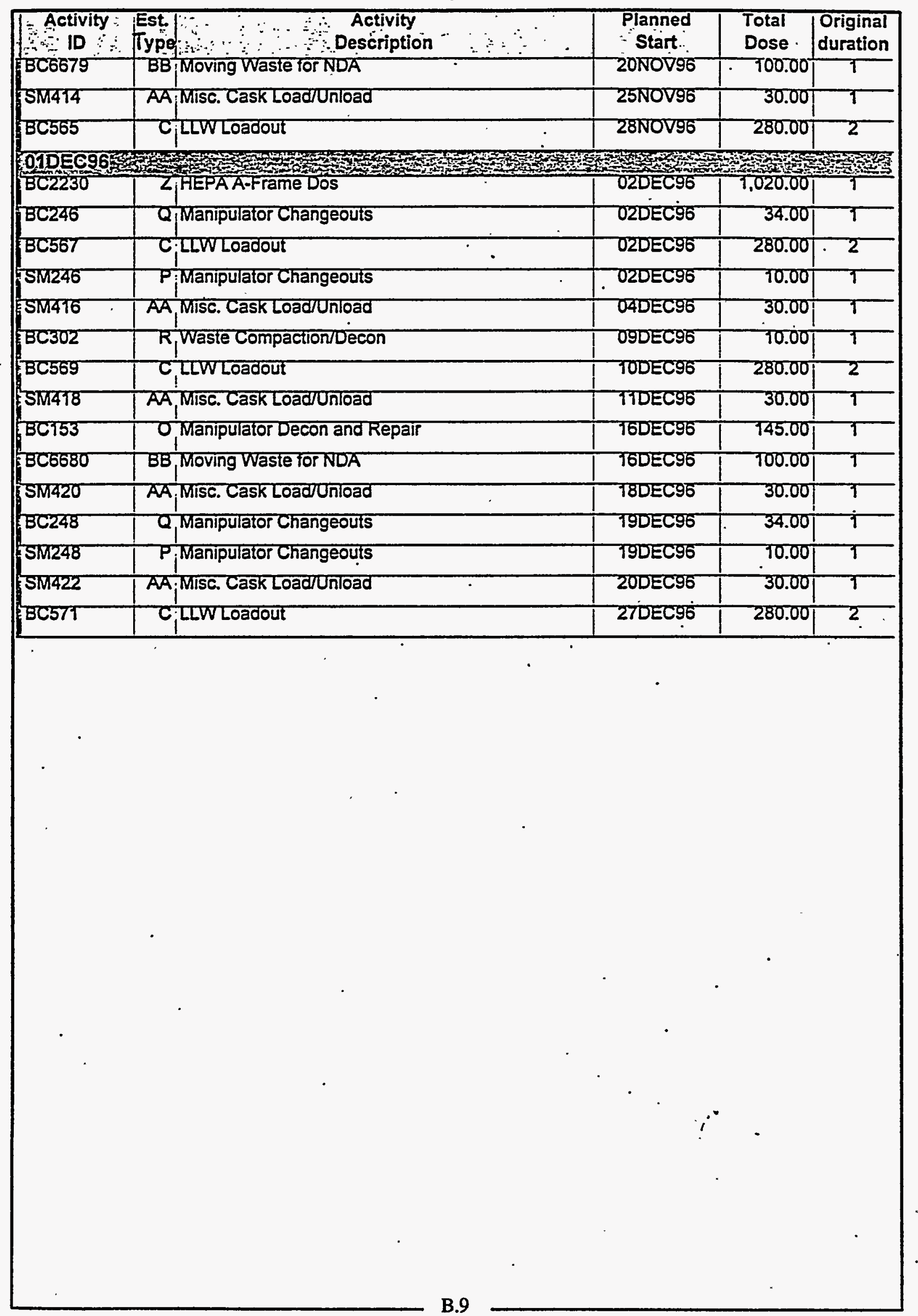




\section{RESOURCE}

\section{LOADING}

\section{SUMMARIES}

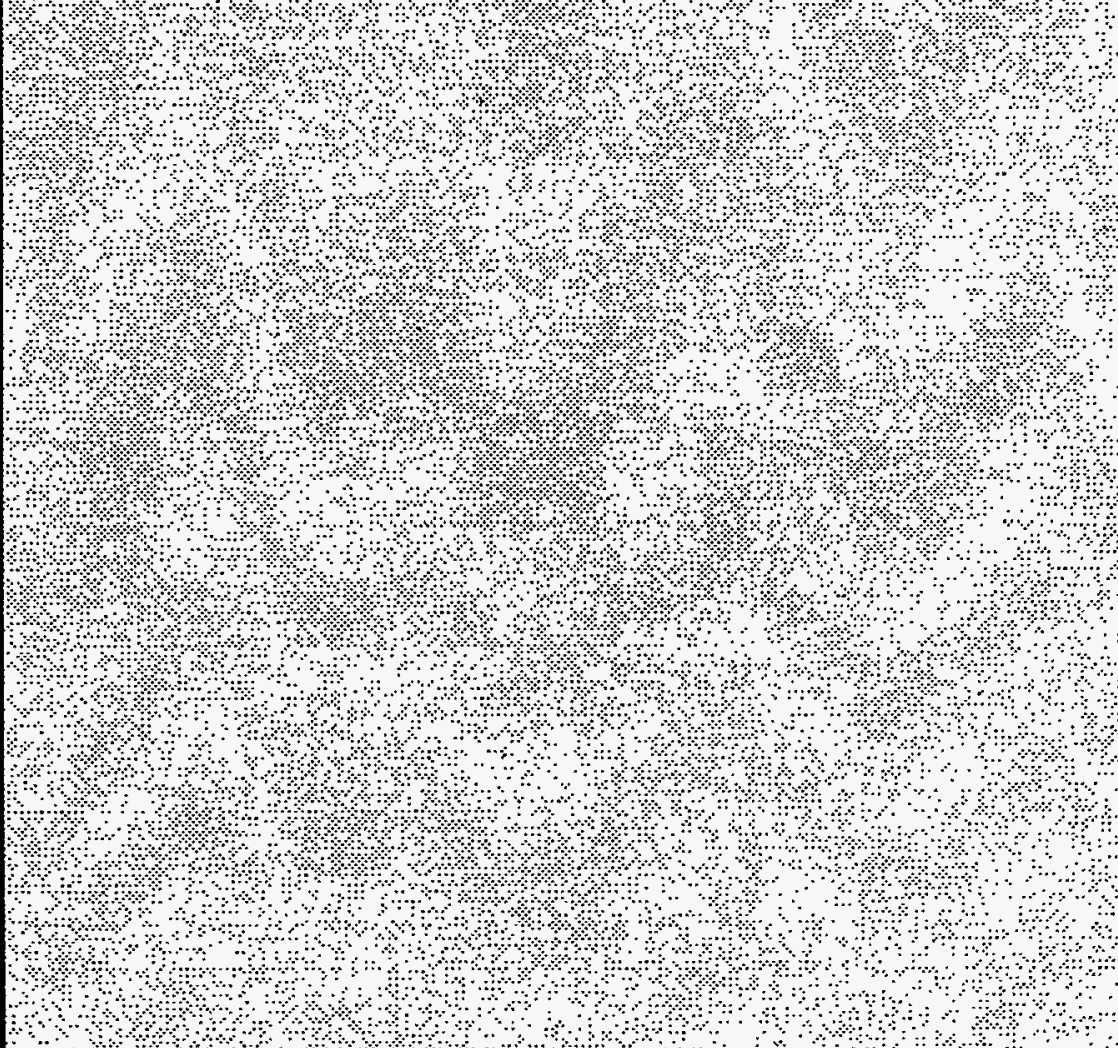


REPORT. DATE $\begin{gathered}\text { I0JAN96 RUN NO. } 208 \\ 13: 23\end{gathered}$

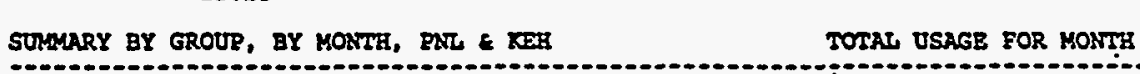

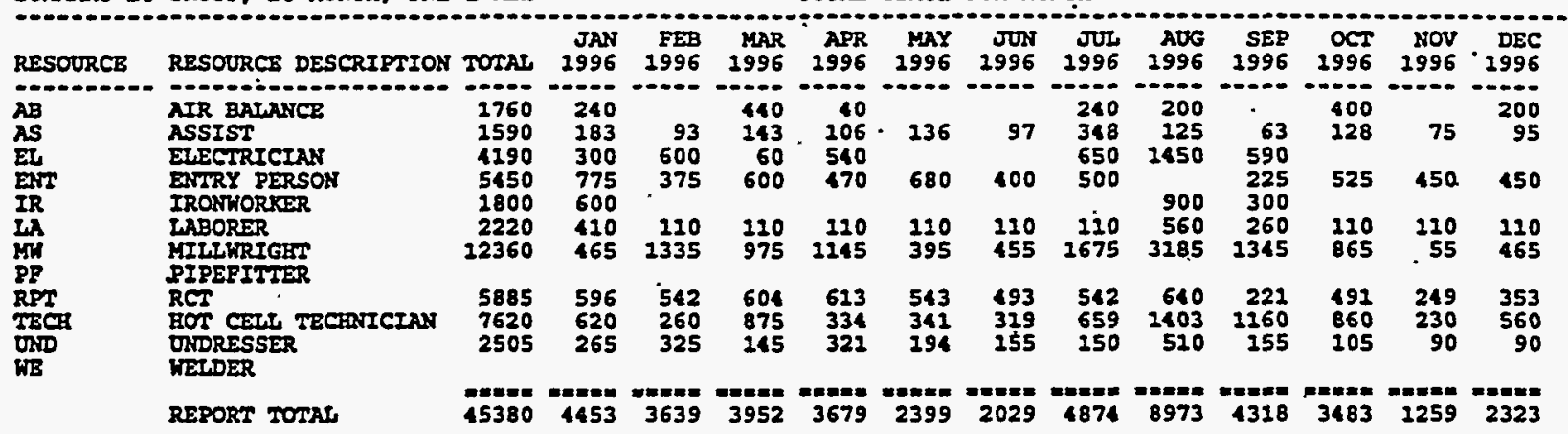

START DATS OIJAN95 $F$ DATA DATE 01JAN9S $P$ PRINAVERA PROJECT PLANONER

RESOURCE LOADING REPORT . 
PRIMAVERA PROTECT PLANNER

REPORT DATE 10JAN96 RON NO. 209

RESOURCE LOADING REPORT

START DATE 01JAN95

$13: 26$

TOTAL USAGE FOR MONTH

DATA DATE 02JAN95 $p$

STMNARY BY GROUP, BY MONTE, KJH ONLY

JAN FEB MAR APR MAY JUN JU ADG SEP OCT NOV

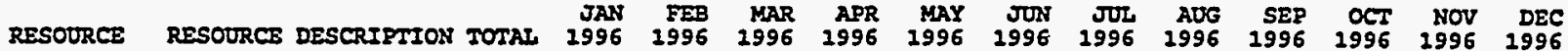

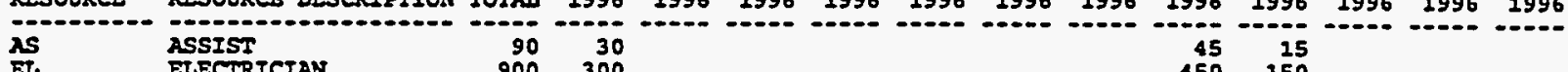

ELECIRICIAN

IRONKORKER

LABORER

$\begin{array}{ll}\text { IR } & \text { IABORER } \\ \text { PF } & \text { PIPEFITIER } \\ \text { UND } & \text { UNDRESSER } \\ \text { WE } & \text { WETDER }\end{array}$

$\begin{array}{ll}\text { IR } & \text { IABORER } \\ \text { PF } & \text { PIPEFITIER } \\ \text { UND } & \text { UNDRESSER } \\ \text { WE } & \text { WETDER }\end{array}$

$\begin{array}{ll}\text { IR } & \text { IABORER } \\ \text { PF } & \text { PIPEFITIER } \\ \text { UND } & \text { UNDRESSER } \\ \text { WE } & \text { WETDER }\end{array}$

$1800 \quad 600$

2020.310

$10 \quad 10$

REPORT TOTAL

$300 \quad 100$

900300

$10 \quad 20 \quad 20$

$=010=0.040$

$150 \quad 50$

$4110 \quad 1340 \quad$ $10 \quad 10 \quad 10 \quad 10 \quad 10$

$10 \quad 2005 \quad 675$

$=-\frac{10}{10} \quad 10=20$ 


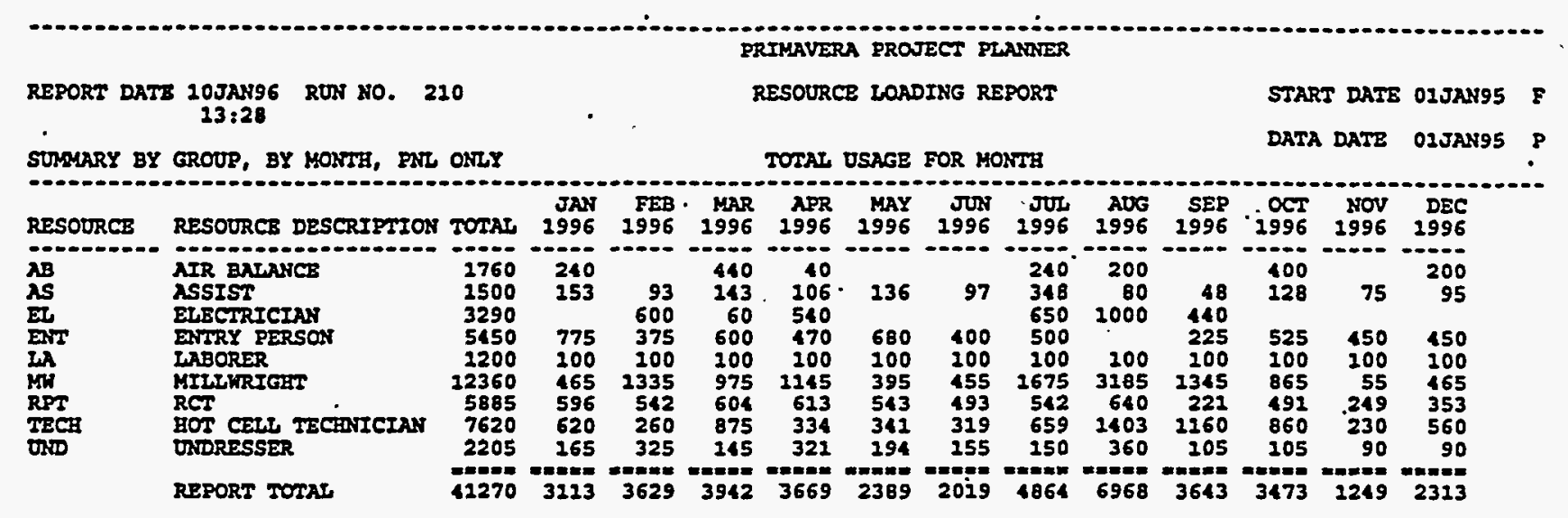




\title{
RESOURCE
}

\section{LOADING}

\author{
DETAIL
}

BY ACTIVITY

PNNL

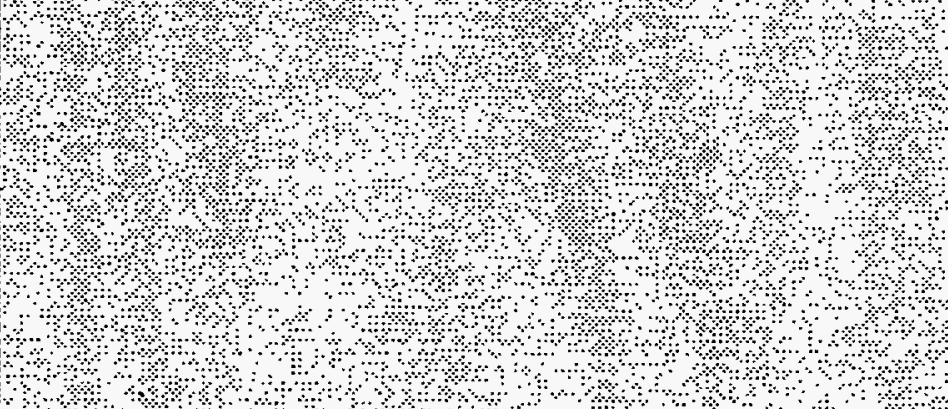

B. 14 


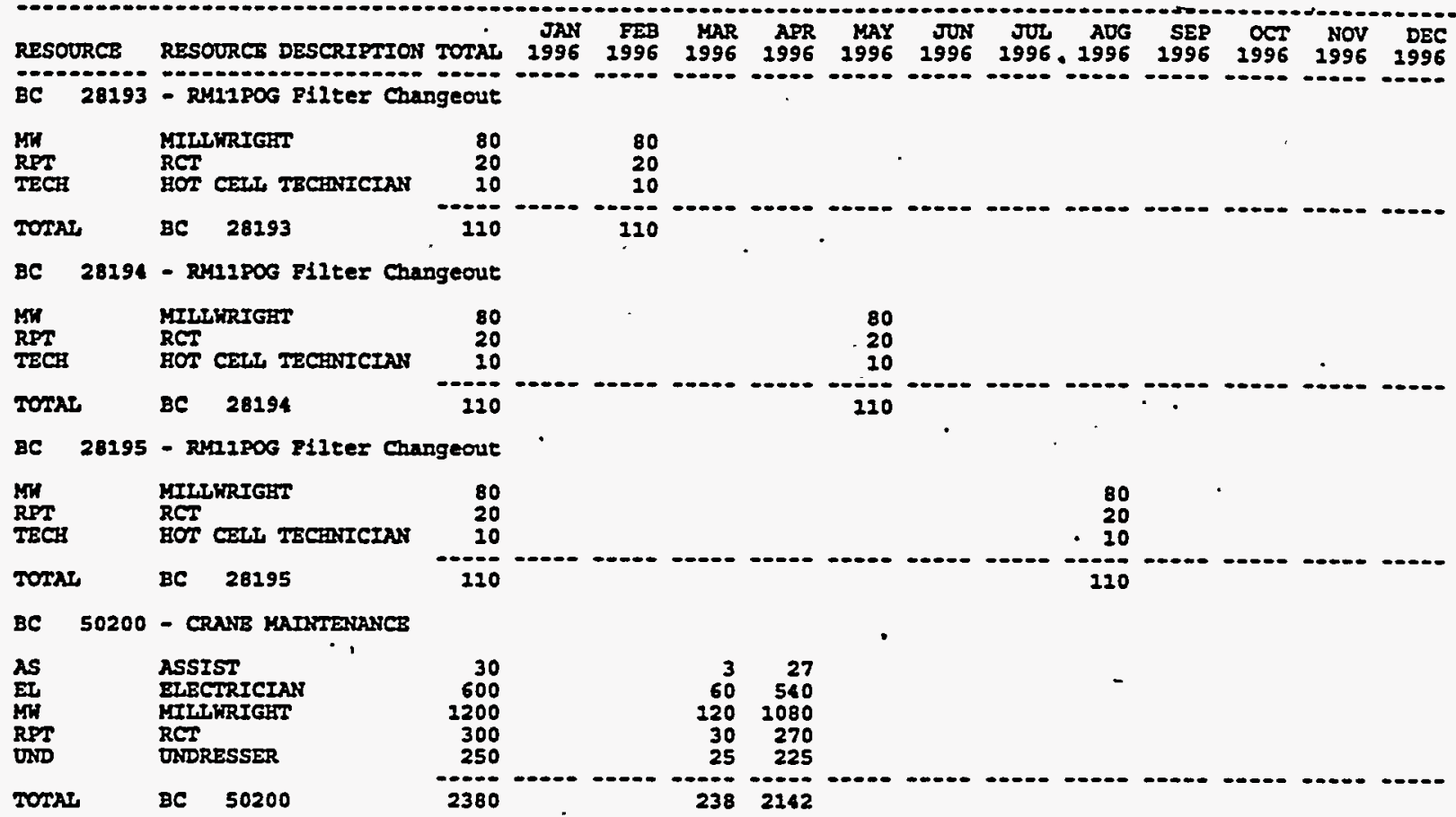

TOTAT BC 50200

BC 60031 - HASTB CAMPAIGH 6 (SEP95)

$\begin{array}{ll}\text { AS } & \text { MSSIST } \\ \text { ENT } & \text { ENTRY DERSON } \\ \text { RPT } & \text { RCI } \\ \text { OND } & \text { UNDRESSER }\end{array}$

TOTAI . BC 60031

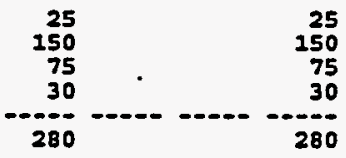

BC 90040 - 5th CRANE MAINTENANCE -FY96

$\begin{array}{ll}\text { AS } & \text { ASSIST } \\ \text { EL } & \text { ELECIRICIAN } \\ \text { MU } & \text { MILLIRIGHT } \\ \text { RPT } & \text { RCT } \\ \text { UND } & \text { UNDRESSER } \\ \text { TOTAT } & \text { BC } \quad 90040\end{array}$

\begin{tabular}{rr}
30 & 30 \\
600 & 600 \\
1200 & 1200 \\
300 & 300 \\
250 & 250 \\
\hline 2380 & 2380
\end{tabular}

BC 150050 - Decontaminate 6-Ton Crane

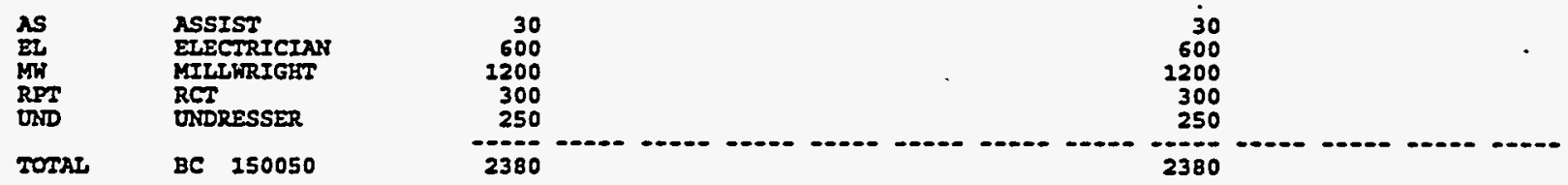

BC 600230 - SPRAY DOLA COVER BLOCRS A REINSTALI

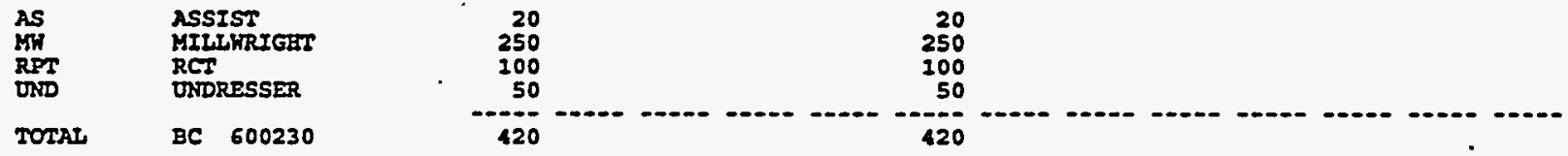




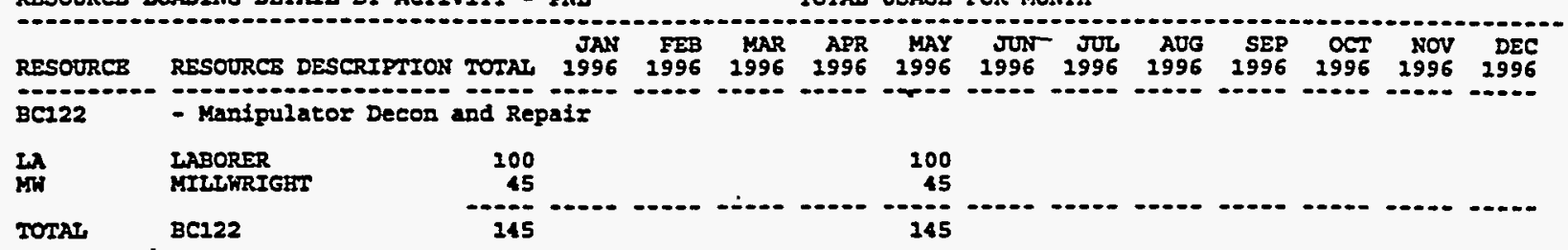

BC125 - Manipulator Decon and Repair

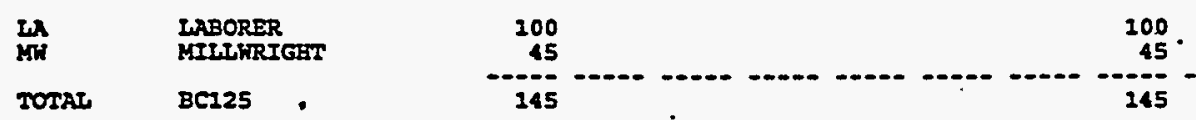

BC126 - Manipulator Decon and Repair

LA LABORER

TOTAS BC126
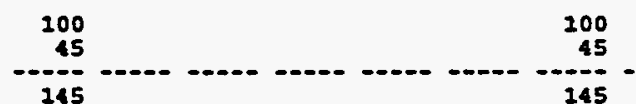

BC127 - Maripulator Decon and Repair

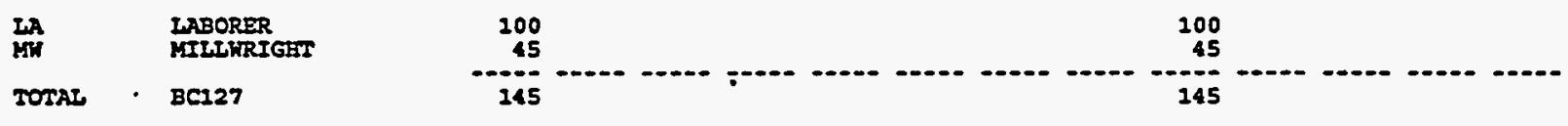

BC128 - Manipulator Decon and Repair

LA LABORER

MT MILWRIG

TOTAL BC228

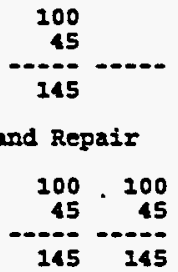

Mnt

- Manipulator Decon and Repair

BC232

IABORER

MILLWRIGET

TOTAI BC13I

$$
145145
$$

BC132 - Manipulator Decon and Repair

LA LABORER

TOTAL BC132

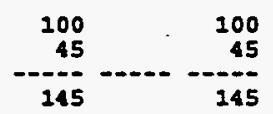

BC133 - Manipulator Decon and Repair

IA LABORER

MH - MILIKRIGHT

TOTAL BC133

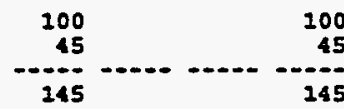

BC134 - Manipulator Decon and Repais

LA LABORER

MW MILLWRIGHI

TOTAL BC134

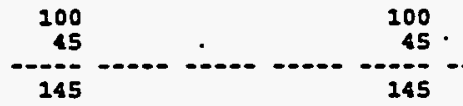

BC149 - Manipulator Decon and Repair

LA IABORER

MW MILLRIGET

TOTAL BC149

100
45
145

100
45
145 


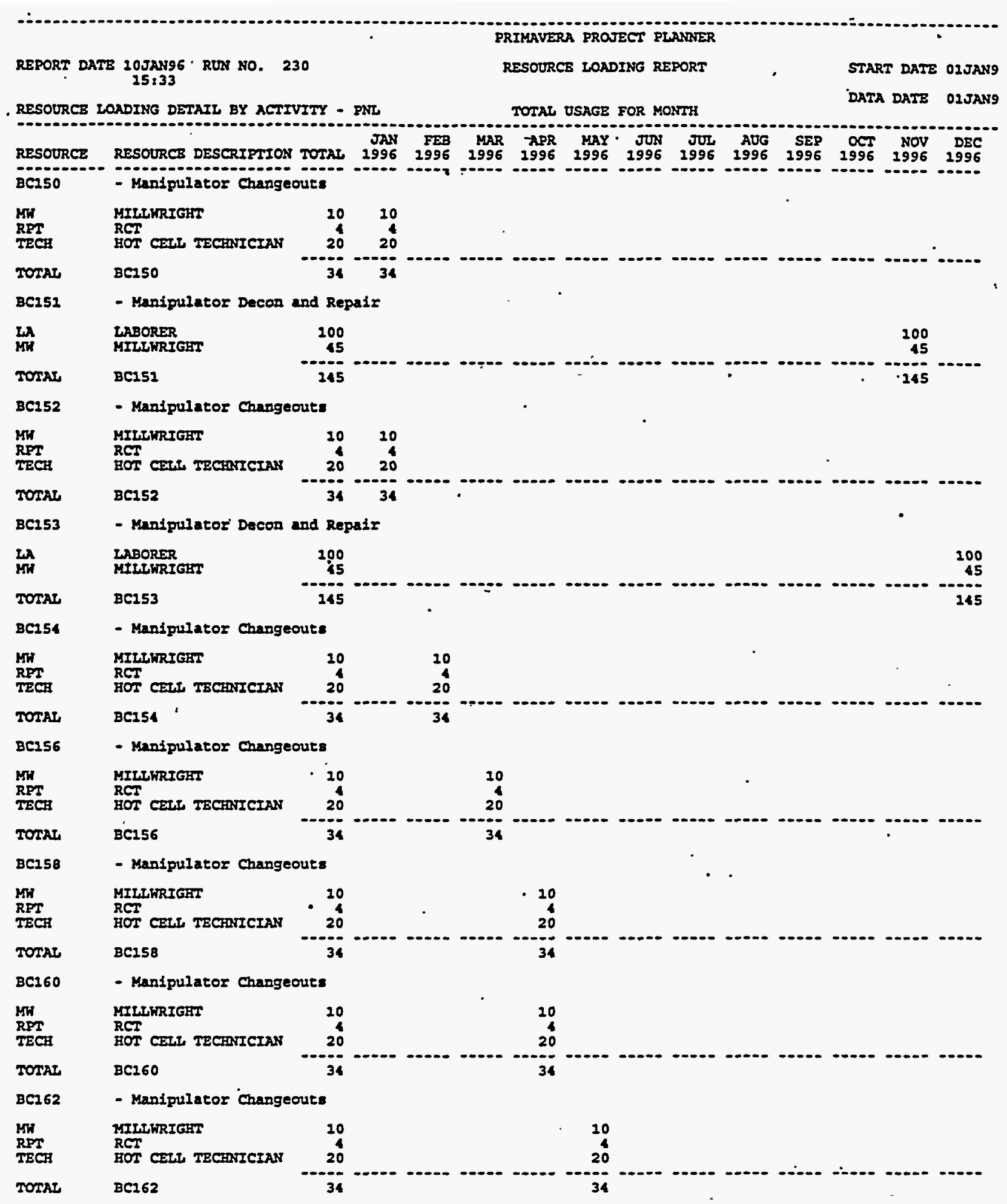

B.17. 
RESOURCE LOADING DETAIL BY ACTIVITY - PNL

TOTAL USAGE FOR MONTH

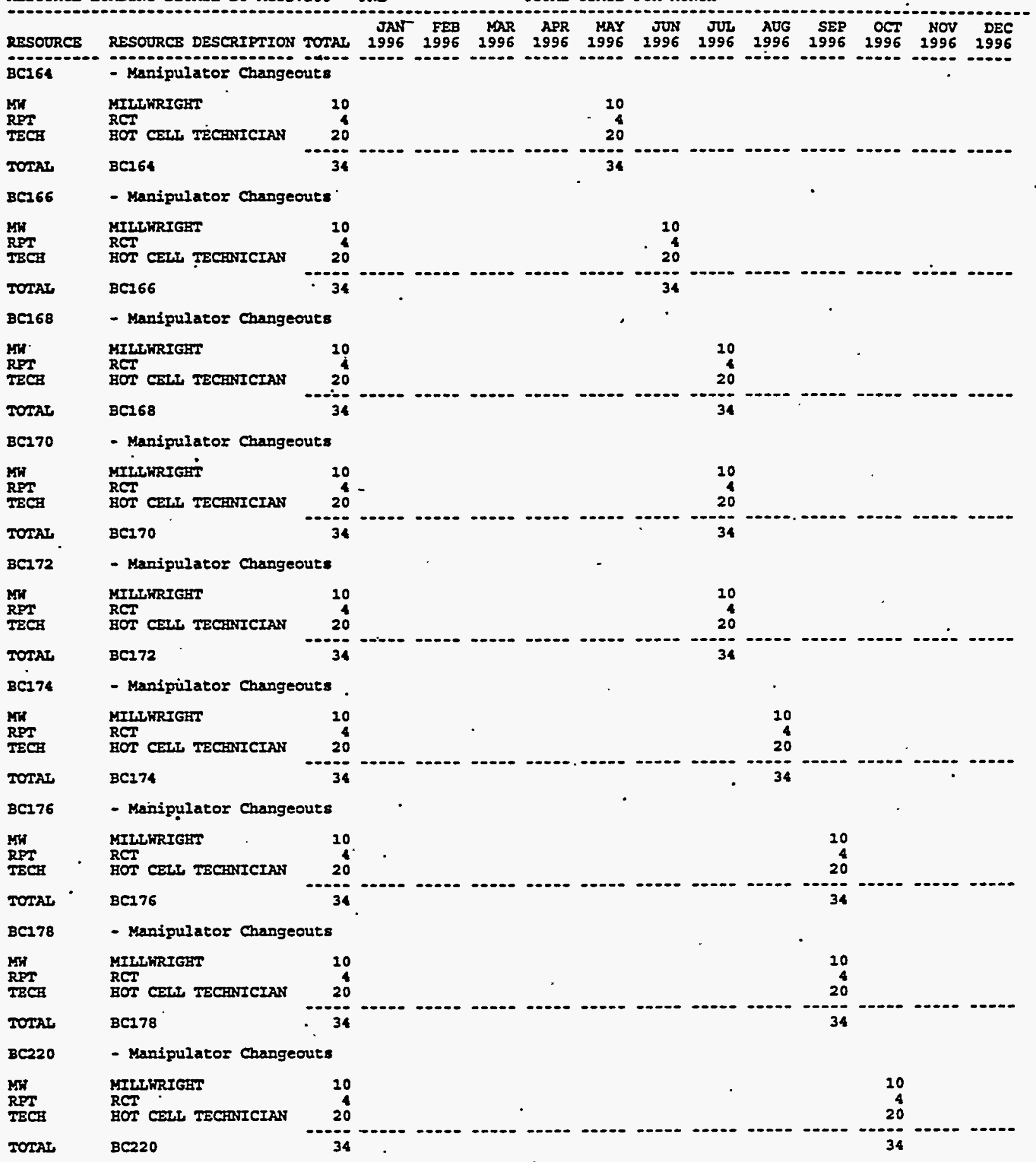


RESOURCE LOADING DETAIL BY ACTIVITY - PRL

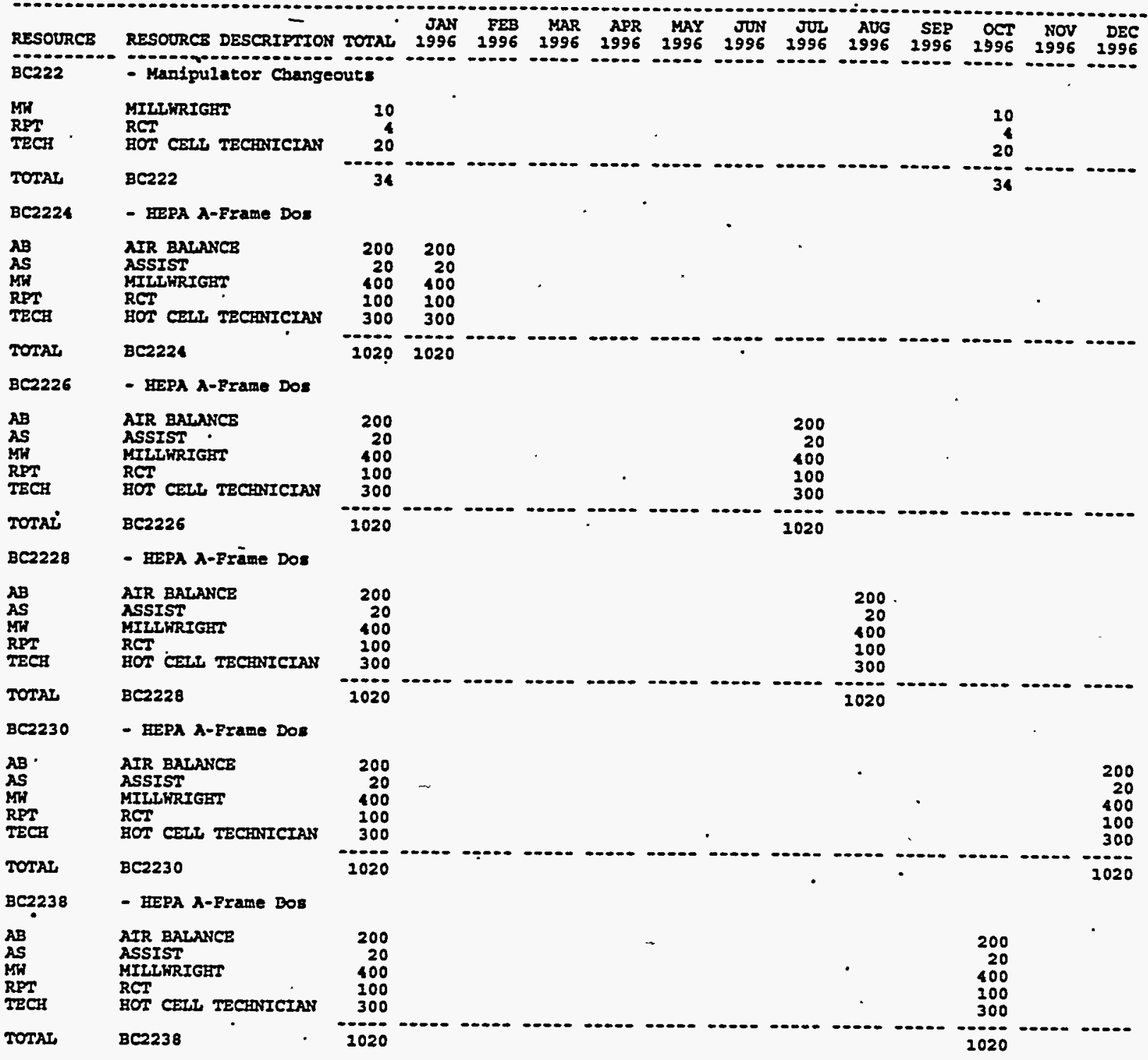

BC242 - Manipulator Changeouts

TECH HOT CELL TECHICIAN

TOTAL $\mathrm{BC2} 22$

BC244 - Manipulator Changeouts

MH MILLWRIGHT

RDT

RCT

TECH

ROT CELI TECHNICIAN

$\begin{array}{r}10 \\ 4 \\ 20 \\ \hline 34\end{array}$

TOTAL BC244

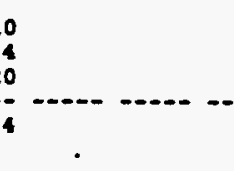


PRIMAVERA PROJECT PLANNER

REPORT DATE I0JAN96 RUN NO. 230

START DATE 02JAN9

$$
15: 33
$$

TOTAL USAGE FOR MONTH

RESOURCE LOADING DETAIL BY ACTIVITY - PNL

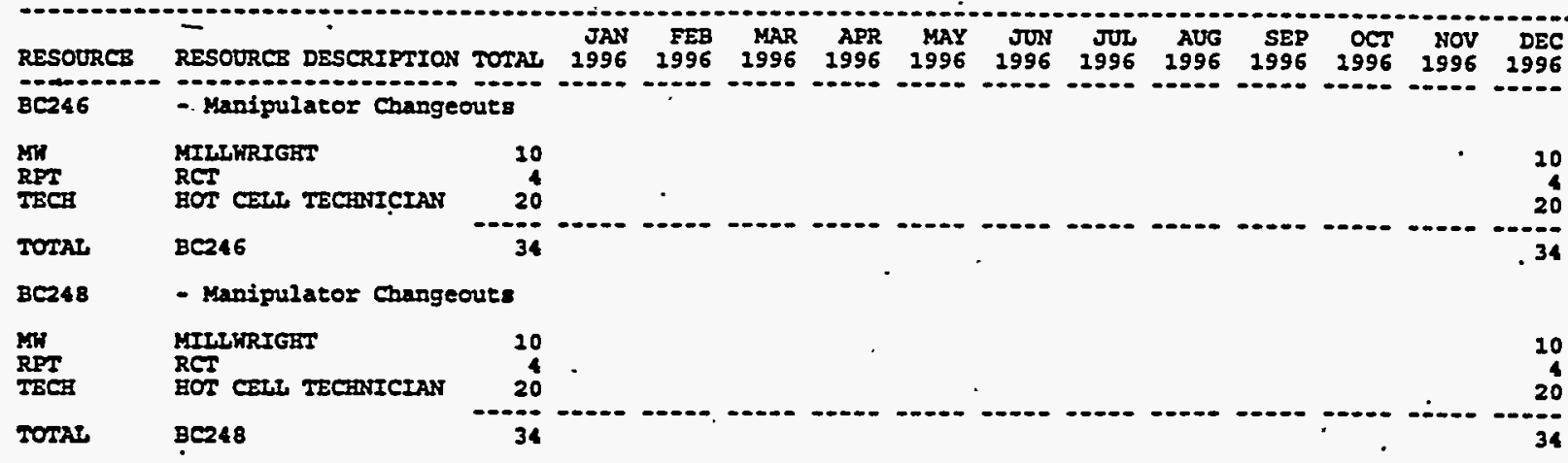

BC50032 - Remove Airlock Service Jumpers - $1 \mathrm{~A}$

$\begin{array}{ll}\text { AS } & \text { ASSIST } \\ \text { EL } & \text { ELECTRICIAN } \\ \text { RPT } & \text { RCT }\end{array}$

UND. UNDRESSER

TOTAL BC50032

BC555 - IIN IOadout

AS - ASSIST

RPS RCT

UND UTDRESSER

Total BC555

BC557 - - IIN Loadout

As ASSISI

DNT ASSIST

$\begin{array}{ll}\text { RPT } & \text { RCI } \\ \text { UND } & \text { ONDRESSER }\end{array}$

IOTAL BC557

BC559 - IIN IOadout

AS ASSIST

ENT RPT ENSIST PERSON

UND UNDRESSER

TOTAI BC559

BC560 - ILW Ioadout

AS ASSIST

ETI - ENTRY PERSON

UW

TOTAI BC560

BC561 - IfW Loadout

AS ASSIST

ENT ENTRY PERSON

RPT RCT

UND UNDRESSER

TOTAI BC561
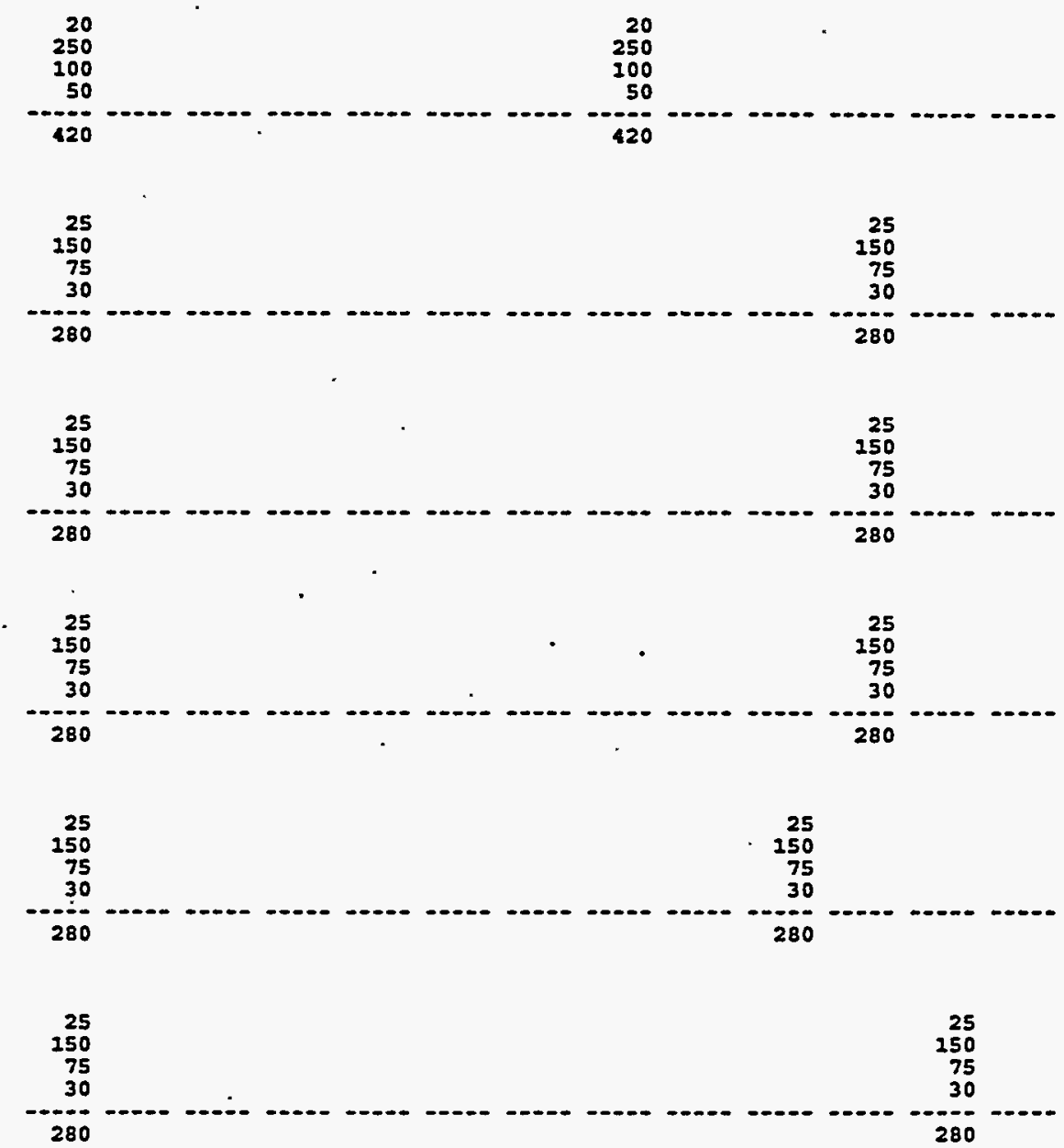
RESOURCE RESOURCB DESCRTPTION JOA JAN MAR APR MAY JUN JUL AUG SEP OCT NOV DEC $\begin{array}{lllllllllll} & 1969 & 1996 & 1996 & 1996 & 1996 & 1996 & 1996 & 1996 & 1996 & 1996\end{array}$

BC562 - LLY LOadout

AS ASSIST

ENT

RPT RCT

UNDRESSER

TOTAL BC562

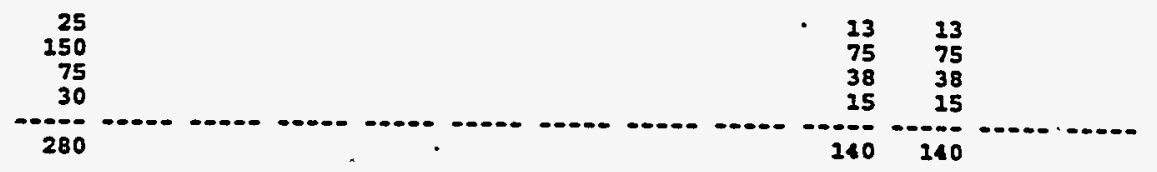

BC563 - LEV Loadout

AS ASSIST

ENT ENTRY PERSON

RPT RCT

UND ONDRESSER

TOTAL BC563

BC565 - LLK LoAdout

\section{AS ASSIST}

ENTT ENTRY PERSON

UND RCT

TOTAL BC565

BC567 - LLW LOAdout

$\begin{array}{ll}\text { AS } & \text { ASSIST } \\ \text { ENT } & \text { ENIRY PERSON } \\ \text { RPT } & \text { RCT }\end{array}$

UND UNDRESSER

TOTAL BC567

BC569 - LLW LOAdOUE

AS ASSIST

ENT

UND RTD RTDRESSER

TOTAL BC569

BC57I - LLW LOAdOUE

AS ASSIST

ENT

UND UNDRESSER

TOTAL BC571

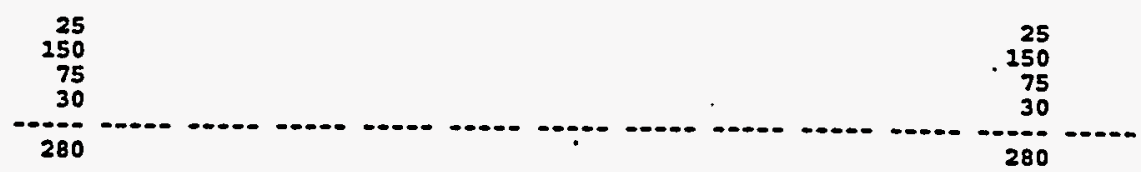

BC600230

- Spray Down Coverblocks Reinstall

$\begin{array}{ll}\text { AS } & \text { ASSIST } \\ \text { MHT } & \text { MILLTRIGTT } \\ \text { RPT } & \text { RCT } \\ \text { UND } & \text { UNDRESSER }\end{array}$

TOTAL BC600230

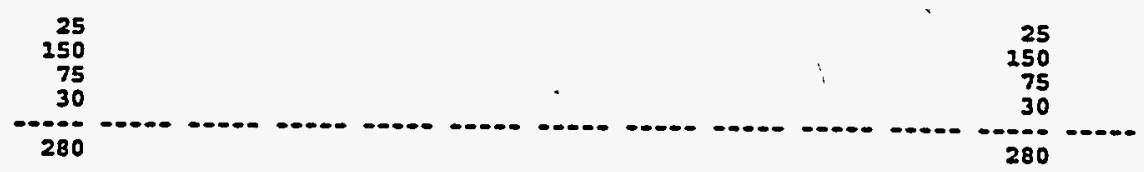

BC6669 - Mowing Maste for NDA

TECH HOT CELL TECHNTCIAN $100 \quad 200$

TOTAL BC6669 $100 \quad 100$

BC6670 - Moving Waste for MDA

TECH

.HOT CELL TECHNICIAN 100

100 


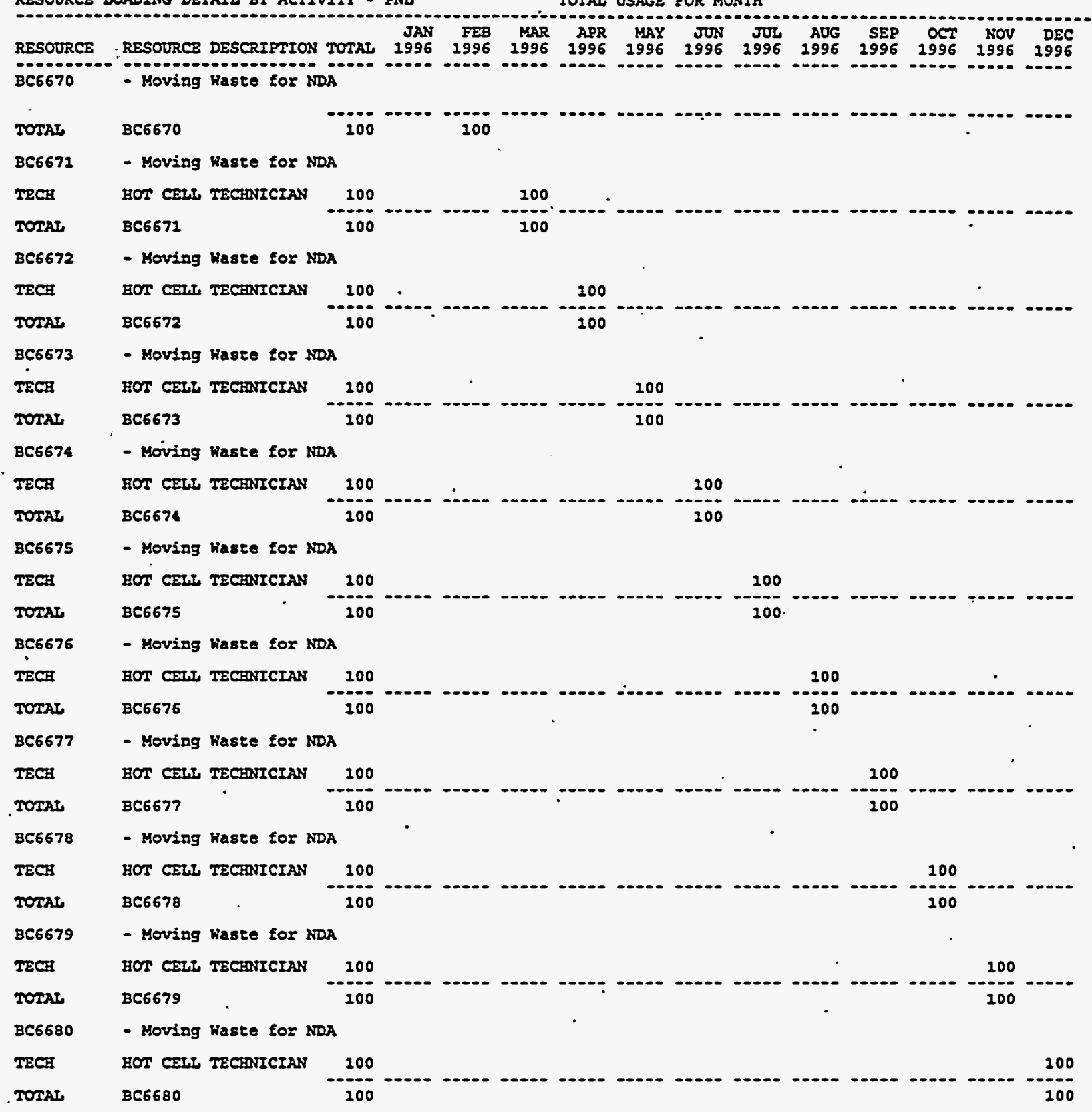

BCD313 - Loadout 3rd G.C. REFR Brick in Airlock

$\begin{array}{llrr}\text { AS } & \text { ASSIST } & 25 & 25 \\ \text { ENT } & \text { ENIRY PERSON } & 150 & 150 \\ \text { RPT } & \text { RCT } & 75 & 75 \\ \text { UND } & \text { UNDRESSER } & 30 & 30\end{array}$




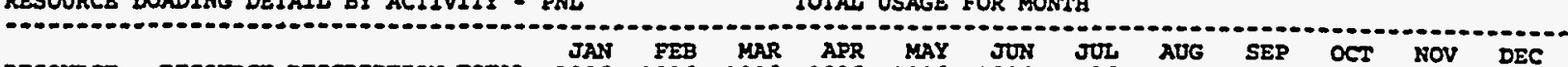

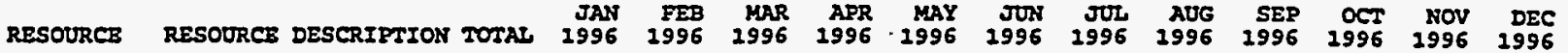

G.

BCD313 - LOadout 3rd G.C. REER Brick in Airlock

TOTAI BCD313

BCO323 - SEG Casak Shipoent / Liner * ??

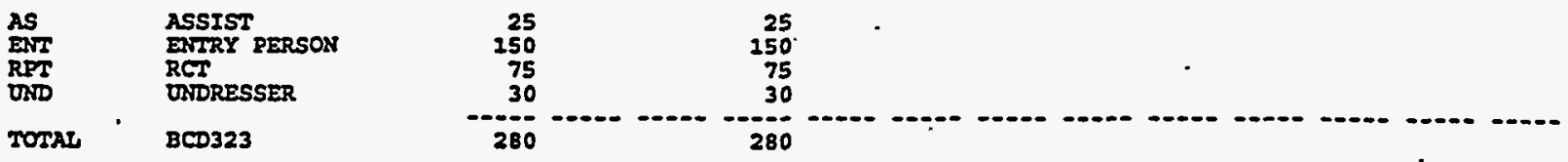

BCD327 - SEG Cask Shipment /Liner 37

$\begin{array}{ll}\text { AS } & \text { ASSIST } \\ \text { ENT } & \text { ENTRY PERSON } \\ \text { RPT } & \text { RCT }\end{array}$

OND UNDRESSER

TOTAL BCD327

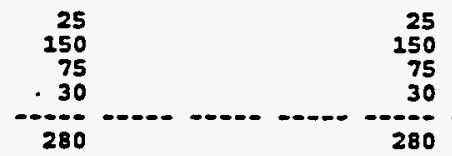

BCD405 - Loadout of Melter Glass in Airlock

AS ASSIST

ENIRY PERSON $25 \quad 25$

$\begin{array}{ll}\text { RPT } & \text { RCT } \\ \text { UND } & \text { UNDESSER }\end{array}$

TOTAL - BCOE05

\begin{tabular}{rr}
250 & 25 \\
75 & 75 \\
30 & 30 \\
\hline 280 & 280
\end{tabular}

BCPX1711 - Loadt of MItr Feed/Lig Mtl Seal/Oil Abs in Airlk

$\begin{array}{ll}\text { AS } & \text { ASSIST } \\ \text { ENT } & \text { ENIRY PERSON } \\ \text { RPT } & \text { RTT } \\ \text { UND } & \text { UNDRESSER } \\ \text { UNT } & \end{array}$

TOTAL BC'PX4711

\begin{tabular}{rr}
25 & 25 \\
250 & 150 \\
75 & 75 \\
30 & 30 \\
\hdashline 280 & 280
\end{tabular}

BCPX4716 - 1st Loadout of High Dose Metals in Airlock (scw)

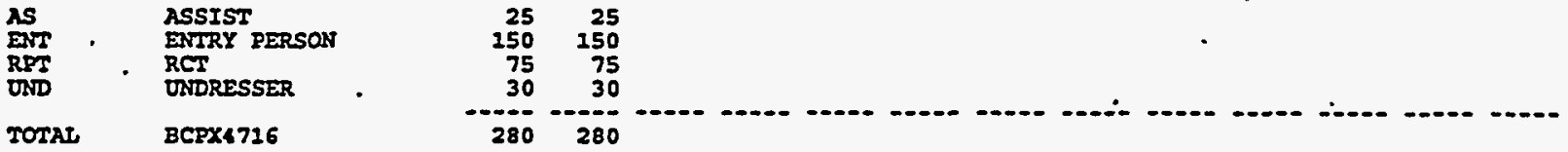

BCPX4721 - Ist Loadout of Dispersible in Airlock

$\begin{array}{ll}\text { AS } & \text { ASSIST } \\ \text { ENT } & \text { ENTRY PERSON } \\ \text { RPT } & \text { RCT } \\ \text { UND } & \text { UNDRESSER }\end{array}$

TOTAS BCPX4721

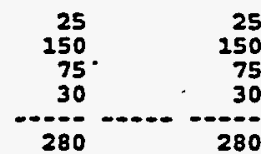

BCPX4736 - 2nd Loadout of Dispersible in Airlock

$\begin{array}{ll}\text { AS } & \text { ASSIST } \\ \text { ENT } & \text { ENTRY PERSON } \\ \text { RPT } & \text { RCT }\end{array}$

$\begin{array}{ll}\text { RPT } & \text { RCT } \\ \text { OND } & \text { ONDRESSER }\end{array}$

TOTAL BCPX4736

\begin{tabular}{rr}
25 & 25 \\
150 & 150 \\
75 & 75 \\
30 & 30 \\
\hline 280 & 28
\end{tabular}

BCPX4741 - 2st Loudout of Refractory in Airlock

AS

$$
\text { ASSIST }
$$

ENTRY PERSON

$\begin{array}{rrr}25 & 13 & 13 \\ 150 & 75 & 75\end{array}$




DESOURE TEB MAR APR MAY JUN JUL AUG SEP OCT NOV DEC

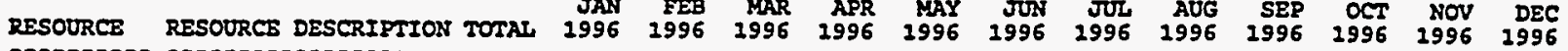

BCPX4741 - $18 \mathrm{E}$ loadout of Refractory in Adrlock

RPT RCT $\quad 75 \quad .38 \quad 38$

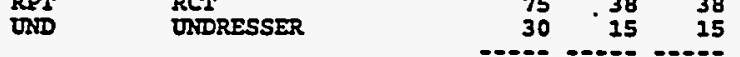

TOTAL BCPX4741 $280 \quad 140 \quad 140$

BCSP2000 - Shipment \#1 Load and Decon Overpacks

$\begin{array}{ll}\text { AS } & \text { ASSIST } \\ \text { ENT } & \text { ENTRY PERSON } \\ \text { RPT } & \text { RCT } \\ \text { DND } & \text { DNDRESSER }\end{array}$

TOTAI - BCSP2000

25
150
75 $\quad \begin{array}{r}25 \\ 350 \\ 30\end{array} \quad \begin{array}{r}75 \\ 30 \\ \hline 280\end{array}$

BCSP2010 - Shipment 11 Prep, Load, and Leak Test Cask

$\begin{array}{ll}\text { AS } & \text { ASSIST } \\ \text { ENT } & \text { ENTIRY PERSON } \\ \text { RFT } & \text { RCT } \\ \text { UND } & \text { UNDRESSER }\end{array}$

UND UNDRESSER

TOTAL BCSP2010

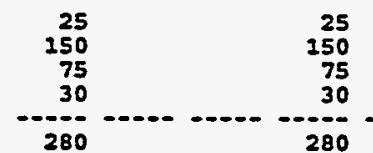

BCSP2020 - Shipment 22 Load, Decon, and Leak Test Overpacks

AS ASSIST

ERT

$\begin{array}{ll}\text { RPT } & \text { RCT } \\ \text { UND } & \text { UNDRESSER }\end{array}$

TOTAL BCSP2020

BCSP2025 - Shipmest 2 Prep, Load, and Leak Test Cask

$\begin{array}{ll}\text { AS } & \text { ASSIST } \\ \text { ENT } & \text { ENTRY PERSON } \\ \text { RPT } & \text { RCT } \\ \text { UND } & \text { UNDRESSER }\end{array}$

UND UNDRESSER

TOTAL BCSP2025

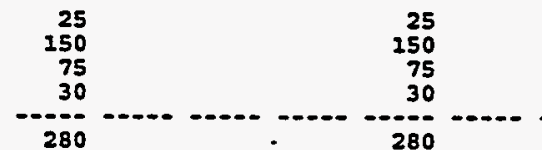

BCSP2035 - Shipment \#3 Load, Decon, and Leak Test Overpacks

$\begin{array}{ll}\text { AS } & \text { ASSIST } \\ \text { ENT } & \text { DNTRY PERSON } \\ \text { RPY } & \text { RET } \\ \text { OND } & \text { UNDRESSER }\end{array}$

TOTAL BCSP2035

BCSP2040 - Shipment \#3 Prep, Load, and Leak Test Cask

$\begin{array}{ll}\text { AS } & \text { ASSIST } \\ \text { ENT } & \text { ENTIRY DERSON } \\ \text { RFT } & \text { RCT } \\ \text { OND } & \text { UNDRESSER }\end{array}$

TOTAL BCSP2040

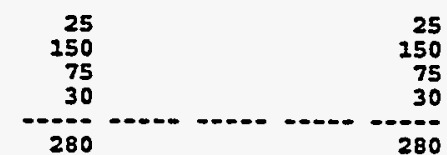

BCSP2050 - Shipwent \#4 Load, Decon, and Leak Test Overpacks

$\begin{array}{ll}\text { AS } & \text { ASSIST } \\ \text { ENT } & \text { ENTRY PERSON } \\ \text { RPT } & \text { RCT } \\ \text { UND } & \text { UNDRESSER }\end{array}$

TOTAL BCSP2050
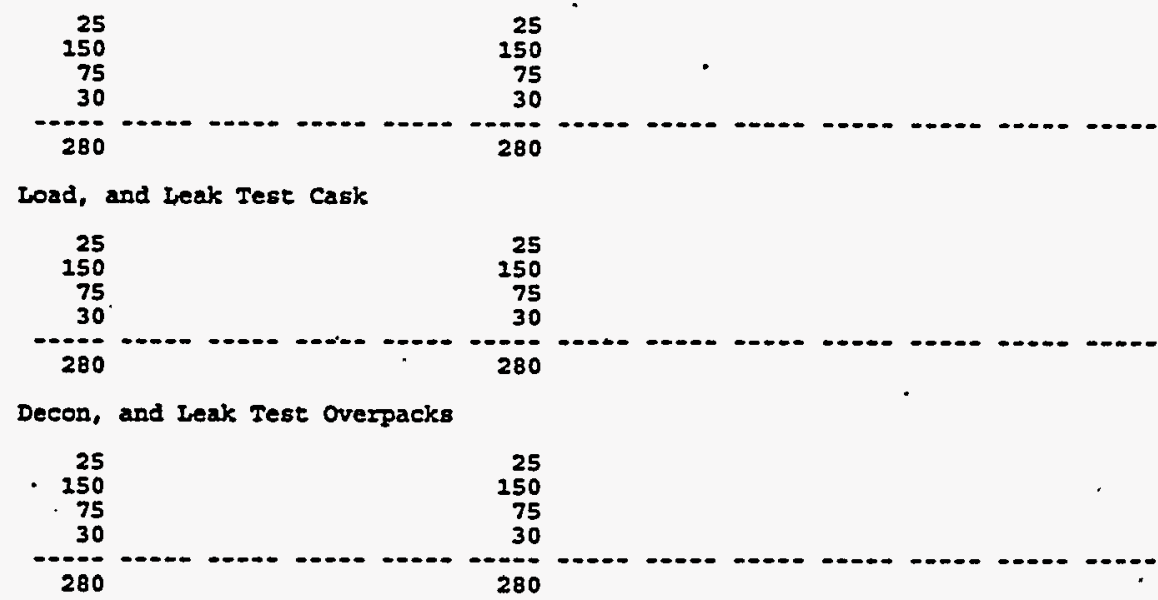


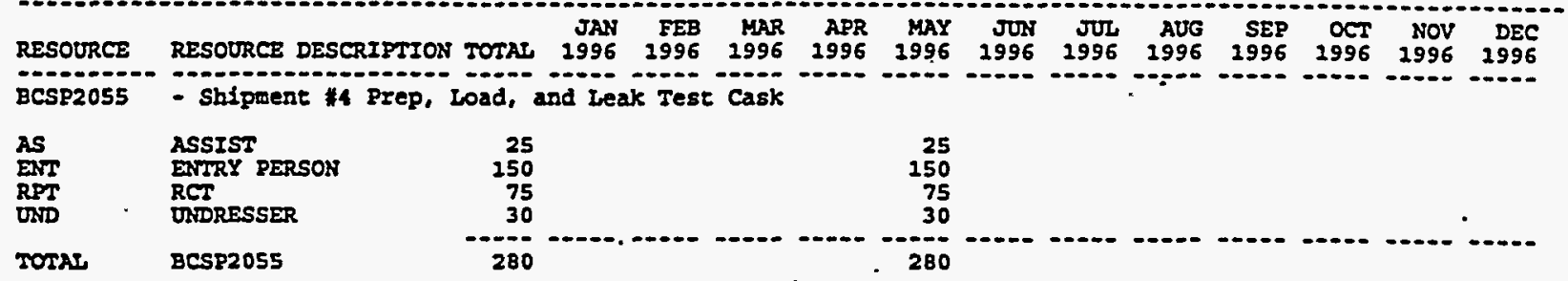

BCSP2065 - Shipwent \$5 Load, Decon, and Leak Test Overpacks

$\begin{array}{ll}\text { AS } & \text { ASSIST } \\ \text { ENT } & \text { ENIRY PERSON } \\ \text { RPI } & \text { RCT } \\ \text { UND } & \text { UNDRESSER }\end{array}$

TOTAL BCSP2065

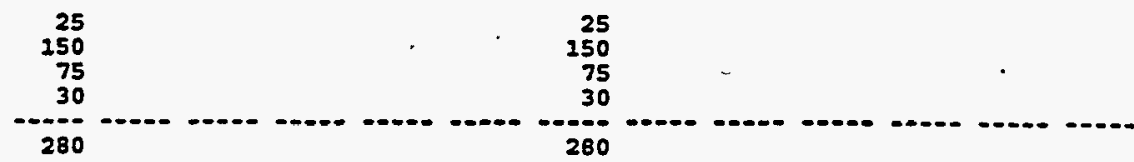

BCSP2070 - Shipaent 5 Prep, Load, and Leak Test Cask

$\begin{array}{ll}\text { AS } & \text { ASSIST } \\ \text { ENT } & \text { ENTRY PERSON } \\ \text { RPT } & \text { RCT }\end{array}$

$\begin{array}{ll}\text { RPT } & \text { RCL } \\ \text { UND } & \text { UTDRESSER }\end{array}$

TOTAZ BCSP2070

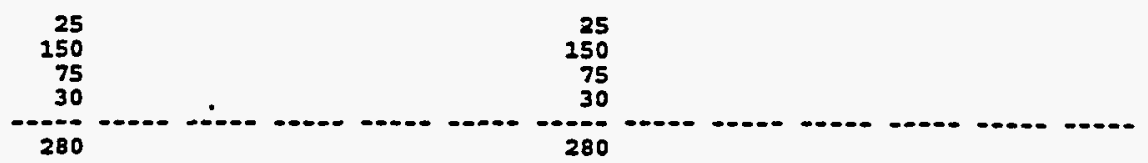

BCSP2080 - Shipment \$6 Load, Decon, and Ieak Test Overpacks

AS ASSIST

$\begin{array}{ll}\text { ENT } & \text { ASSIST } \\ \text { RPT } & \text { RCT }\end{array}$

UTD UTDRESSER

TOTAL BCSP2080

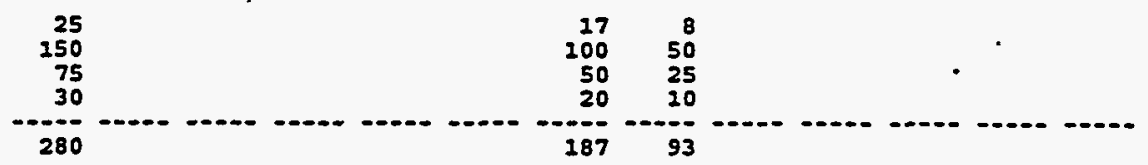

BCSp2085 - Shipment $\% 6$ prep, Load, and Leak Test Cask

AS ASSIST

ENT ENTRY PERSON

RPT RCT

OND TNDRESSER

TOTAL . BCSP2085

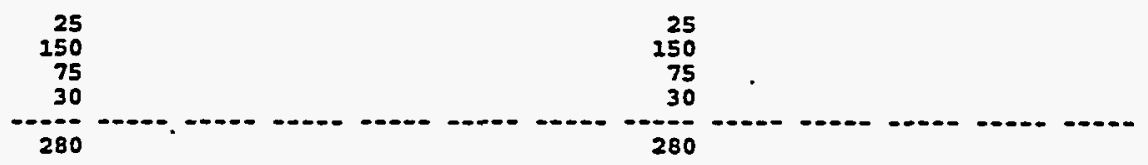

BCSP2095 - Shipment \#7 Load, Decon, and Leak Test Overpacks

AS ASSIST

ENT

$\begin{array}{ll}\text { RPT } & \text { RCT } \\ \text { UND } & \text { UNDRESSER }\end{array}$

TOTAL BCSP2095

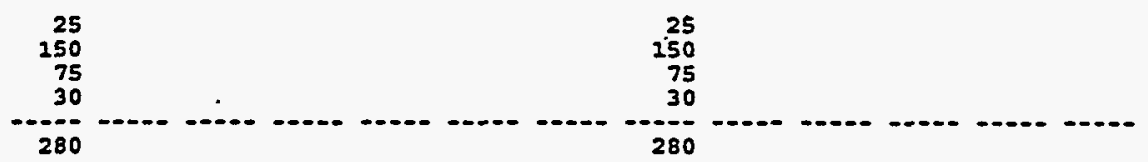

BCSP2100 - Shtpment \#7 Prep, Load, and Leak Test Casik

$\begin{array}{ll}\text { AS } & \text { ASSIST } \\ \text { ENT } & \text { ENTRY PERSON } \\ \text { RPT } & \text { RCT }\end{array}$

$\begin{array}{ll}\text { RPT } & \text { RCT } \\ \text { UND } & \text { UNDRESSER }\end{array}$

TOTAL BCSP2100

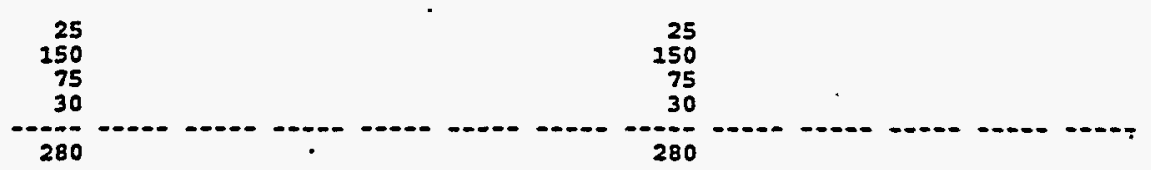

DC400425 - D-Cell Crane Prev. Maint.

AS ASSIST

EI ELECTRICIAN

TOTAL DC400415

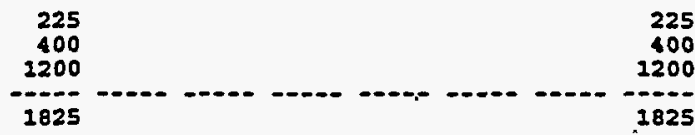


RESOURCB LOADING DETAIL BY ACTIVITY - PNL $\begin{array}{llllllllll} & & & \end{array}$ EC400415D6 - E-Cell Par Prev. Maint.

EI ELECTRICIAN

MH MILIWRIGET

RPI RCF

TECH ROT CETL TECHIICIAN

TOTAT EC400415D6
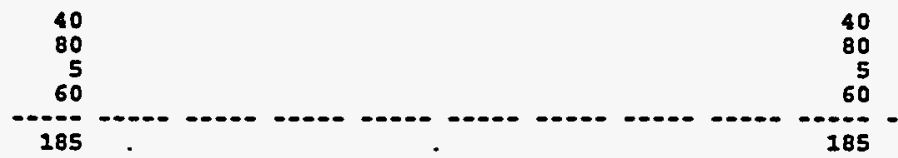

III

20 - Install in Cell Equip. gallery services

AS ASSIST

ENT ENIRY PERSON

RFT

UND UNDRESSER

TOTAL 20

$\begin{array}{r}20 \\ 200 \\ 75 \\ 30 \\ \hline 225\end{array}$

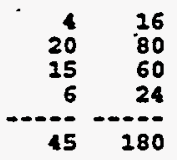

HI

33 - Install vacuum Tank in D-Cell

As ASSIST

ENT ESTISY PERSON

$\begin{array}{ll}\text { RPT } & \text { RCF } \\ \text { UND } & \text { UNDRESSER }\end{array}$

TOTAI 33

\begin{tabular}{rr}
20 & 20 \\
100 & 100 \\
75 & 75 \\
30 & 30 \\
\hline 225 & 225
\end{tabular}

Py260 - A-Erames

$A B$

RPT

TECH

TOTAT

AIR BaLANCE ROT CEI IECENICIAN

PN262 Ex260

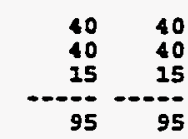

AB

RPT

TRCA

TOTAT

- A-Frames

P1264

A日

RPT

TECH

TOTAT

AIR BATANCE

RCT

HOT CEII IECENICIAN
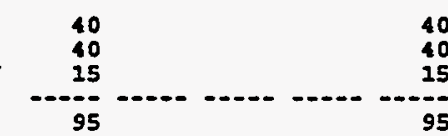

PN262

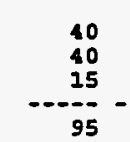

95

PN268 - Annual HEPA filcer DM

RPT

TECA

TOTAI

- A-Frames

PI296 * - REC DOOI PH

AS ASSIST

MH MILLWRIGET

RPT RCT

UND UNDRESSER

TOTAI PM296
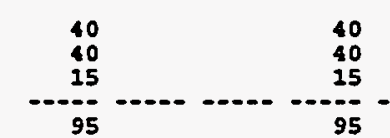

PH310 - B-Cell A-Frawe change

AB

AIR RALANCE
ASSIST

200
20

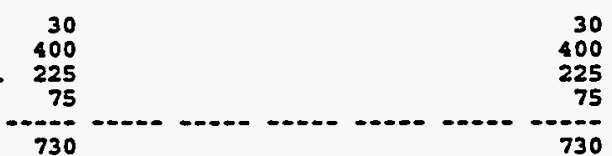

730

730

200

20 
RESOURCB RESOURCE DESCRTPTTON TOTA, JAN FEB MAR APR MAY JUN JUL AUG SEP OCT NOV DEC

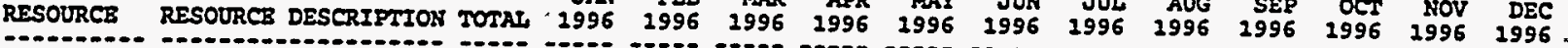
PH310 - B-CelI A-Prame change

Mit

RPT MILLWRIGHT

T2C: HOT CELl TECHNTCIAT

Torat PMI3

\begin{tabular}{rr}
100 & 400 \\
100 & 100 \\
300 & 300 \\
\hline 1020 & -1020
\end{tabular}

P19322 - D-Cell X-Exame cable repair

AB AIR BAtHANCE

AS ASSIST

MH MILWWIGKI

RPT RCT

IECA

Torat ROT

$\begin{array}{r}200 \\ 20 \\ 100 \\ 200 \\ 300 \\ \hline 1020\end{array}$

$\begin{array}{r}200 \\ 20 \\ 400 \\ 100 \\ 300 \\ \hline 1020\end{array}$

PM330

AB

As

MH

RPT EM322 100

HOT CELI TECHNICIAN

$\begin{array}{lr}\text { - B-CelI A-Frame change } \\ \text { AIR BALANCB } & 200 \\ \text { ASSIST } & 20 \\ \text { MILILRIGET } & 400 \\ \text { RCT. } & 100 \\ \text { HOT CEL TECHNICIAN } & 300 \\ & -1000 \\ \text { PH330 } & 1020\end{array}$

PN 18 - Ship Consolidaced SERF-FFIF Pins from 327 to 324

RPT

TECH

TOTAT RCT
HOT CELT TECHICIAN PN 18
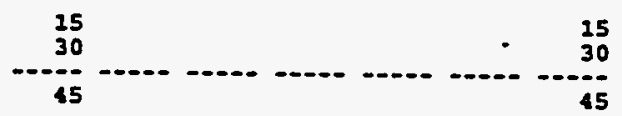

IN

20 - Receive 29 Fuel Pins from ANL-W 324

$\begin{array}{ll}\text { RPT } & \text { RCT } \\ \text { IECH } & \text { HOT CELT TECHNICIAN }\end{array}$

TosAt

E2T PN

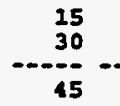

45

$\begin{array}{ll}\text { RPT } & \text { RCT } \\ \text { TECH } & \text { HOT CELI TECHNICIAN }\end{array}$

TOTAI PN 21

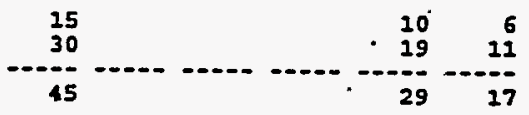

PN 23 - Store C-I Shipment at 324

RPT RCT 15

TECH HOT CELI TECHICIAN 3

TOTAL PN $23 \quad 45$

FN 24 - Ship C-1 Shipment to FETE

RPT RCT 15

$\begin{array}{lll}\text { TECH } & \text { HOT CELL TECHIICIAN } \\ \text { TOTAL } & \text { PN } & 24\end{array}$

F2 25 - Ship MFA-1/MEA-2/ACO-3 Fuel Pins to FFTE

$\begin{array}{ll}\text { RPT } & \text { RCT } \\ \text { TECI } & \text { HOT CELI TECTNICIAN }\end{array}$

TOTAL

FN 25

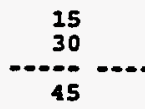

\begin{tabular}{rrrr}
5 & 4 & 5 & 1 \\
10 & 9 & 9 & 3 \\
\hline 14 & 13 & 14 & 4
\end{tabular}

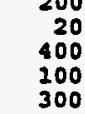

300

1020

22

25

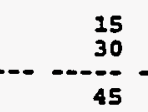

45
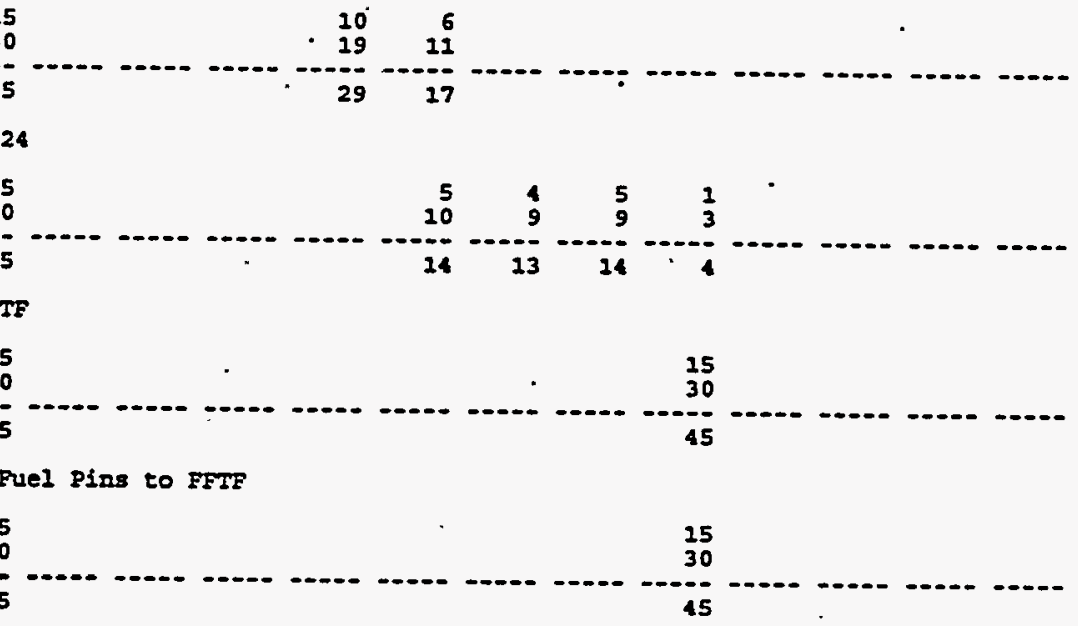


JAN FEB MAR APR MAY JUN JUL AUG SEP OCT NOV DEC

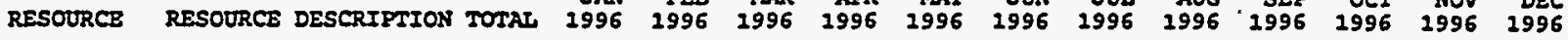
FN 26 - Ship PNC Wagte (SERF CEll Fuel Pins) to FFTF

$\begin{array}{lr}\text { RPT } & 15 \\ \text { TECH } & 30 \\ \text { TOT CELS TECHICIAN }\end{array}$

TOTAI $26 \quad 45$

FA 27 - Receive MFA-2 DS at 324 bldg from ANL-W

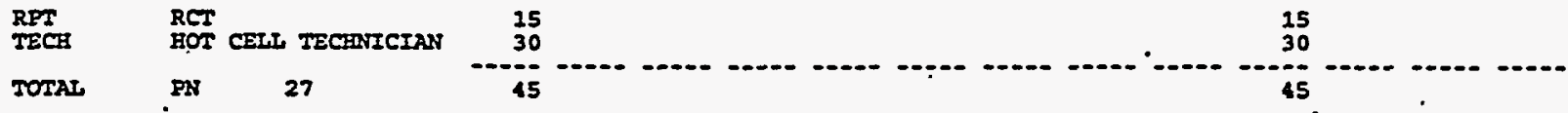

FN 124 - Xfer Pkgd Fuel Pins to G-Cell or SMF for cing

$\begin{array}{llll}\text { RPT } & \text { RCT } & 15 & 15 \\ \text { TECH } & \text { BOT CELT TECERICIAN } & 30\end{array}$

TOTAL BN 114 .

DN 115 - Iransfer Packaged Fuel to SMF 1324

- RET RET ROT CEIT TECTRICIAN

TOTAY PN $115 \quad 45$

sc400415 - s-Cell Par Prev. Maint.

$\begin{array}{ll}\text { AS } & \text { ASSIST } \\ \text { EI } & \text { ELECIRICIAN } \\ \text { MT } & \text { MILLWRIGHT } \\ \text { RPT } & \text { RCT } \\ \text { TECH } & \text { HOT CEHI TECHNICIAN } \\ \text { UND } & \text { UNDRESSER } \\ \text { TOTAL } & \text { SC400415 }\end{array}$

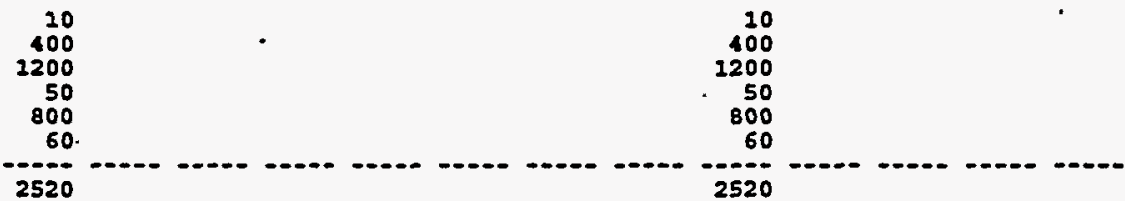

SC400416 - S-Cell Crane Prev. Maint.

AS ASSIST

EL ELECTRICIAN

MW MILLWRIGIT

RPI RCI

TECH HOT CEIT TECENICIAN

UND TIDRESSER

TOTAL $\quad \mathrm{SC400416}$

Maint
10
400
1200
50
800
60
2520

15
30
-05

SF108 - Waste Disposal

$\begin{array}{ll}\text { RPT } & \text { RCT } \\ \text { TECA } & \text { HOT CELL TECFIICIAN }\end{array}$

TOTAL SFI08

SF109 - Waste Disposal

RPT

- TECH

IOTAT

RCT ROT TEW TECINICIAN
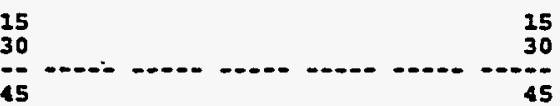

15
30
45

SF110 - Haste Disposal

RPT

TOTAL

RCT

SE120

10
20
-0
30 $\quad \begin{array}{r}10 \\ 20\end{array}$




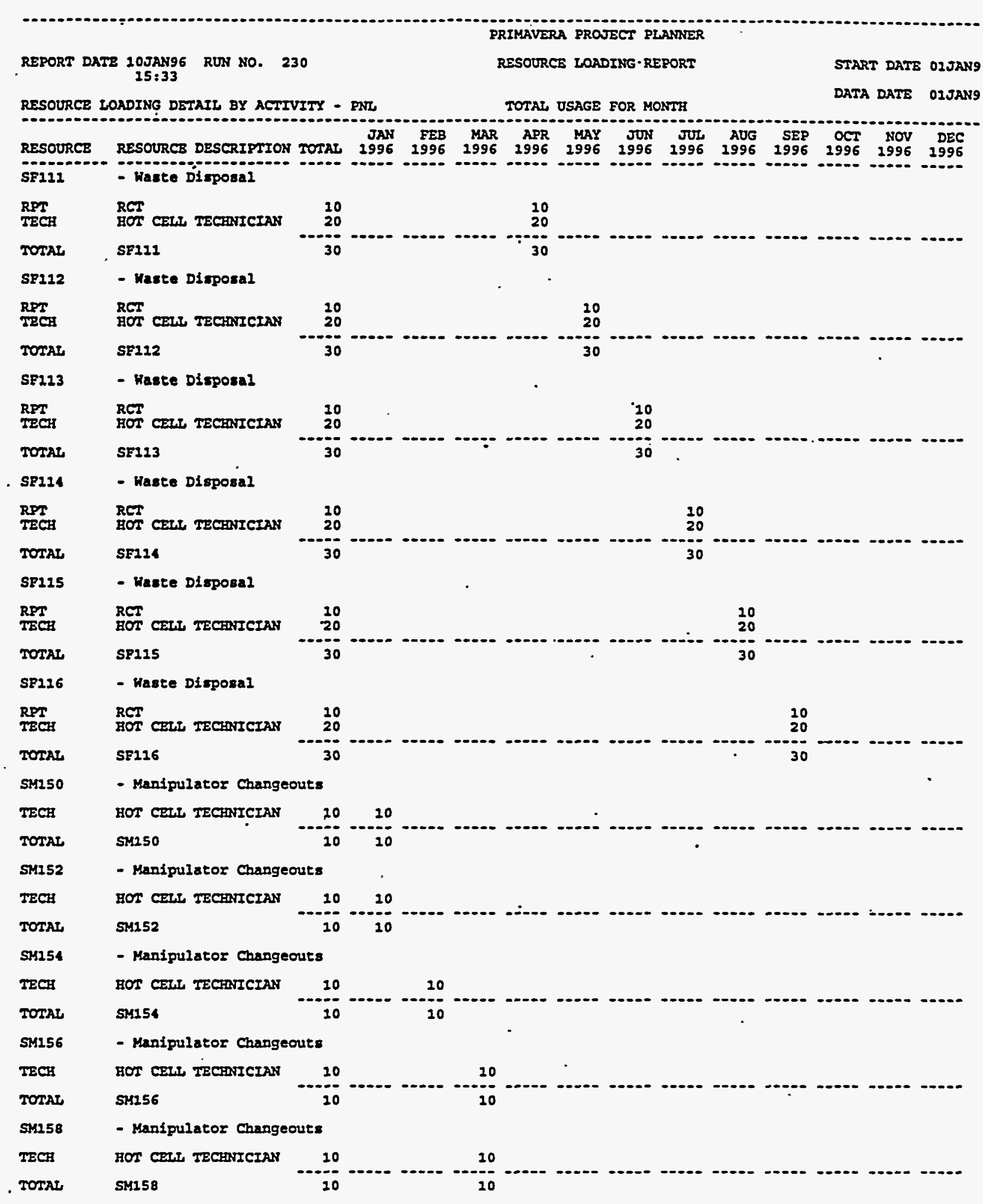




\begin{tabular}{|c|c|c|c|c|c|c|c|c|c|c|c|c|c|c|}
\hline RESOURCB & RESOURCE DESCRIPTION & TOTAL & $\begin{array}{r}\text { JAN } \\
1996\end{array}$ & $\begin{array}{r}\text { FEB } \\
1996\end{array}$ & $\begin{array}{r}\text { MAR } \\
1996\end{array}$ & $\begin{array}{c}\text { APR } \\
1996\end{array}$ & $\begin{array}{r}\text { MAY } \\
1996\end{array}$ & $\begin{array}{l}\text { JN } \\
1996\end{array}$ & $\begin{array}{r}\pi J \\
1996\end{array}$ & $\begin{array}{l}\text { AUG } \\
1996\end{array}$ & $\begin{array}{r}\text { SEP } \\
1996\end{array}$ & $\begin{array}{l}\text { OCT } \\
1996\end{array}$ & $\begin{array}{l}\text { Nov } \\
1996\end{array}$ & $\begin{array}{r}\text { DEC } \\
1996\end{array}$ \\
\hline $\sin 160$ & - Manipulator Changec & uts & & & & 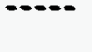 & . & & 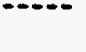 & 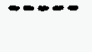 & 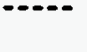 & & & \\
\hline TECH & HOT CELL TECENICIAN & 20 & & & & 10 & & & & & & & & \\
\hline TOTAL & SM160 & 10 & & & & 10 & & & & & & & & \\
\hline SM162 & - Manipulator Changed & uts & & & & & & & & & & & & \\
\hline TECH & HOT CELI TECENICIAN & 20 & & & . & & 10 & & & & & & & \\
\hline TOTAL. & SH162 & 10 & & & & & 10 & & & & & & & \\
\hline $\operatorname{SN} 164$ & - Manipulator Changed & iuts & & & & & & & & & & & . & \\
\hline TECH & HOT CELI TECERICIAN & 10 & & & & & 10 & & & & & & & \\
\hline TOTAL & $\sin 64$ & 10 & & & & & 10 & & & & & & & \\
\hline SM166 & - Manipulator Changeo & uts: & & & & & & & & & & & & \\
\hline TECH & HOT CELI TECENICIAN & 10 & & & & & & 10 & & & & & & \\
\hline TOTAI & $\sin 66$ & 10 & & & & & & 10 & & & & & & \\
\hline $\operatorname{Sin} 68$ & - Manipulator Changec & uts & & & & & & & & & & $\cdot$ & & \\
\hline TECH & EOT CELI TECENICIAN & 10 & $\cdot$ & & & & & & 10 & & & & & \\
\hline TOTAI & $\operatorname{SM168}$ & 30 & & & & & & & 20 & & & & & \\
\hline SN270. & - Manipulator Changec & uts & & · & & & & & & & & & & \\
\hline TECH & HOT CELL TECHNICIAN & 10 & & & & & & & 10 & & & & & \\
\hline TOTAL & SHI 70 & 10 & & & & & & & 10 & & & & & \\
\hline $\sin 272$ & - Manipulator Changec & ues & & & & & & & & & & & & \\
\hline IECH & EOT CELT TECFNICIAN & 10 & & & & & & & 20 & & & & & \\
\hline TOTAL & SMI 72 & 10 & & & & & & & 10 & & & & & \\
\hline SN274 & - Manipulator Changed & uts & & & & & & & & & $\cdot$ & & & \\
\hline TECH & BOT CELL TECHNICIAN & 10 & & & & & & & & 10 & & & & \\
\hline TOTAL & $\sin 74$ & 10 & & & & & & & . & 10 & & & & \\
\hline $\sin 176$ & - Manipulator Changeo & uts & & & & & & & & & & & & \\
\hline IECH & HOT CELI TECENICIAN & 10 & & & & & & & & & 10 & & & " \\
\hline TOTAL & $\operatorname{SM176}$ & 10 & & & & & & & & & 10 & & & \\
\hline 5,5200 & - Manipulator Changec & ute & & & & & & & & & & & & \\
\hline TECH & HOT CELI TECENICIAN & 10 & & & & & & & & & & 20 & & \\
\hline TOTAL & $\operatorname{SN200}$ & 10 & & & & & & & . & & & 20 & & \\
\hline $\sin 244$ & - Manipulator Changed & uts & & & & & & & & & & & & \\
\hline TECH & HOT CELL TECENICIAN & 10 & & & & & & & & & & & 10 & \\
\hline TOTAI & SM244 & 10 & & & & & & & & & & & 10 & \\
\hline $5 \times 246$ & - Manipulator Changec & uts & & & & & & & & & & & & \\
\hline TECH & HOT CELS TECHNICIAN & 10 & & & & & & & & & •" & & & 10 \\
\hline TOTAZ & SL:246 & 10 & & & & & & & & & & & & \\
\hline
\end{tabular}




\begin{tabular}{|c|c|c|c|c|c|c|c|c|c|c|c|c|c|c|}
\hline RESOURCE & RESOURCE DESCRIPTION & TOTAY & $\begin{array}{r}\text { JAN } \\
1996\end{array}$ & $\begin{array}{r}\text { FEB } \\
1996\end{array}$ & $\begin{array}{r}\text { MAR } \\
1996\end{array}$ & $\begin{array}{r}\text { APR } \\
1996\end{array}$ & $\begin{array}{r}\text { MAX } \\
1996\end{array}$ & $\begin{array}{r}\text { JUN } \\
1996\end{array}$ & $\begin{array}{r}\pi \pi \\
1996\end{array}$ & $\begin{array}{r}\text { AUG } \\
1996\end{array}$ & $\begin{array}{r}\text { SEP } \\
1996\end{array}$ & $\begin{array}{r}\text { OCT } \\
1996\end{array}$ & $\begin{array}{l}\text { NOV } \\
1996\end{array}$ & $\begin{array}{r}\text { DEC } \\
1996\end{array}$ \\
\hline $5 \times 342$ & - Misc. Cask Load/Un] & load & & & & & & & & $\because-\infty$ & $-\infty+\infty$ & 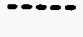 & $-\infty+\infty$ & \\
\hline IECA & HOT CELI TECINICIAN & 25 & & & 25 & & & & & & & & & \\
\hline TOTAL & SM342 & 30 & & & 30 & & & & & & & & & \\
\hline
\end{tabular}

Sw344 - Mise. Cask Load/Unload

$\begin{array}{ll}\text { RPT } & \text { RCT } \\ \text { TECH } & \text { HOT CELI TECENICIAN }\end{array}$

TOTAI SH344 30

Sx346 - Misc. Cask Load/Unload

RPT

TEC

TOTat

RCT

5
25
-0.5

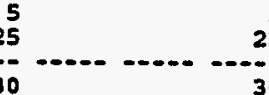

Sm348 - Misc. Cask Ioad/Unload

RPT RCT .

TECH HOT CEL TECHNICIAN

TOTAT $5 \% 348$

5
25
-50
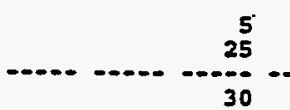

SM350 - Misc. Cask Ioad/Onload

RPI

IECA

TOTAL

$$
\text { RCT }
$$

RCT
HOT CELT TECHNICIAN

$\begin{array}{r}5 \\ 25 \\ -0-0 \\ \hline\end{array}$

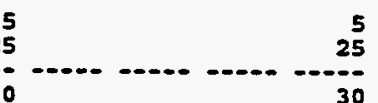

SM352 - Misc. Cask Load/Onload

RFT RCF 5

TOTAI SM352

SM354 - Misc. Cask Load/Onload

RPT. RCT

TECH HOT CELL TECENICIAN 25

TOTAL SM354

SM356 - Hisc. Cask Load/Unioad

$\begin{array}{ll}\text { RPT } & \text { RCT } \\ \text { IECE } & \text { HOT CELL TECHNICIAN } 25\end{array}$

TOTAL SM356 30

5

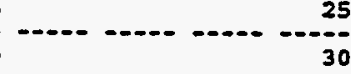

SK358 - Misc. Cask Load/Onload

RPT $\quad$ RCT

TECH BOT CELI TECHNICIAN

SM360 - Mise. Cask Load/Onload

RPT RCT :

TECH HOT CELI TECHICIAN

TOTAT SM360 30

SM362 - Misc. Cask Load/Onload

RPT RCT
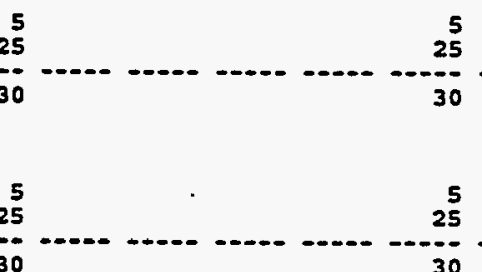

30

RT 


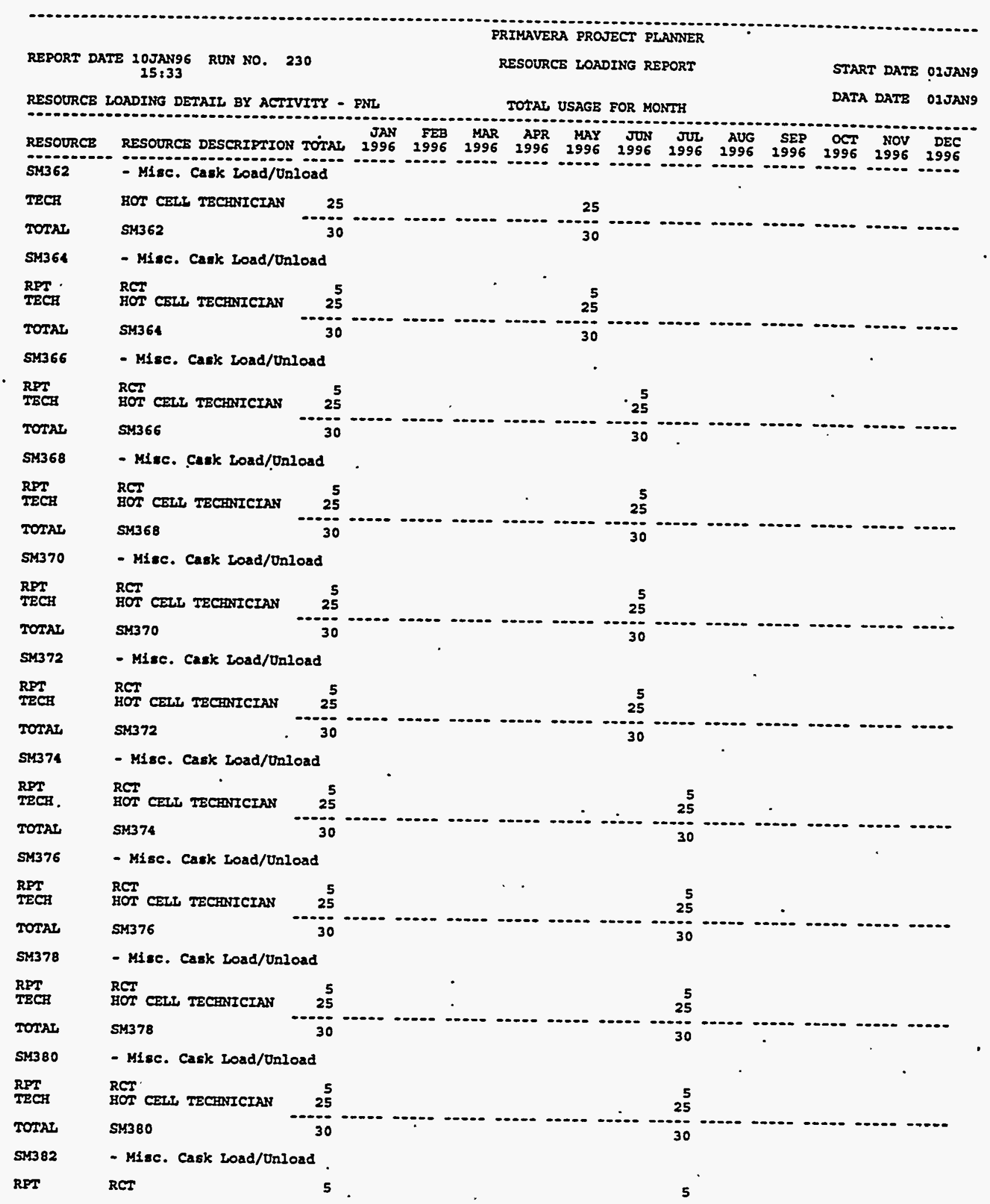




\title{
RESOURCE
}

\section{LOADING}

\author{
DETAIL
}

\section{BY RESOURCE}

\section{PNNL}


DETAIL BY RESOURCE

\section{TOTAL USAGB FOR MONTH}

\begin{tabular}{|c|c|}
\hline $\begin{array}{l}\text { ACP ID } \\
\text { CRAFT }\end{array}$ & $\begin{array}{l}\text { DESC } \\
- \text { CRAFT }\end{array}$ \\
\hline $\mathbf{A B}$ & - AIR BALARICB \\
\hline 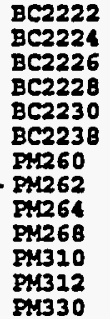 & $\begin{array}{l}\text { HEPA A-Frame Dos } \\
\text { HEPA A-Frame DOs } \\
\text { HEPA A-Frame DOs } \\
\text { HEPA A-Erame DOs } \\
\text { HEPA A-Frame DOs } \\
\text { HEPA A-Frame DOs } \\
\text { A-Frames } \\
\text { A-Frames } \\
\text { A-Frames } \\
\text { Angual HEPA E1lter } \\
\text { B-Cell A-Frame chang } \\
\text { D-Cell A-Frame cable } \\
\text { B-Cell A-Frame chang }\end{array}$ \\
\hline
\end{tabular}

TOTAT.

JA9

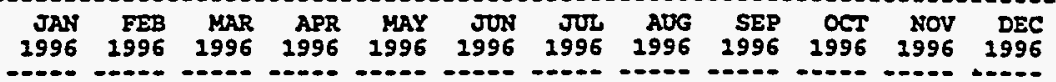

\section{subTorat, $A B$}

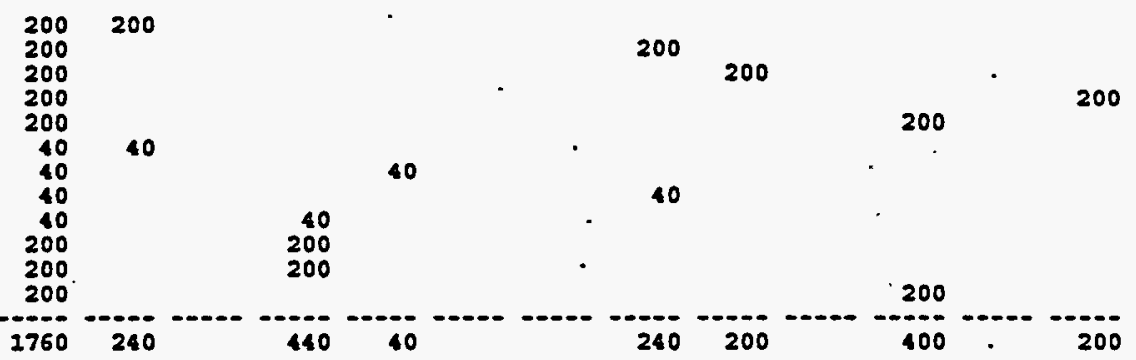

\section{EL - ELECTRICIAN}

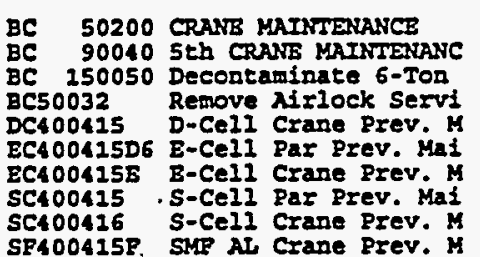

-

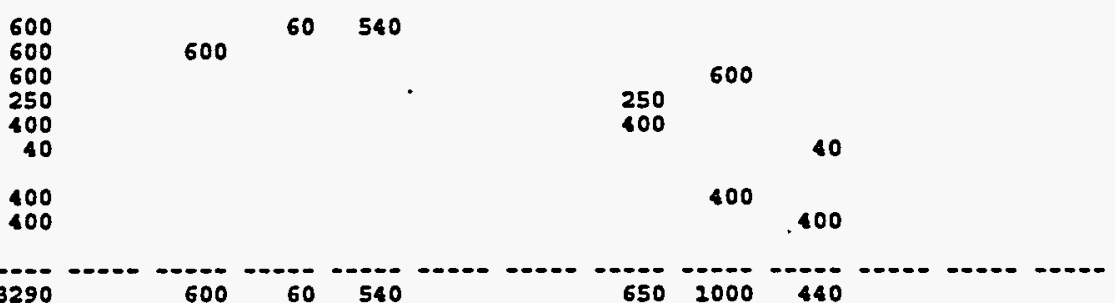

SUBTOTAL EL

3290

$600 \quad 60 \quad 540$

$6502000 \quad 440$

\section{Mi - MITLARIGET}

BC 28191 RM11POG Filter Chang

BC 28193 RMI1POG Filter Chang

BC 28194 RMIIPOG Filter Chang

BC 28195 RMIIPOG Filter Chang

BC 50200 CRANB MATNIENANCE

BC 90040 5th CRANE MAINTENANC

150050 Decontaminate 6-Ton

600220 Remove Pipetrench Co

BC 600230 SPRAY DOWN COVER BLO

BC120 Maipulator Decon an

BC122 Manipulator Decon an

BC125 Manipulator Decon an

BC126 Manipulator Decon an Kanipulator Decon an Manipulacor Decon an Hanipulator Decon an Manipulator Decon an Manipulator Decon an Manipulator Decon an Manipulator Decon an Manipulator Decon an Manipulator Decon an Manipulacor Changeou Manipulator Changeou Manipulator Changeou Manipulator Changeou Manipulator Changeou Manipulator Changeou Manipulator Decon an Manipulator Changeou Manipulator Decon an Manipulator Changeou Manipulator Decon an Manipulator Changeou Mantpulator Changeou Mantpularor Changeou Manipulator Changeou

80
80
80
2200
2200
2200
250
45
45
45
45
45
45
45
45
45
4

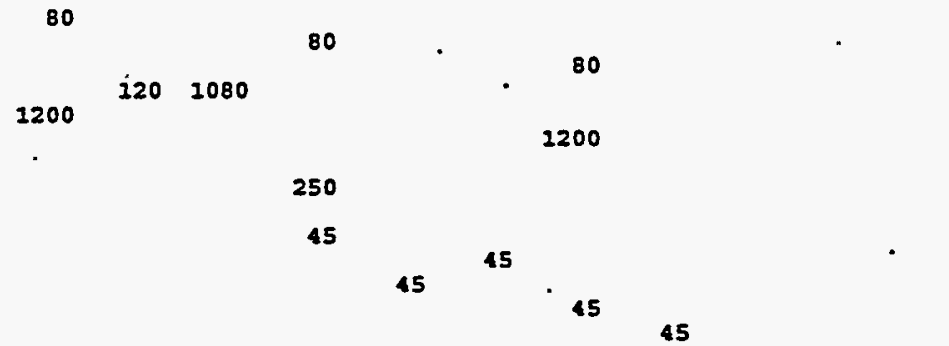

45

45

45

45

45

10

10

10

10 
PRIMAVERA PROJECT PLANIER

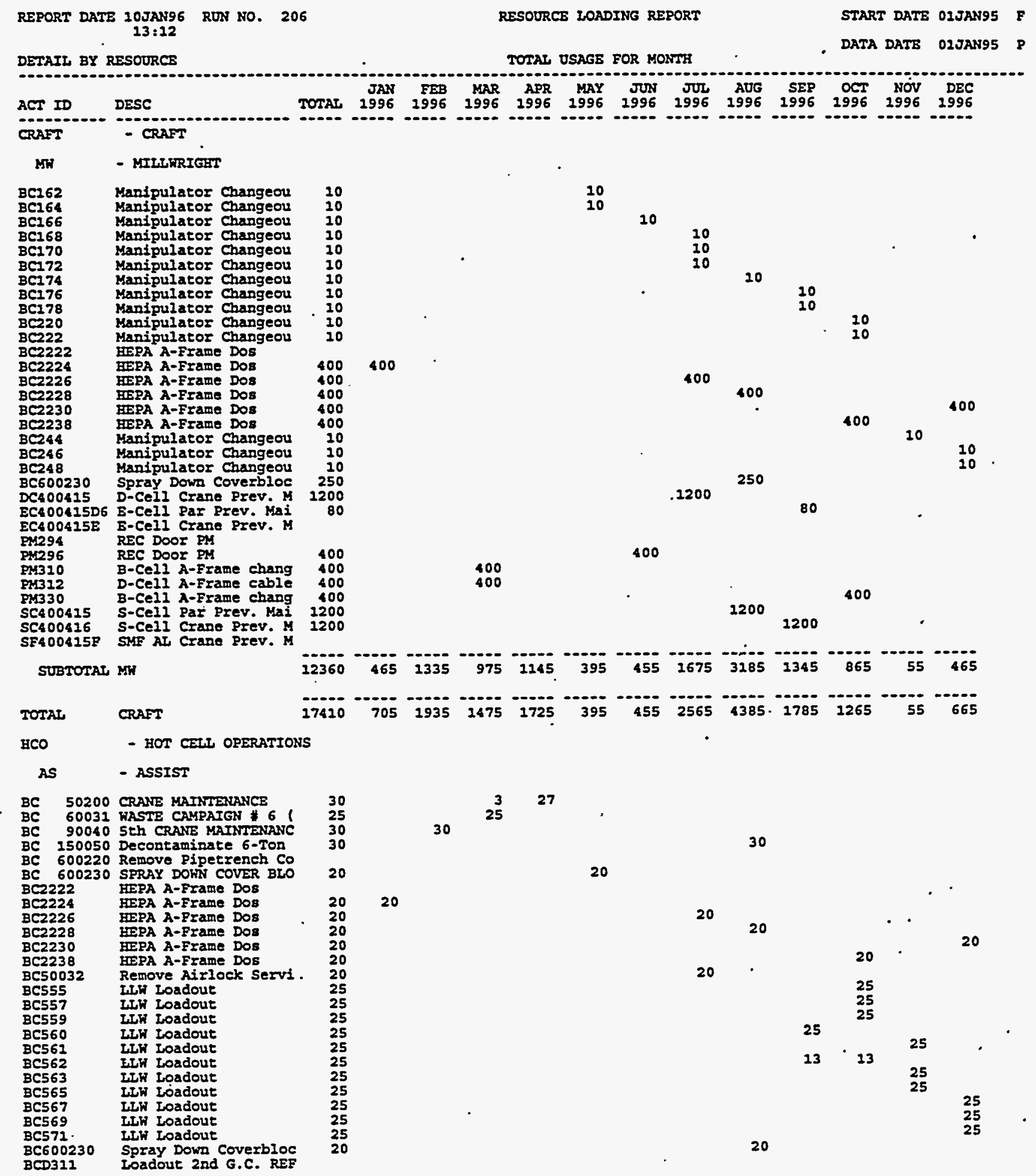




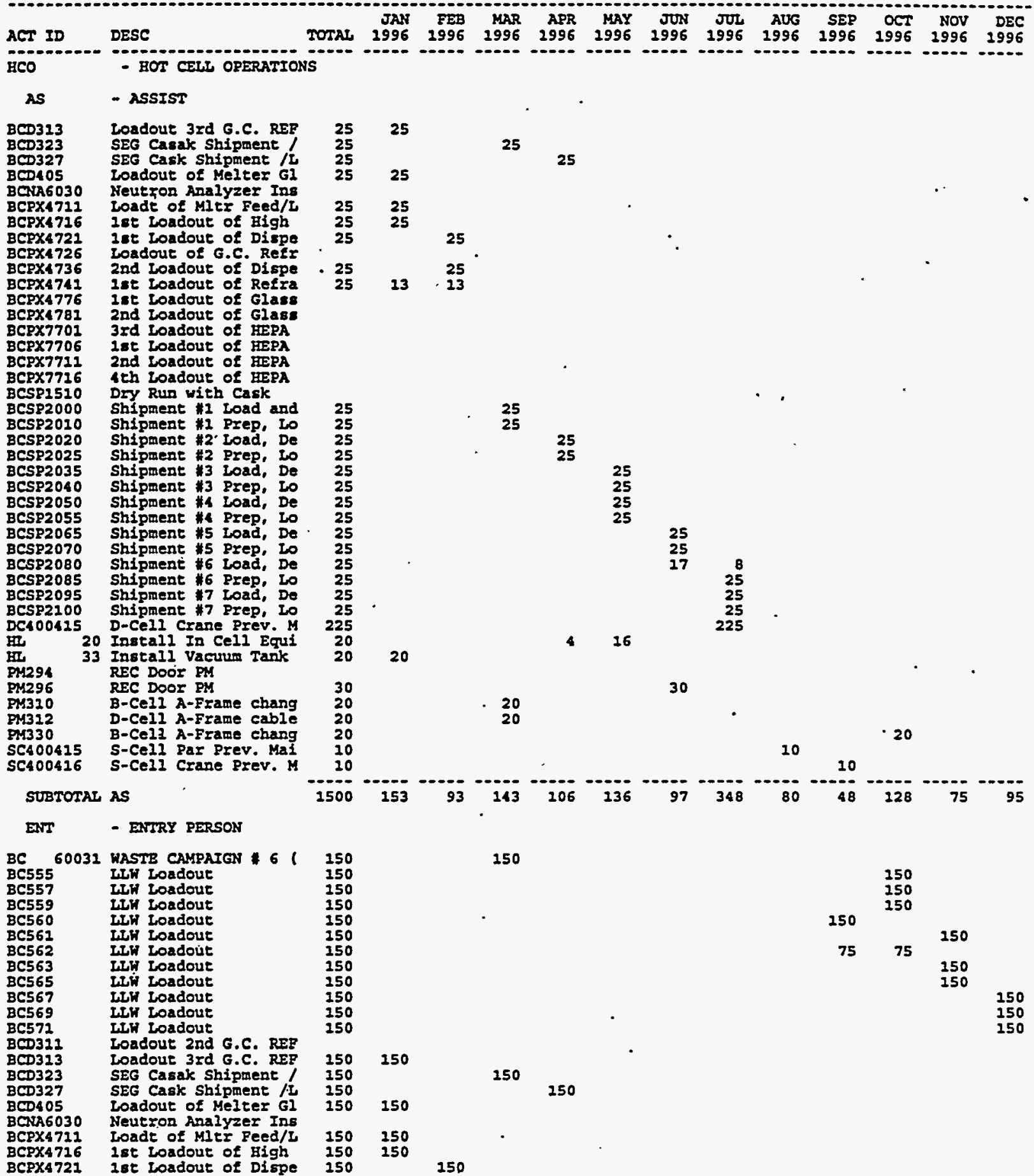


PRIMAVERA PROJECT PLANNER

REPORT DATE IOJAN96 RUN NO. 206

RESOURCE LOADING REPORT

START DATE 0IJAN9S F

$13: 22$

TOTAI USAGE FOR MONTH

DATA DATE O2JAN95 P

DETAIL BY RESOURCE

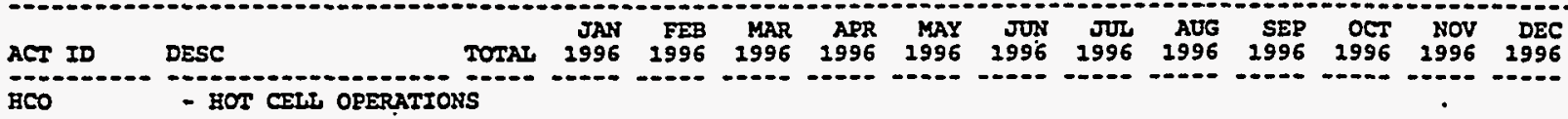

ENT - ENTRY PERSON

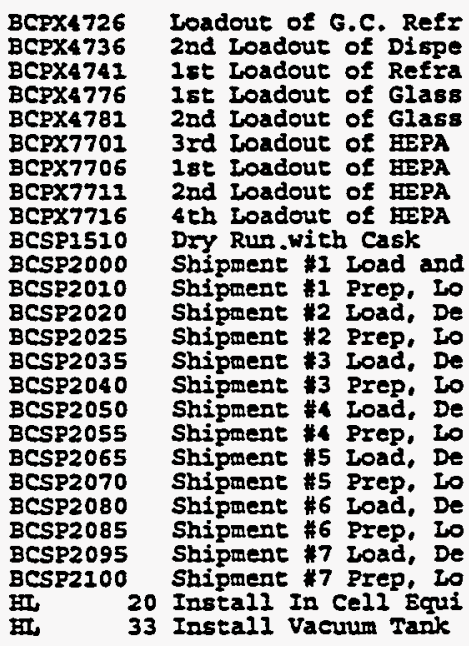

SUBTOTAT ENT

$\begin{array}{rrr}150 & & 150 \\ 150 & 75 & 75\end{array}$

IA

$\mathrm{BC} 120$
$\mathrm{BC} 122$

BC225

BC126

BC127

BC128

BC229

$8 \cos 30$

BC131

$\mathrm{BC} 132$

$\mathrm{BC} 133$

BC134

BC149

BCI51

BC153

SUBTOTAL IN

TECH - HOT CELI TECHNICIAN

BC 28191 RM11POG Filter Chang

BC 28194 RMIIPOG Filter Chang

BC 28195 RM11POG Filter Chang

BC140 Manipularor Changeor

BC142 Manipulator Changeou

BC144

BC146

BC146

$\mathrm{BC} 148$

BC150

BC152

BC254

BC156

BC158

BCI60

BC162

BC164

Maripilator Changeou

Manipulator Chan Hanipulator Changeou Manipulator Changeou Manipulator Changeou Manipulator Changeou Manipulator Changeou Manipulator Changeou Manipulator Changeou Manipulator Changeou Manipulator Changeou

10
10
10


20
20
20
20
20
20
20
20

20

$\begin{array}{lll}150 & 150 & \\ 150 & 150 & \\ 150 & & 150 \\ 150 & & 150\end{array}$

150

150

150

150

350

150

150

150
150

150

100
100
100

$5450 \quad 775 \quad 375 \quad 600 \quad 470 \quad 680 \quad 400 \quad 500 \quad-350$

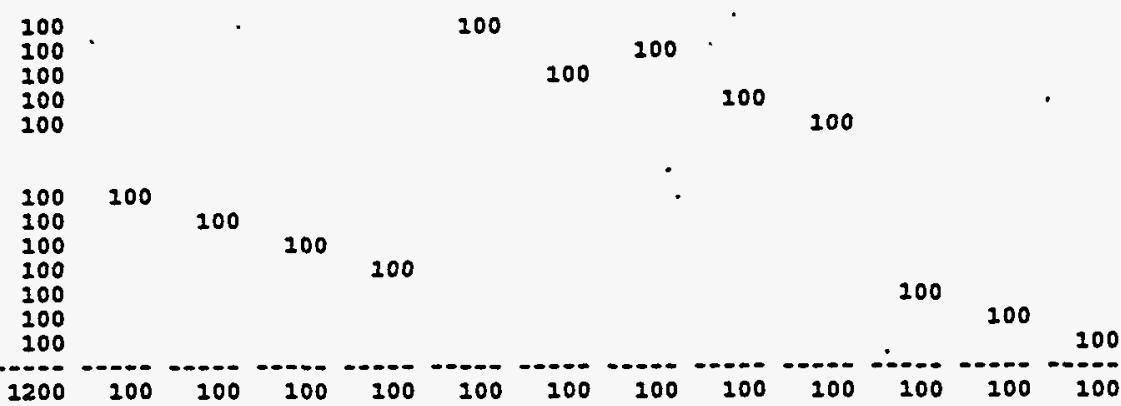

20

100

(.

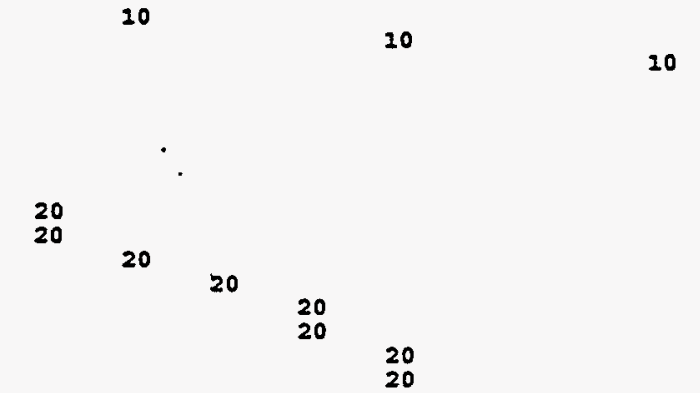




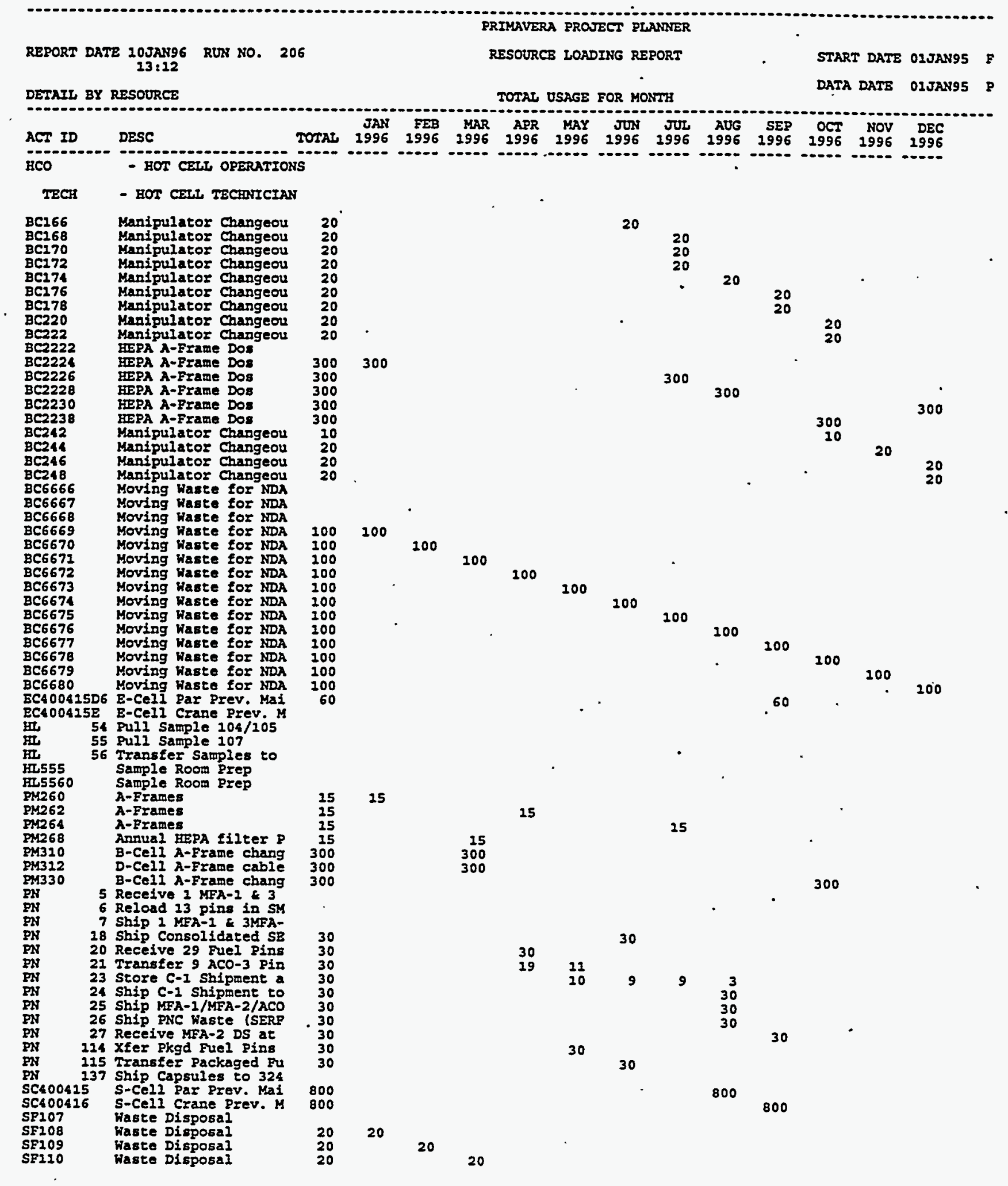

\section{B.41}


PRIMAVERA PROJECT PLANNER

REPORT DATE IOJAN96 RUN No. 206

RESOURCE LOADING RQEPORT

START DATE 0IJAN9S $P$

$13: 12$

TOTAL USAGE FOR MONTA

DATA DATE 02JAN95

DETAIL BY RESOURCE

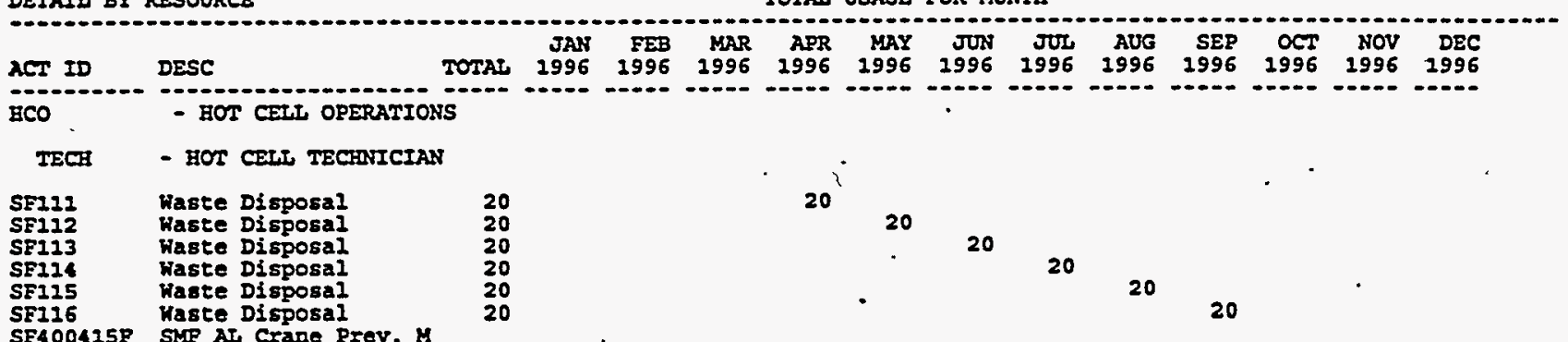

SF400415F SMF AI Crane Prev. $M$

SMI 0 Manipulator Changeou

SM142 Manipularor Changeor

SMI44 Manipulator Changeou

SN146 Manipulator Changeou

SM148 Manipulator Changeor

SM150 Manipulator Changeou

SM152 Manipulator Changeou

SMI54 Manipulator Changeou

SN156

Manipulator Changeou

Manipulator Changeou

Manipulator Changeou

Manipulator Changeou

Manipulator Changeor

Manipulator Changeou

Manipulator Changeou

Manipulator Changeou

Manipulator Changeou

Manipulator Changeou

Manipularor Changeou

Manipulator Changeou

Manipulator Changeou

Nanipulator Changeou

Manipulacor Changeou

Misc. Cask Load/unio

Misc. Cask Load/Unlo

Misc. Cask Load/Unlo

Misc. Cask Ioad/Onto

Misc. Cask Load/Unlo

Misc. Cask road/Onlo

Misc. Cask Ioad/Unlo

Hisc. Cask Ioad/Ualo

Misc. Cask Ioad/Unlo

Misc. Cask Load/Unlo

Misc. Cask Load/Onlo

Misc. Cask Load/Unlo

Misc. Cask Load/Unlo

Misc. Cask Load/Unlo

Misc.. Cask'Load/Unlo

Misc. Cask Load/Unlo

Misc. Cask Load/Unlo

Misc. Cask Load/Unlo

Misc. Cask Ioad/unio

Misc. Cask Load/Onlo

Misc. Cask Load/Unlo

Misc. Cask Load/Unlo

Misc. Cask Load/Unlo

Misc. Cask Load/Unlo

Misc. Cask Load/Unlo

Misc. Cask Load/Uni

Misc. Cask Load/Un

Mise. Cask Load/Unlo

Misc. Cask Load/Unio

Misc. Cask Load/Unlo

Misc. Cask Load/Unlo

Kisc. Cask Load/Unlo

Misc. Cask Load/Unlo

Misc. Cask Load/Ualo

Misc. Cask Load/Unlo

Misc. Cask Ioad/Unlo

Misc. Cask Load/Unlo

Misc. Cask Load/Unio

Misc. Cask Load/Unio

Misc. Cask Ioad/Unlo

10
10
10
10
10
10
10
10
10
10
10
10
10
10
10
10
10
10

10
10

10

10

10

10
10

10

10
10
10

10

10

20

10

20 


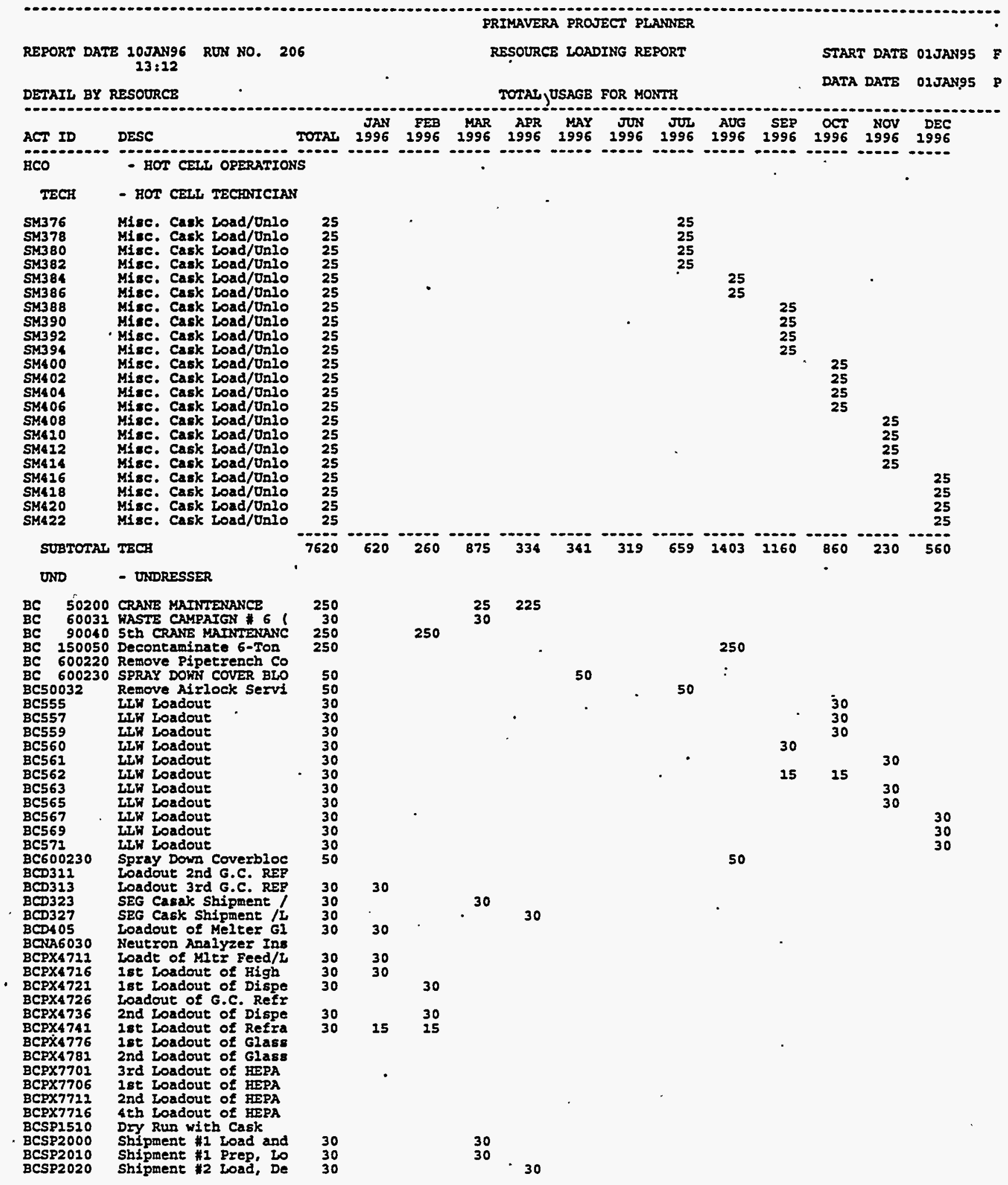




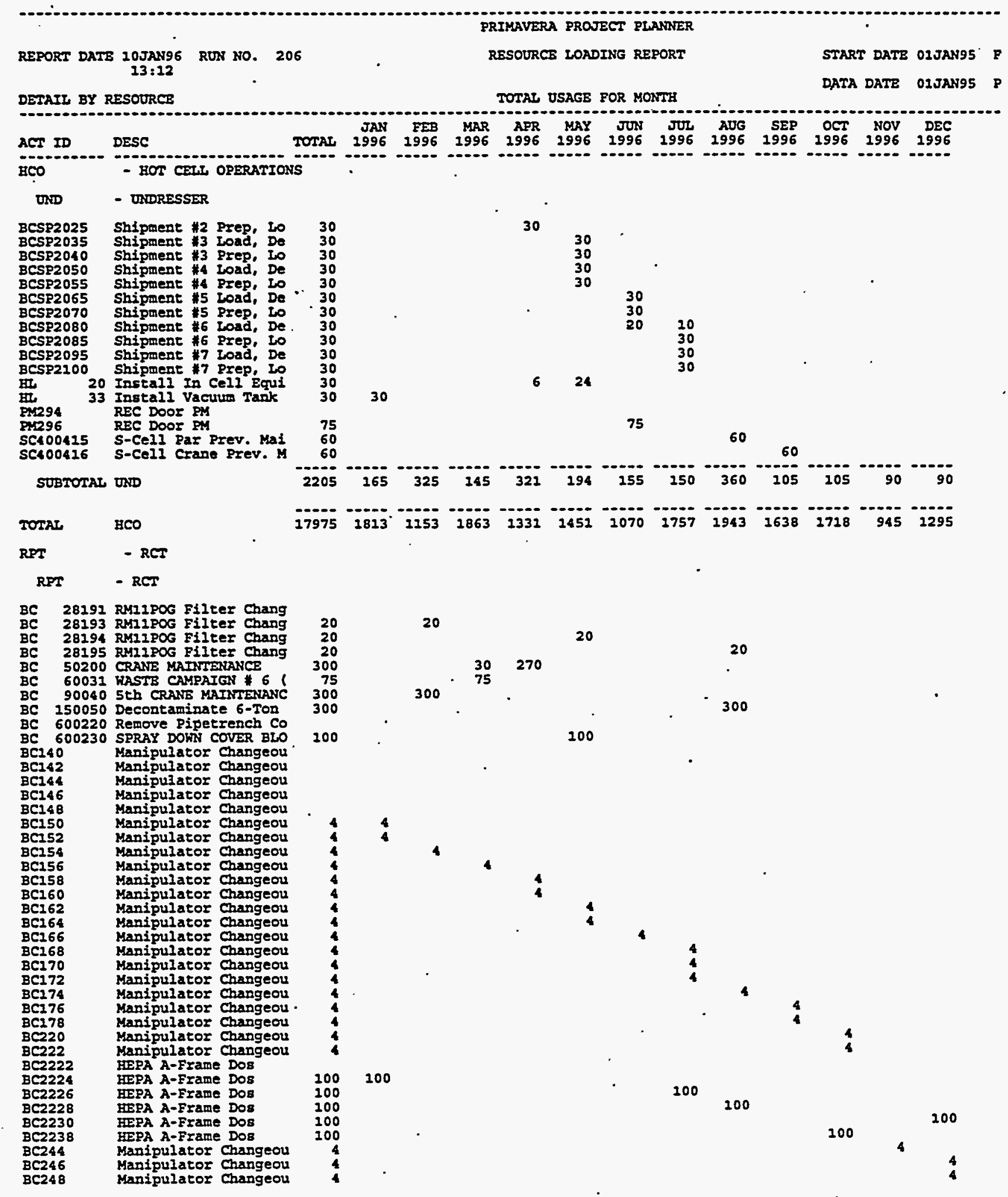




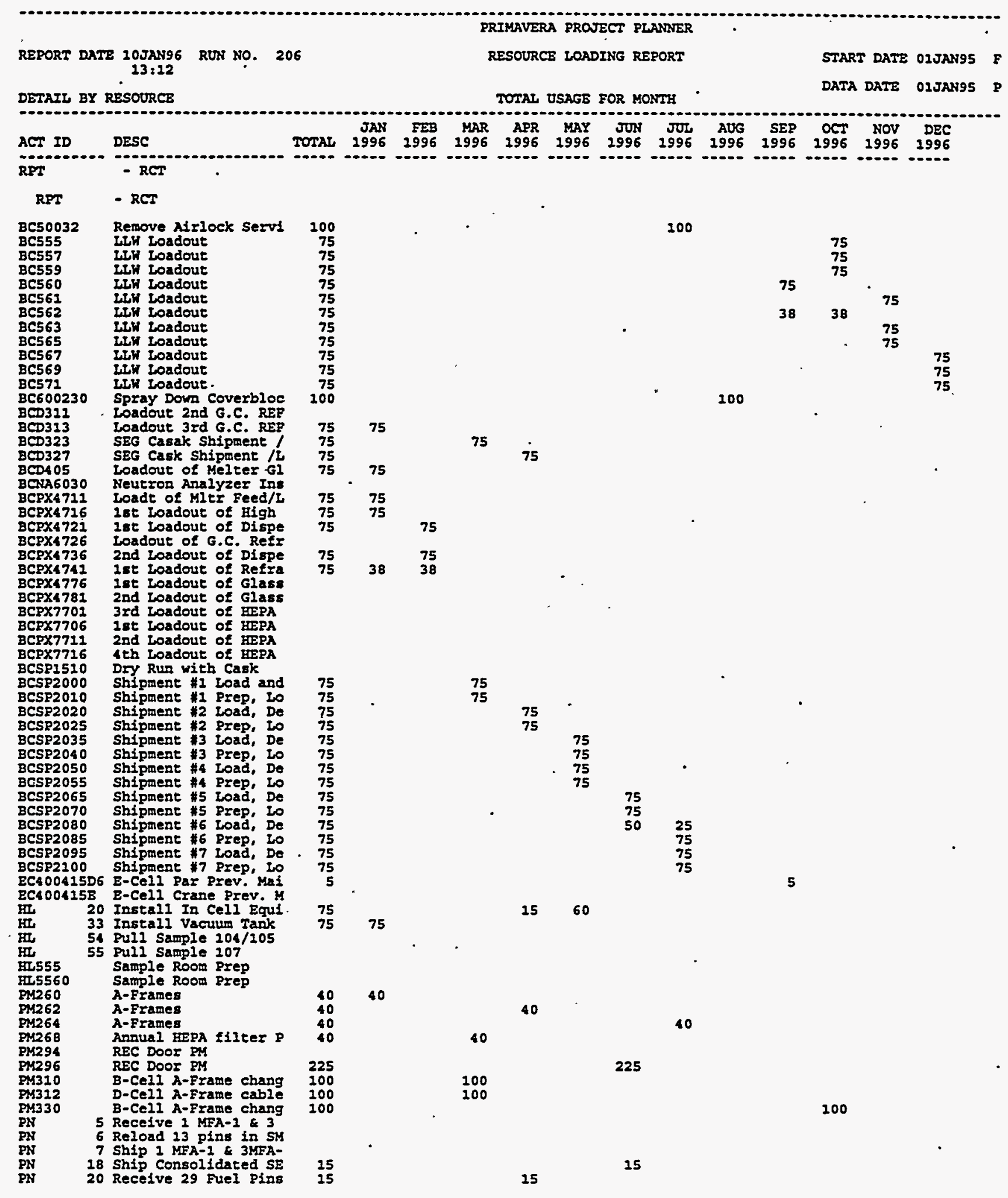


PRIMAVERA PROTECT PLANNER

REPORT DATE, $10 J A N 96$ RUN NO. $206 \cdot$ RESOURCE LOADING REPORT START DATE OIJAN95 E $13: 12$

DATA DATE 01JAN95

DETATL BY RESOURCB

TOSAZ USAGE FOR MONTH

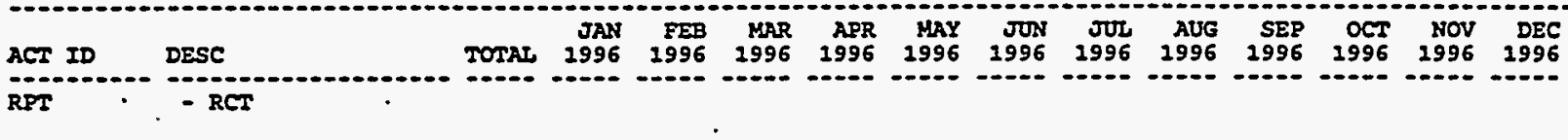

21 Transfer 9 ACO-3 Pin 23 store C-I Shipment 24 Ship C-I shipment to 25 Ship MFA-1/MFA-2/ACO 26 Ship PNC Waste (SERP 27 Recelve MFA-2 DS at

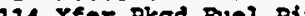
115 115 Tra 137 Ship Capoules to 324 S-Cell Par Prev. Mai
S-Cell Crane Prev. M Waste Disposal Waste Disporal Waste Disposal Waste Disposal Taste Disposal Nasee Disposal Naste Disposal Waste Disposal faste Disposal taste Disposa aste Disposal

SMP AI Crane Prev. $M$ Misc. Cask Load/Unlo Misc. Cask Load/Unio uise. Cask Load/Unio use. Cask toad/Unio Mse. Cask Load/0nlo Mise. Cask Load/Unlo Misc. Cask Load/Onlo Mise. Cask Load/Unlo Hisc. Cask Load/Unlo Misc. Cask Load/Unlo Mise. Cask Load/Onlo Mise. Cask Ioad/Onlo Mise. Cask Ioad/0nlo Mis. Cask Ioad/unlo Me. Cask Ioad/Unio Misc. Cask Load/Unlo Misc. Cask Load/Unlo Misc. Cask Load/Ualo Misc. Cask Load/Onlo Misc. Cask Load/Onlo Mise. Cask Load/Unlo Misc. Cask Iond/Unlo Hise. Cack toad/Unio His. Cask toadyolo 15e. Cask Load/usi Mise. Cask Load/Onlo Misc. Cask Load/Onlo Hisc. Cask Load/Unl Misc. Cask Load/unlo Mise. Cask Load/Unlo Mise. Cask Load/Jnlo Misc. Cask toad/Onlo Misc. Cask Toad/Jnlo Mis. Cask Loadunio Misc. Cask Ioad/Talo Misc. Cask Ioad/unlo Misc. Cask Load/unl Misc. Cask Load/Unlo MisC. Cask Load/Unl Mic. Cask Ioad/Unlo Misc. Cask Ioad/Unio Misc. Cask Ioad/Unlo Misc. Cask Ioad/Unio Misc. Cask Ioad/Unlo Misc. Cask Iord/Unlo Misc. Cask Load/Uno Misc. Cask Load/Unio Misc. Cask Load/Onlo
Misc. Cask Load/Unlo Misc. Cask Load/Unio Misc. Cask Ioad/UnIo
10

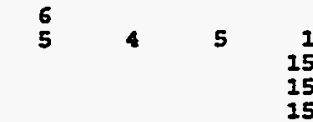

25

50

10

10

10

10

10 $\mathbf{5}$
$\mathbf{5}$
$\mathbf{5}$
$\mathbf{5}$
$\mathbf{5}$

5
5
5
5

5
5
5
5

5
5
5
5

5
5
5
5 


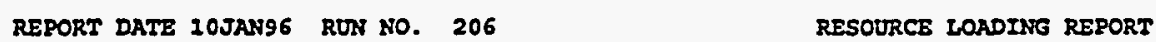

DETAIL BY RESOURCE TOTAL USAGE FOR MONHA

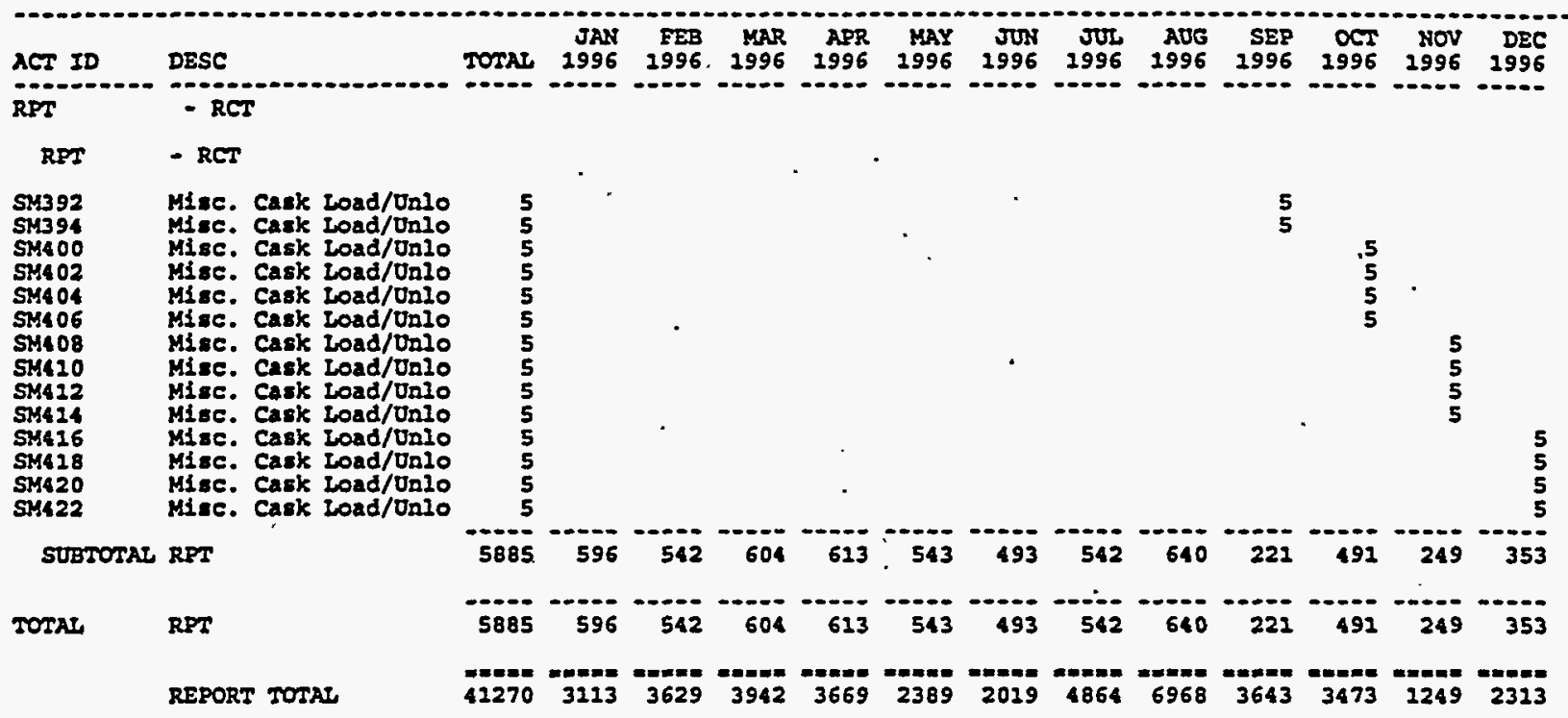




\title{
RESOURCE
}

\section{LOADING}

\author{
DETAIL
}

BY RESOURCE

$$
\text { KEH }
$$




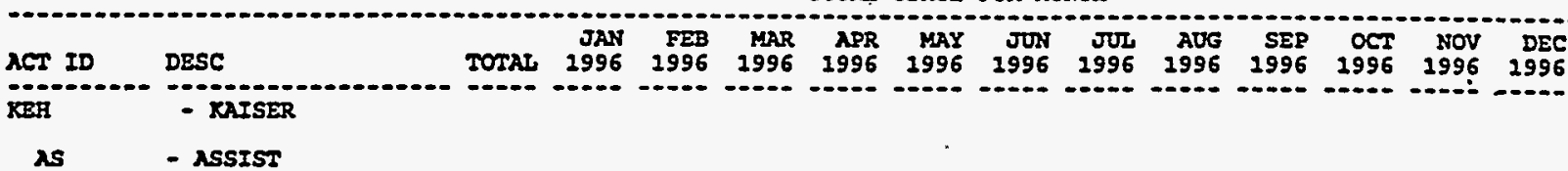

BC 250055 Remove 6-Ton Crane

BC 250060 Instai1 10-Ton Capac

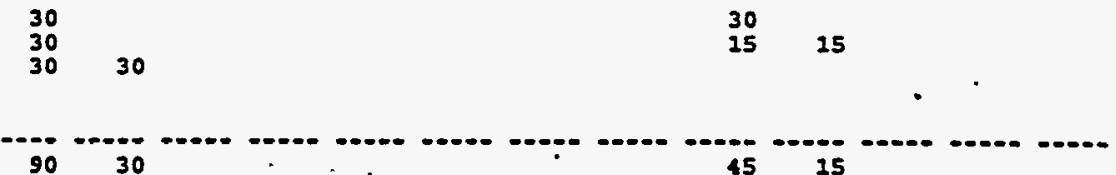

BCI1120 INSTALI 3-3/2 TON CR

BCHCB015 Core Dril1 Airlock BCHC8020 Install Compactor in

SUBTOTAT AS

$90 \quad 30$

$45 \quad 15$

EI

- ELECTRICIAN

BC 150055 Remove 6-Ton Crane

BC 150060 Install 10-Ton Capac

BC14120 INSTALI $3-1 / 2$ TON CR
BCWCB 020 Install Compactor in

SUBTOTAT ET

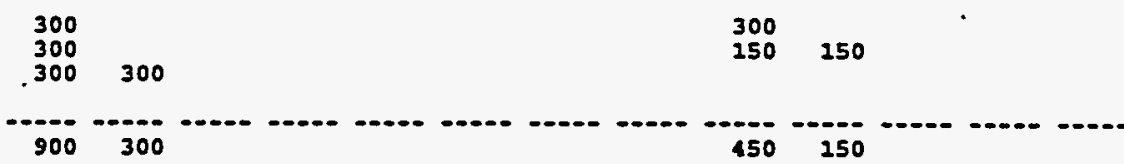

IR

- IRONWORKER

BC 150055 Remove 6-Ton Crane BC 150060 Install 10-Ton Capac BC21120 INSTALL 3-1/2 TON $C R$ SUBTOTAT IR

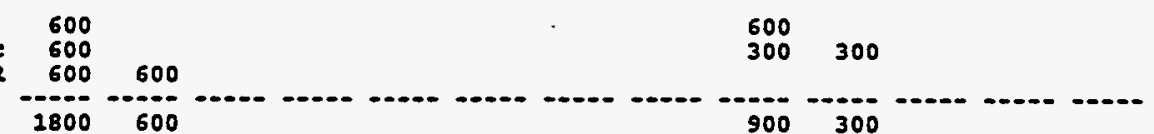

\section{IA - IABORER}

\begin{tabular}{|c|c|}
\hline $\begin{array}{l}3 C 150055 \\
3 C 150060 \\
3 C 21120 \\
3 C 180 \\
3 C 182 \\
3 C 184 \\
3 C 186 \\
3 C 188 \\
3 C 190 \\
3 C 192 \\
3 C 194 \\
3 C 196 \\
3 C 198 \\
3 C 200 \\
3 C 202 \\
3 C 298 \\
3 C 300 \\
3 C 302 \\
3 C A C 8015 \\
3 C H C 8020\end{array}$ & $\begin{array}{l}\text { Remove 6-Ton Crane } \\
\text { InstaIl } 10-T o n \text { Capac } \\
\text { INSTAL } 3-1 / 2 \text { ToN CR } \\
\text { Waste Compaction/Dec } \\
\text { Waste Compaction/DeC } \\
\text { Hante Compaction/Dec } \\
\text { Warte Compaction/Dec } \\
\text { Waste Compaction/Dec } \\
\text { Waste Compaction/Dec } \\
\text { Waste Compaction/Dec } \\
\text { Waste Compaction/Dec } \\
\text { Waste Compaction/Dec } \\
\text { Waste Compaction/Dec } \\
\text { Waste Compaction/Dec } \\
\text { Haste Compaction/Dec } \\
\text { Waste Compaction/Dec } \\
\text { Waste Compaction/Dec } \\
\text { Wante Compaction/Dec } \\
\text { Core Drili Airiock W } \\
\text { Install Compactor in }\end{array}$ \\
\hline
\end{tabular}

SUBTOTAL IA

PF - PIPEEITTER

BCAC8015 Core Drill Afrlock SUBTOTAL PF
UND
- UNDRESSER

BC 150055 Remove 6-Ton Crane BC 250060 Install 10-Ton Capac BC14120 INSTAIT 3-2/2 TON CR BCHCB015 Core Drill Airlock BChCB020 Install Compactor in SUBTOTAL OND
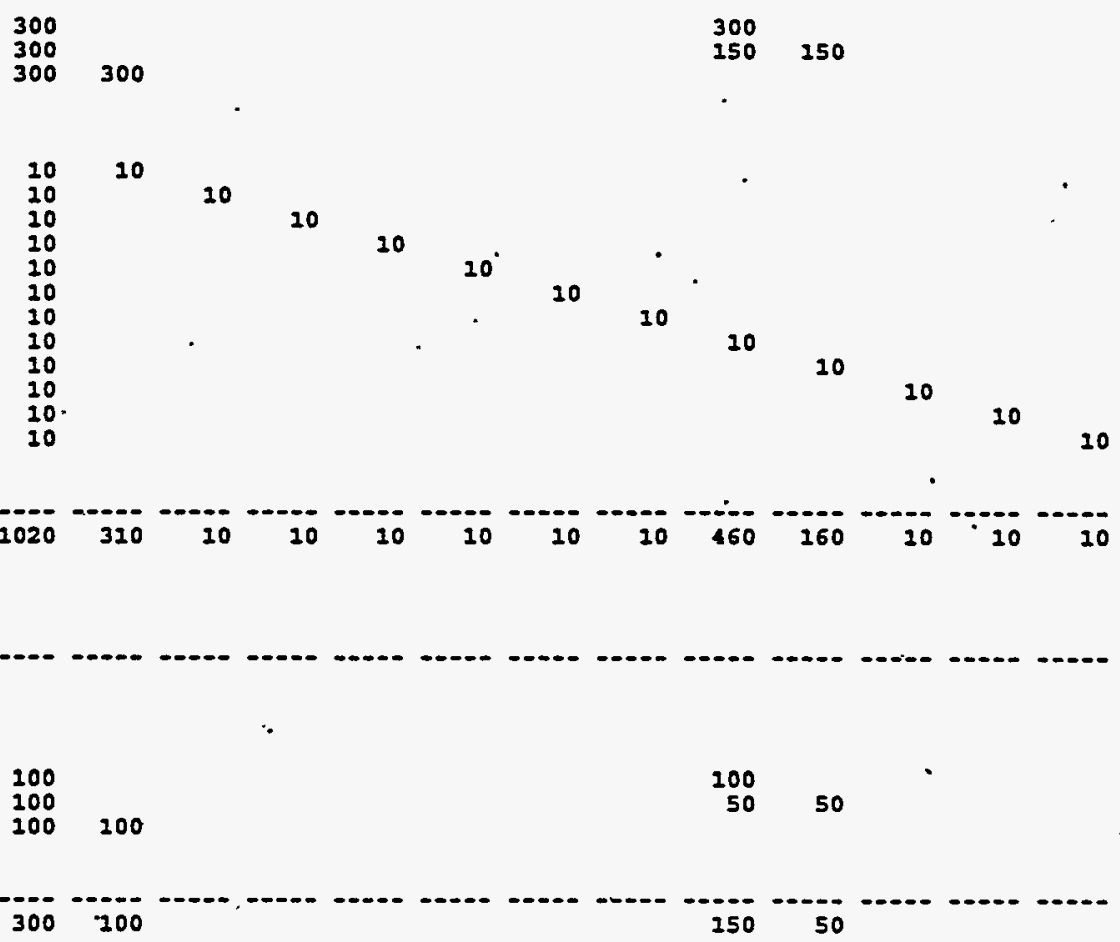


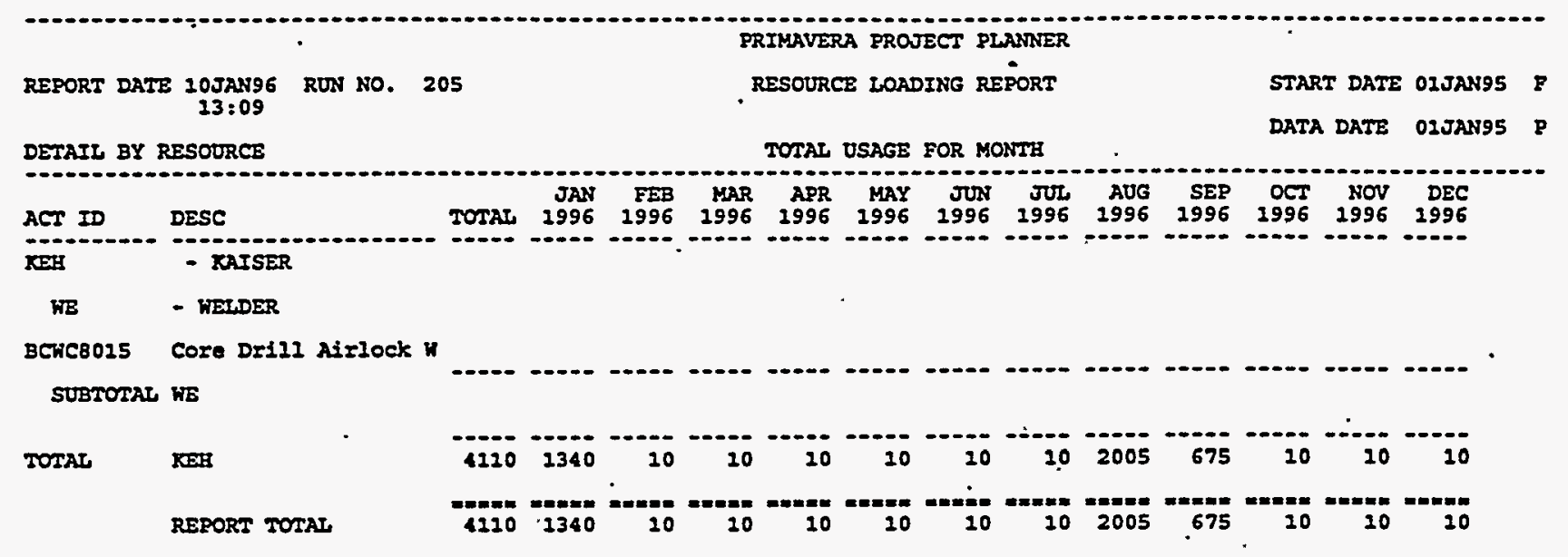


PRIMAVERA PROJECT PLARDIER

REPORT DATE 085AN96 RUN NO. 200 RESOURCE LOADING REPORT

START DATE O1JAN95 F

RESOURCE LOADING - TOTAL MONTFILY SUMAARY

TOTAL USAGE FOR MONTH

DATA DATE O1JAN95 $P$

\begin{tabular}{|c|c|c|c|c|c|c|c|c|c|c|c|c|c|c|}
\hline ACT ID & DESC & TOTAL & $\begin{array}{r}\text { JAN } \\
2996\end{array}$ & $\begin{array}{r}\text { EEB } \\
1996\end{array}$ & $\begin{array}{r}\text { MAR } \\
1996\end{array}$ & $\begin{array}{r}\text { APR } \\
1996\end{array}$ & $\begin{array}{r}\text { MAY } \\
1996\end{array}$ & $\begin{array}{r}\text { JUN } \\
.1996\end{array}$ & $\begin{array}{r}\pi \pi \\
1996\end{array}$ & $\begin{array}{r}\text { AUG } \\
1996\end{array}$ & $\begin{array}{r}\text { SEP } \\
1996\end{array}$ & $\begin{array}{r}\text { OCT } \\
1996\end{array}$ & $\begin{array}{r}\text { Nov } \\
2996\end{array}$ & $\begin{array}{r}\text { DEC } \\
1996\end{array}$ \\
\hline CRAFT & - CRAFT & & & & & & & & & & & & & \\
\hline $\begin{array}{l}\mathrm{AB} \\
\mathrm{EH}\end{array}$ & $\begin{array}{l}\text { AIR BALANCE } \\
\text { ELECTRICIAN } \\
\text { HILURIGET }\end{array}$ & $\begin{array}{r}1760 \\
3290 \\
12360\end{array}$ & $\begin{array}{l}240 \\
465\end{array}$ & $\begin{array}{r}600 \\
1335\end{array}$ & $\begin{array}{r}440 \\
60 \\
975\end{array}$ & $\begin{array}{r}40 \\
540 \\
1145\end{array}$ & 395 & 455 & $\begin{array}{r}240 \\
650 \\
1675\end{array}$ & $\begin{array}{r}200 \\
1000 \\
3185\end{array}$ & $\begin{array}{r}440 \\
1345\end{array}$ & $\begin{array}{l}400 \\
865\end{array}$ & 55 & 200 \\
\hline TOTAL & CRAFT & 17410 & 705 & 1935 & 2475 & 1725 & 395 & 455 & 2565 & 4385 & 2785 & 1265 & 55 & 665 \\
\hline
\end{tabular}

HCO - HOT CELL OPERATIONS

$\begin{array}{ll}\text { AS } & \text { ASSIST } \\ \text { ENY } & \text { ENIRY PERSON } \\ \text { LA } & \text { LABORER }\end{array}$

TECR HOT CELL TSCHNICIAN

TOTAL HCO

KER - KAJSER

AS ASSTST

EI ELECTRICIAN

IR - IRONWORKE

PF TABORER

UND UNDRESSER

HE HELDER

TOTAL XEH

RPT - RCT

RPT RCT

TOTAL RPT

REPORT TOTAL

\begin{tabular}{rrrrrrrrrrrrr}
1500 & 153 & 93 & 143 & 106 & 136 & 97 & 348 & 80 & 48 & 128 & 75 & 95 \\
5450 & 775 & 375 & 600 & 470 & 680 & 100 & 500 & & 225 & 525 & 450 & 450 \\
1200 & 100 & 100 & 100 & 100 & 100 & 100 & 100 & 100 & 100 & 100 & 100 & 100 \\
7620 & 620 & 260 & 875 & 334 & 341 & 319 & 659 & 1403 & 1160 & 860 & 230 & 560 \\
2205 & 165 & 325 & 145 & 321 & 194 & 155 & 150 & 360 & 105 & 105 & 90 & 90 \\
\hline 17975 & 1813 & 1153 & 1863 & 1331 & 1451 & 1070 & 1757 & 1943 & 1638 & 1718 & 945 & 1295
\end{tabular}

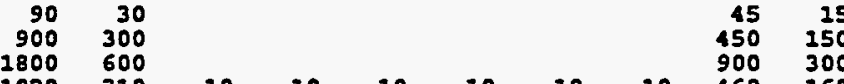

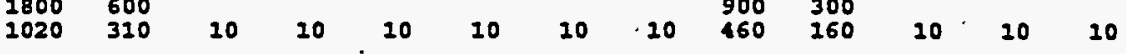

$300 \quad 100 \quad 150 \quad 50$

41101340

$\begin{array}{lllllllllllll}5885 & 596 & 542 & 604 & 6,3 & 543 & 493 & 542 & 640 & 221 & 491 & 249 & 353\end{array}$

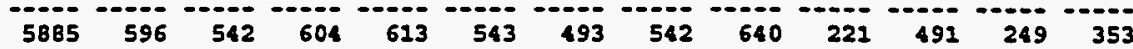

45380 - 


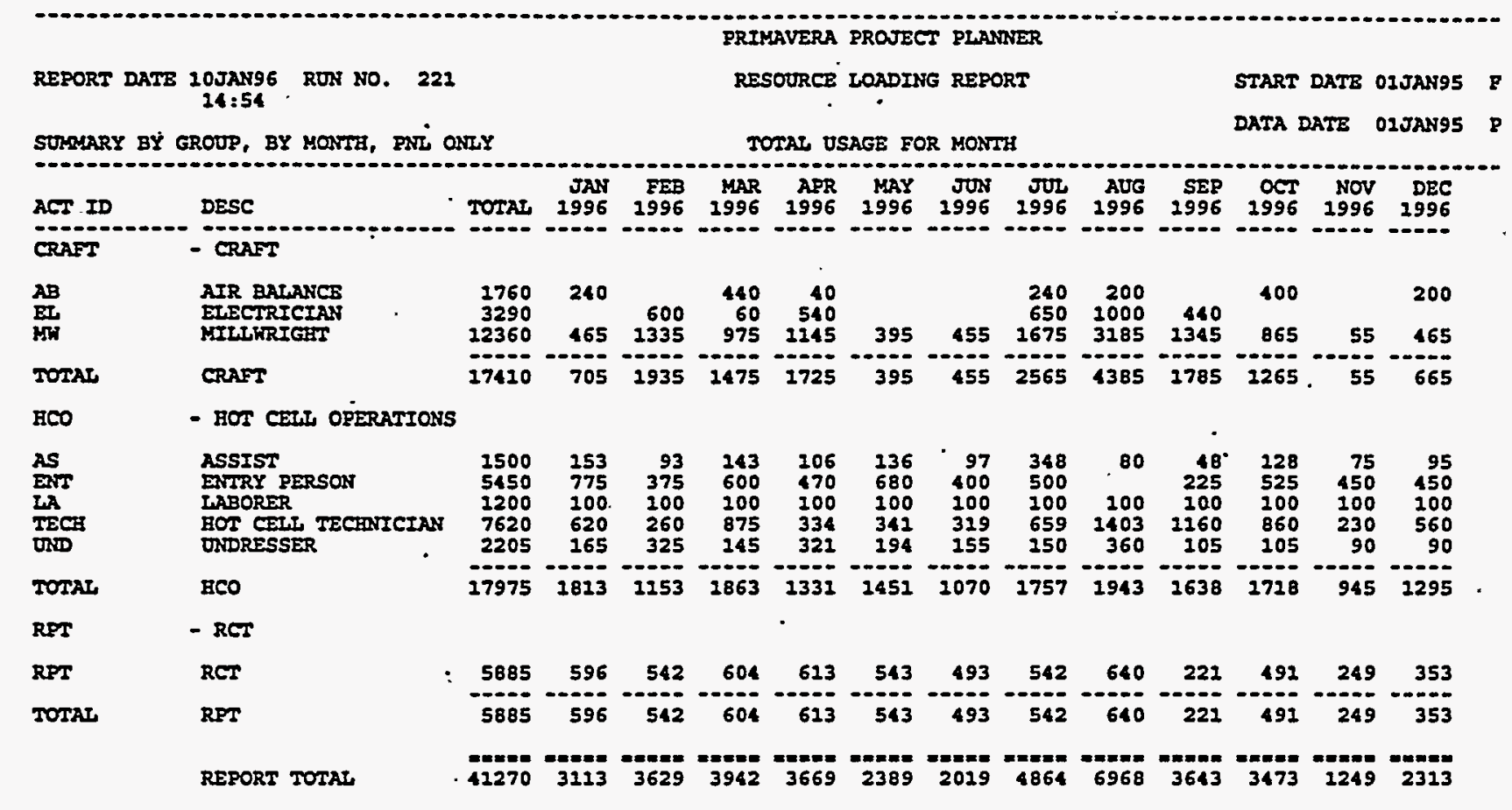




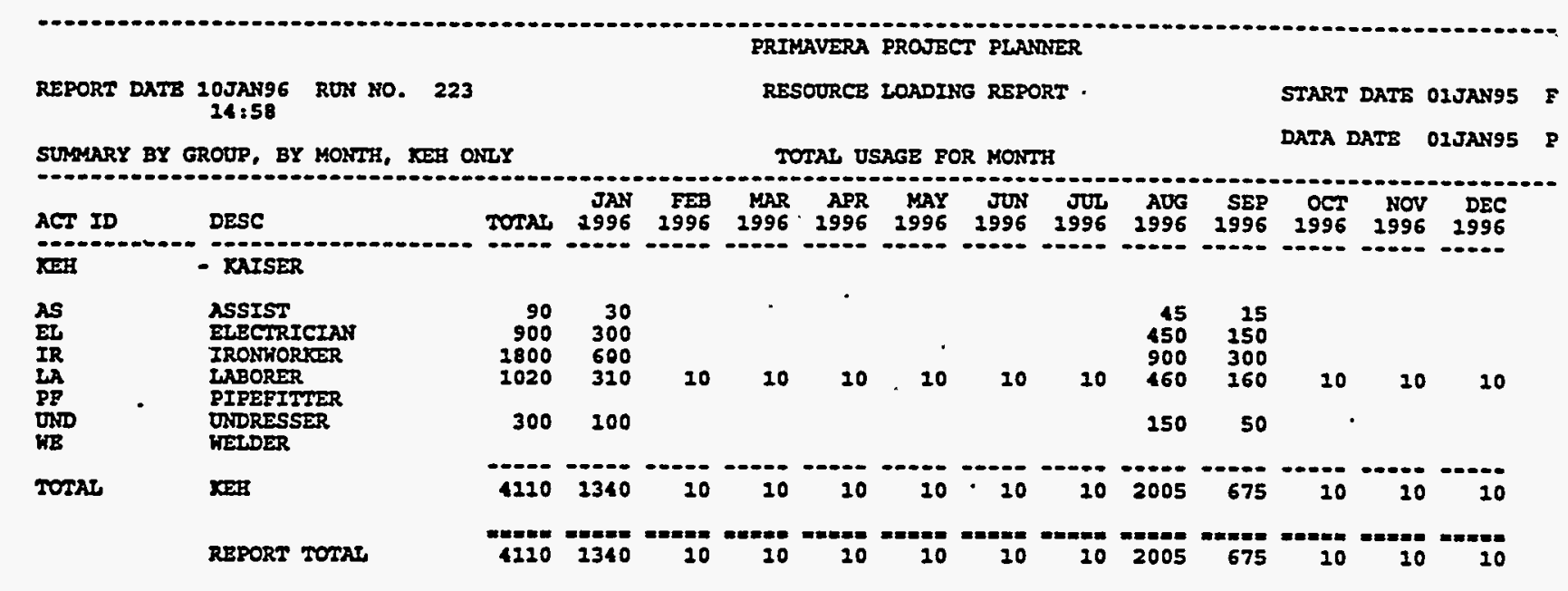




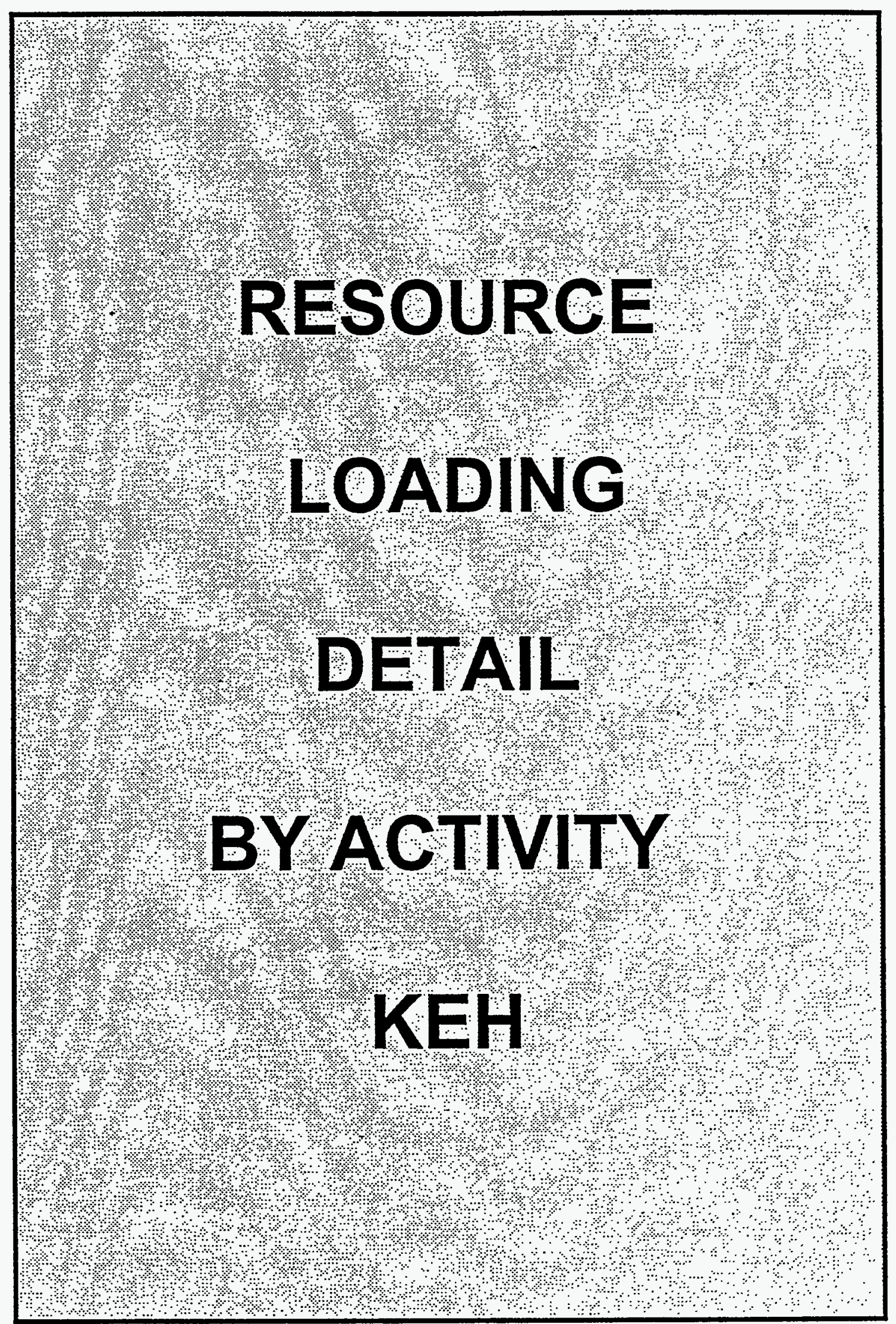


PRIMAVERA PROJECT PLAMLNER

RESOURCE LOADING DETAIL BY ACTIVITY - KEH

JAH FEB MAR APR MAY JU JU AUG SEP OCT NOV DEC

RESOURCE RESOURCE DESCRIPTION TOTAT 1996

- 250055 - Remove 6-Ton Crane

$\begin{array}{ll}\text { AS } & \text { ASSIST } \\ \text { EI } & \text { ELECIRICIAN } \\ \text { IR } & \text { IRONHORKER } \\ \text { IA } & \text { LABORER } \\ \text { UND } & \text { UNDRESSER }\end{array}$

TorAL BC 250055

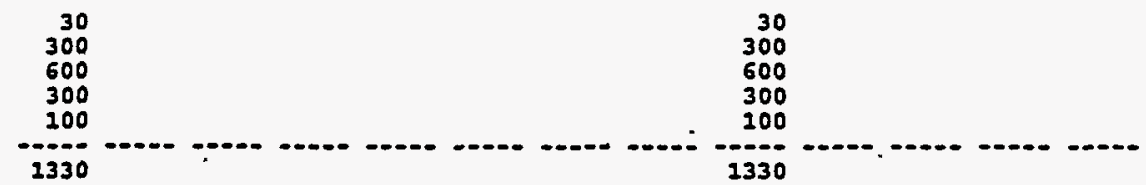

BC 250060 - Instal 10-Ton Capacity Crane

\begin{tabular}{|c|c|c|c|c|}
\hline $\begin{array}{l}\text { AS } \\
\text { II } \\
\text { IR } \\
\text { UR } \\
\text { UND }\end{array}$ & $\begin{array}{l}\text { ASSIST } \\
\text { ELECIRICIAN } \\
\text { IRONKORRER } \\
\text { IABORER } \\
\text { UNDRESSER }\end{array}$ & $\begin{array}{l}30 \\
300 \\
600 \\
300 \\
100\end{array}$ & $\begin{array}{r}15 \\
150 \\
300 \\
150 \\
50\end{array}$ & $\begin{array}{r}15 \\
150 \\
300 \\
250 \\
50\end{array}$ \\
\hline
\end{tabular}

TOTAL BC -250060

gC14220 - INSTAII 3-2/2 TON CRANB

$\begin{array}{llrr}\text { IS } & \text { ASSIST } & 30 & 30 \\ \text { ER } & \text { ELECTRICIAR } & 300 & 300 \\ \text { IR } & \text { IRONWORKER } & 600 & 600 \\ \text { IR } & \text { LRBORER } & 300 & 300 \\ \text { UND } & \text { UNDRESSER } & 100 & 100 \\ \text { TOTAI } & \text { BC14120 } & 1330 & 1330\end{array}$

BC1E6 - Waste Compaction/Decon

UA LABORER

TOTAL BC186

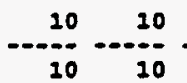

BC188 - Waste Compaction/Decon

w1 TABORER

TOTAL BC288

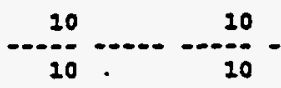

BC190 - Waste Compaction/Decon

Torar $\quad$ ac190

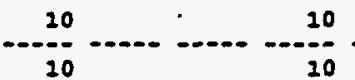

BC192 - Waste Compaction/Decon

IA IABORER.

TOTAL BC192

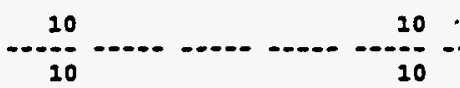

BC294 - Haste Compaction/Decon

In LABORER

TOTAL BC194

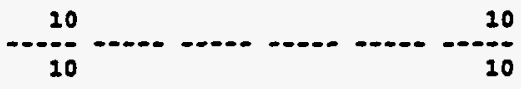

BC196 - Waste Compaction/Decon

I.

TOTAT LABORER

20

10

8C198 - Haste compaction/Decon

LA LABORER

TOTAL BC298

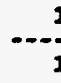

10 10 
TOTAL USAGE FOR MONTH

DATA DATE 01JAN9S PAGE No. 2

RESOURCE LOADIHG DETAII BY ACTIVITY - KDR

IOTAT USAGE POR MONTH

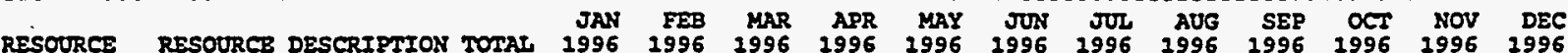

200 - Haste Compaction/Decon

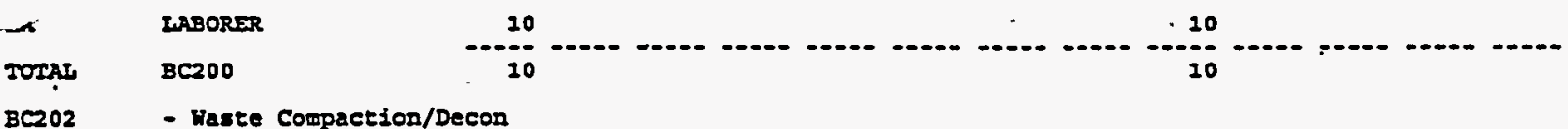

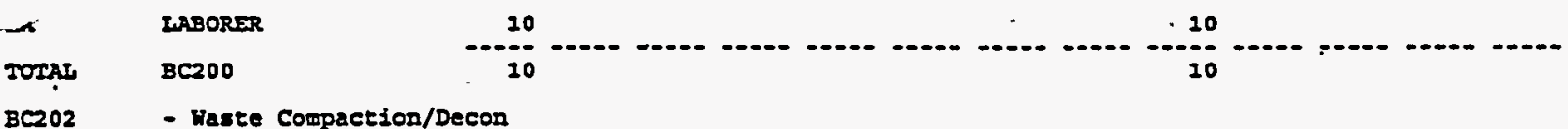

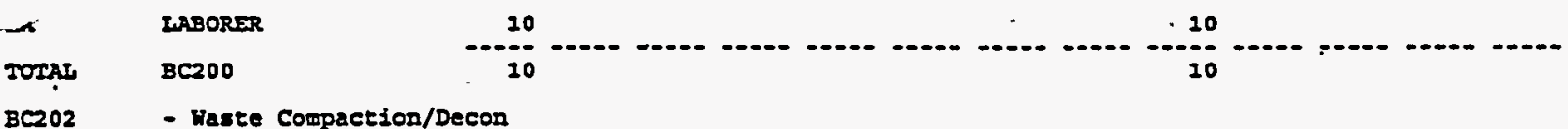

IA IABORER 10

$\mathrm{BC2O2} \because 10$

10

10

BC298 - Waste Compaction/Decon

IAR . IABORER

TOTAI BC298

$-10$

BC300

IA

- Waste Compaction/Decon

LABORER

TOTAI " BC300

10

$-\frac{10}{10}$

10

BC302 - Naste Compaction/Decon

IA

TABORER

10

$\frac{20}{20}$

TOTAT BC302

REPORT TOTAL

10

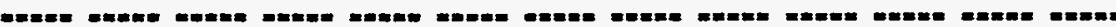

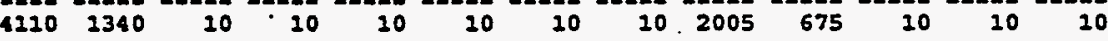




\section{Distribution}

No. Of

Copies

Offsite

2 Office of Scientific and Technical Information

Onsite

3 DOE Richland Operations Office

J.E. Trevino

C. R. Richins

D. C. Langstaff
No. Of

Copies

Onsite

24 Pacific Northwest National Laboratory

M. J. Bagaglio, Jr.

W. B. Chrisler

D. P. Higby

J. D. Jacobsen

S. L. Keller

D.E. Knowlton

J. K. Larsen

S. D. Landsman (5)

C. A. Peterson

K. H. Pryor

C. N. Swanson

R. E. Thomhill (3)

P. J. Weaver

Technical Report Files (5)

Dist. 1 
